\title{
Homological finiteness of Abelian covers
}

\author{
AleXander I. Suciu, YAPING YANG AND Gufang ZhaO
}

\begin{abstract}
We present a method for deciding when a regular Abelian cover of a finite CW-complex has finite Betti numbers. To start with, we describe a natural parameter space for all regular covers of a finite $\mathrm{CW}$-complex $X$, with group of deck transformations a fixed Abelian group $A$, which in the case of free Abelian covers of rank $r$ coincides with the Grassmanian of $r$-planes in $H^{1}(X, \mathbb{Q})$. Inside this parameter space, there is a subset $\Omega_{A}^{i}(X)$ consisting of all the covers with finite Betti numbers up to degree $i$.

Building up on work of Dwyer and Fried, we show how to compute these sets in terms of the jump loci for homology with coefficients in rank-1 local systems on $X$. For certain spaces, such as smooth, quasi-projective varieties, the generalized Dwyer-Fried invariants that we introduce here can be computed in terms of intersections of algebraic subtori in the character group. For many spaces of interest, the homological finiteness of Abelian covers can be tested through the corresponding free Abelian covers. Yet in general, Abelian covers exhibit different homological finiteness properties than their free Abelian counterparts.
\end{abstract}

Mathematics Subject Classification (2010): 14F35 (primary); 55N25, 20J05, 57M07 (secondary).

\section{Introduction}

By classical covering space theory, the connected, regular covers of a CW-complex are classified by the quotients of its fundamental group. In this paper, we investigate the set of covers with fixed deck-transformation group (usually taken to be an Abelian group) for which the Betti numbers up to a fixed degree are finite.

\subsection{A parameter set for regular covers}

Let $X$ be a connected CW-complex with finite 1-skeleton. Let $G=\pi_{1}\left(X, x_{0}\right)$ be the fundamental group, and let $A$ be a quotient of $G$. The regular covers of $X$ with

The first author was partially supported by NSA grant H98230-09-1-0021 and NSF grant DMS1010298.

Received May 28, 2012; accepted November 28, 2012. 
group of deck transformations isomorphic to $A$ can be parametrized by the set

$$
\Gamma(G, A)=\operatorname{Epi}(G, A) / \operatorname{Aut}(A),
$$

where $\operatorname{Epi}(G, A)$ is the set of all epimorphisms from $G$ to $A$ and $\operatorname{Aut}(A)$ is the group of automorphisms of $A$, acting on $\operatorname{Epi}(G, A)$ by composition. For an epimorphism $\nu: G \rightarrow A$, we write its class in $\Gamma(G, A)$ by $[v]$, and the corresponding cover by $X^{v} \rightarrow X$

In the case when $A$ is Abelian, the parameter set for $A$-covers may be identified with $\Gamma(H, A)$, where $H=H_{1}(X, \mathbb{Z})$. Our first result identifies this set with a (settheoretical) twisted product over a rational Grassmannian. More precisely, let $\bar{H}$ be the maximal, torsion-free Abelian quotient of $H$, and identify $\bar{H}=\mathbb{Z}^{n}$ and $\bar{A}=\mathbb{Z}^{r}$. By linear algebra (see, e.g., [2, Section 12, Theorem 4.3]), any rational subspace of $\mathbb{Z}^{n} \otimes \mathbb{Q}$ has a primitive sublattice, so this allows us to identify $\operatorname{Gr}_{r}\left(\mathbb{Z}^{n}\right)$ with $\mathrm{Gr}_{r}\left(\mathbb{Q}^{n}\right)$. Let $\mathrm{P}$ be a parabolic subgroup of $\mathrm{GL}_{n}(\mathbb{Z})$, such that $\mathrm{GL}_{n}(\mathbb{Z}) / \mathrm{P}$ is the Grassmannian $\mathrm{Gr}_{n-r}\left(\mathbb{Z}^{n}\right)$.

Theorem A (Theorem 3.2). There is a bijection

$$
\Gamma(H, A) \longleftrightarrow \mathrm{GL}_{n}(\mathbb{Z}) \times_{\mathrm{P}} \Gamma,
$$

where $\Gamma$ is the finite set $\Gamma(H / \bar{A}, A / \bar{A})$, and $\mathrm{GL}_{n}(\mathbb{Z}) \times_{\mathrm{P}} \Gamma$ is the twisted product of $\mathrm{GL}_{n}(\mathbb{Z})$ and $\Gamma$ under the natural action of $\mathrm{P}$ on the two sets.

\subsection{Dwyer-Fried sets and their generalizations}

In a foundational paper on the subject, [6], Dwyer and Fried considered the regular covers of a finite, connected CW-complex $X$, with group of deck transformations $A=\mathbb{Z}^{r}$. Inside the parameter space $\Gamma\left(H, \mathbb{Z}^{r}\right)=\operatorname{Gr}_{r}\left(\mathbb{Q}^{n}\right)$, where $n=$ rank $H$, they isolated the sets $\Omega_{r}^{i}(X)$, consisting of those covers for which the Betti numbers up to degree $i$ are finite.

The Dwyer-Fried sets $\Omega_{r}^{i}(X)$ have since been studied in depth in $[13,14]$, using the characteristic varieties of $X$. These varieties, $V^{i}(X)$, are Zariski closed subsets of the character group $\widehat{H}=\operatorname{Hom}\left(H, \mathbb{C}^{*}\right)$; they consist of those rank-1 local systems on $X$ for which the corresponding cohomology groups do not vanish, for some degree less or equal to $i$.

We further develop this theory here, by first defining the generalized DwyerFried invariants of $X$ to be the subsets $\Omega_{A}^{i}(X)$ of $\Gamma(G, A)$ consisting of those regular $A$-covers having finite Betti numbers up to degree $i$. In the case when $A$ is a finitely generated (not necessarily torsion-free) Abelian group, we establish a similar formula, computing the invariants $\Omega_{A}^{i}(X)$, viewed now as subsets of $\Gamma(H, A)$, in terms of the characteristic varieties of $X$.

Theorem B (Theorem 10.5). Let $X$ be a connected, finite $C W$-complex, and let $H=H_{1}(X, \mathbb{Z})$. Suppose $v: H \rightarrow A$ is an epimorphism to an Abelian group $A$. Then

$$
\Omega_{A}^{i}(X)=\left\{[\nu] \in \Gamma(H, A) \mid \operatorname{im}(\hat{v}) \cap V^{i}(X) \text { is finite }\right\},
$$


where $V^{i}(X) \subset \widehat{H}$ is the $i$-th characteristic variety of $X$, and $\hat{v}: \widehat{A} \rightarrow \widehat{H}$ is the induced morphism between the character groups.

\subsection{An upper bound for the $\Omega$-sets}

In order to estimate the size of the Dwyer-Fried sets, it is convenient to look at various analogues of the incidence subvarieties of the Grassmannian, known as the special Schubert varieties.

Given a subgroup $\xi \leq H$ and an Abelian group $A$, let $\sigma_{A}(\xi)$ be the set of all $[v] \in \Gamma(H, A)$ for which $\operatorname{rank}(\operatorname{ker}(\nu)+\xi)<\operatorname{rank} H$, and let $U_{A}(\xi)$ be the subset of those $[v]$ for which, additionally, $\operatorname{ker}(v) \cap \bar{\xi} \subseteq \xi$, where $\bar{\xi}$ denotes the primitive closure of $\xi$.

Each subgroup $\xi \leq H$ gives rise to an algebraic subgroup, $V(\xi)=\widehat{H / \xi}$, of the character group $\widehat{H}$, with identity component $V(\bar{\xi})$. Given a subvariety $W \subset \widehat{H}$, and a positive integer $d$, let $\Xi_{d}(W)$ be the collection of all subgroups $\xi \leq H$ for which the determinant group $\bar{\xi} / \xi$ is cyclic of order dividing $d$, and there is a generator $\eta$ of $\widehat{\xi / \xi}$ such that $\eta V(\bar{\xi})$ is a maximal, positive-dimensional translated subtorus in $W$. (The set $\Xi_{1}(W)$ is essentially the same as the "exponential tangent cone" from $[4,14]$.)

Theorem $\mathbf{C}$ (Theorem 10.7). Let $H=H_{1}(X, \mathbb{Z})$, and let $A$ be a quotient of $H$. Then:

$$
\Omega_{A}^{i}(X) \subseteq \Gamma(H, A) \backslash \bigcup_{d \geq 1} \bigcup_{\xi \in \Xi_{d}\left(V^{i}(X)\right)} U_{A}(\xi) .
$$

In the case when $A$ has rank one, we show in Theorem 12.1 that this upper bound is reached; furthermore, we only need to use in this case the set $\Xi_{C(A)}\left(V^{i}(X)\right)$, where $c(A)$ is the largest order of any element in $A$. In other words, if $\operatorname{rank} A=1$, then

$$
\Omega_{A}^{i}(X)=\Gamma(H, A) \backslash \bigcup_{\xi \in \Xi_{c(A)}\left(V^{i}(X)\right)} U_{A}(\xi) .
$$

\subsection{Translated subgroups in the characteristic varieties}

For a large class of spaces - for instance, smooth, complex quasi-projective varieties - the characteristic varieties are union of translated algebraic subgroups of $\widehat{H}$. Using techniques from [15], we obtain several explicit formulas in this situation, expressing the Dwyer-Fried sets purely in terms of the corresponding subgroups of $H$, and the associated translation factors.

Theorem D (Theorem 13.1). Suppose $V^{i}(X)=\bigcup_{j=1}^{s} \eta_{j} V\left(\xi_{j}\right)$, where $\xi_{1}, \ldots, \xi_{s}$ are subgroups of $H=H_{1}(X, \mathbb{Z})$, and $\eta_{1}, \ldots, \eta_{s}$ are torsion elements in $\widehat{H}$. Then

$$
\Omega_{A}^{i}(X)=\Gamma(H, A) \backslash \bigcup_{\xi \in \Xi_{c}\left(V^{i}(X)\right)} U_{A}(\xi),
$$

where $c$ is the least common multiple of $\operatorname{ord}\left(\eta_{1}\right) \cdot c\left(\bar{\xi}_{1} / \xi_{1}\right), \ldots, \operatorname{ord}\left(\eta_{s}\right) \cdot c\left(\bar{\xi}_{s} / \xi_{s}\right)$. 
A formula of a different flavor is given in Theorem 13.2. A particular case of this formula is worth singling out: if $V^{i}(X)=V\left(\xi_{1}\right) \cup \cdots \cup V\left(\xi_{s}\right)$ is a union of algebraic subgroups of $\widehat{H}$, then

$$
\Omega_{A}^{i}(X)=\Gamma(H, A) \backslash \bigcup_{j=1}^{s} q^{-1}\left(\sigma_{\bar{A}}\left(\xi_{j}\right)\right),
$$

where $q$ is the canonical projection $\Gamma(H, A)$ onto the rational Grassmannian $\Gamma(H, \bar{A})$, and the sets $\sigma_{\bar{A}}\left(\xi_{j}\right)$ are usual special Schubert varieties.

\subsection{Abelian versus free Abelian covers}

This last formula brings up a rather general question: If a cover $X^{\bar{v}}$ has finite Betti numbers, does $X^{v}$ also have finite Betti numbers? This question can be answered by comparing the generalized Dwyer-Fried invariants of $X$ with their classical counterparts.

As before, let $A$ be a quotient of $H=H_{1}(X, \mathbb{Z})$, and set $r=\operatorname{rank} A$ and $n=\operatorname{rank} H$. The canonical projection $q: \Gamma(H, A) \rightarrow \Gamma(H, \bar{A})$ restricts to a map $\Omega_{A}^{i}(X) \rightarrow \Omega_{\bar{A}}^{i}(X)$ between the respective Dwyer-Fried sets, thus yielding the commuting diagram

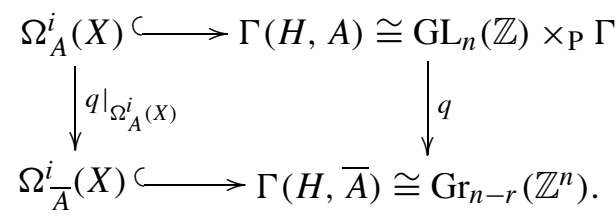

If $q^{-1}\left(\Omega_{\bar{A}}^{i}(X)\right)=\Omega_{A}^{i}(X)$, then the finiteness of the Betti numbers of an $A$-cover can be tested through the corresponding $\bar{A}$-cover. In general, though, diagram (1.4) is not a pullback diagram; in that case, the generalized Dwyer-Fried invariants, when viewed as homotopy type invariants, contain more information than the classical ones. This dichotomy is illustrated by the following result:

Theorem E (Propositions 13.6 and 13.9). Suppose the characteristic variety $V^{i}(X)$ is of the form $\bigcup_{j} \rho_{j} T_{j}$, where each $T_{j} \subset \widehat{H}$ is an algebraic subgroup, and each $\bar{\rho}_{j} \in \widehat{H} / T_{j}$ has finite order.

(i) If $\operatorname{ord}\left(\bar{\rho}_{j}\right)$ is coprime to the order of Tors $(A)$, for each $j$, then $q^{-1}\left(\Omega_{\bar{A}}^{i}(X)\right)=$ $\Omega_{A}^{i}(X)$.

(ii) If the identity component of $T_{1}$ is not contained in $\prod_{j \neq 1} T_{j}$, the order of $\rho_{1}$ divides $c(A)$, and $\operatorname{rank} A<\operatorname{rank} H-\operatorname{dim} \prod_{j \neq 1} T_{j}$, then $q^{-1}\left(\Omega_{A}^{i}(X)\right) \supsetneqq \Omega_{A}^{i}(X)$.

In other words, suppose $X^{\bar{\nu}}$ is a regular $\bar{A}$-cover of $X$, with finite Betti numbers up to degree $i$. Then, if (i) holds, all $A$-covers $X^{v}$ have the same finiteness property, whereas if (ii) holds, one of those $A$-covers will have an infinite Betti number in some degree less or equal to $i$. 


\subsection{Toric complexes}

A nice class of spaces to which our theory applies is that of toric complexes. Every simplicial complex $L$ on $n$ vertices determines a subcomplex $T_{L}$ of the $n$-torus, with fundamental group the right-angled Artin group associated to the 1-skeleton of $L$. Identify the group $H=H_{1}\left(T_{L}, \mathbb{Z}\right)$ with $\mathbb{Z}^{n}$. Work of Papadima and Suciu [12] shows that the characteristic varieties $V^{i}\left(T_{L}\right)$ are unions of coordinate subspaces in $\widehat{H}=\left(\mathbb{C}^{*}\right)^{n}$; equations for these subspaces can be read off directly from the simplicial complex $L$.

Formula (1.3) computes all the generalized Dwyer-Fried sets of a toric complex. More precisely, if $A$ is a quotient of $H$, then, as noted in Corollary 13.4, the complement of $\Omega_{A}^{i}\left(T_{L}\right)$ in $\Gamma(H, A)$ fibers over the special Schubert varieties associated to the coordinate subspaces comprising $V^{i}\left(T_{L}\right)$, with each fiber isomorphic to $\Gamma(H / \bar{A}, A / \bar{A})$. Thus, if $T_{L}^{\bar{v}}$ is a free Abelian cover of $T_{L}$, with finite Betti numbers up to some degree $i$, then all finite Abelian covers $T_{L}^{v} \rightarrow T_{L}^{\bar{v}}$ have the same homological finiteness property.

\subsection{Quasi-projective varieties}

Another important class of spaces to which our methods apply quite well is that of smooth, quasi-projective varieties. For such a space $X$, work of Arapura [1] and others shows that

$$
V^{1}(X)=Z \cup \bigcup_{\xi \in \Lambda} V(\xi) \cup \bigcup_{\xi \in \Lambda^{\prime}}(V(\xi) \backslash V(\bar{\xi})),
$$

where $Z$ is a finite set, and $\Lambda$ and $\Lambda^{\prime}$ are certain (finite) collections of subgroups of $H=H_{1}(X, \mathbb{Z})$.

Theorem $\mathbf{F}$ (Theorem 14.3). With notation as above, let $A$ be a quotient of $H$. Then

$$
\Omega_{A}^{1}(X)=\Gamma(H, A) \backslash\left(\bigcup_{\xi \in \Lambda} q^{-1}\left(\sigma_{\bar{A}}(\bar{\xi})\right) \cup \bigcup_{\xi \in \Lambda^{\prime}}\left(q^{-1}\left(\sigma_{\bar{A}}(\bar{\xi})\right) \cap \theta_{A}(\xi)\right)\right),
$$

where $\sigma_{\bar{A}}(\bar{\xi}) \subseteq \operatorname{Gr}_{n-r}\left(\mathbb{Z}^{n}\right) \cong \Gamma(\bar{H}, \bar{A})$ is a special Schubert variety, and $\theta_{A}(\xi)$ consists of those $[v] \in \Gamma(H, A)$ for which there is a subgroup $\xi \leq \xi^{\prime} \lesseqgtr \bar{\xi}$ such that $\bar{\xi} / \xi^{\prime}$ is cyclic and $\nu(x) \neq 0$ for all $x \in \bar{\xi} \backslash \xi^{\prime}$.

Theorem E, part (i) now shows the following: if the order of $\bar{\xi} / \xi$ is coprime to $c(A)$, for each $\xi \in \Lambda^{\prime}$, then $\Omega_{A}^{1}(X)=q^{-1}\left(\Omega_{\bar{A}}^{1}(X)\right)$.

In general, though, such an equality does not hold. Examples of this sort can be constructed using quasi-homogeneous surfaces (with the singularity at the origin removed), which are homotopy equivalent to Brieskorn manifolds $M=$ $\Sigma\left(a_{1}, \ldots, a_{n}\right)$. For instance, if $M=\Sigma(2,4,8)$, then the universal free Abelian cover of $M$ has finite $b_{1}$, whereas the universal Abelian cover of $M$ has infinite $b_{1}$. 


\subsection{Organization of the paper}

This paper is organized as follows: In Section 2 and Section 3, we describe the structure of the parameter set $\Gamma(H, A)$ for regular $A$-covers of a finite, connected CW-complex $X$ with $H_{1}(X, \mathbb{Z})=H$, while in Section 4 we define the generalized Dwyer-Fried invariants $\Omega_{A}^{i}(X)$, and study their basic properties.

In Section 5, we review the Pontryagin correspondence between subgroups of $H$ and algebraic subgroups of the character group $\widehat{H}$, while in Section 6 we associate to each subvariety $W \subset \widehat{H}$ a family of subgroups of $H$ generalizing the exponential tangent cone construction. In Section 7 and Section 8 , we introduce and study several subsets of the parameter set $\Gamma(H, A)$, which may be viewed as analogues of the special Schubert varieties and the incidence varieties from classical algebraic geometry.

In Section 9 we revisit the Dwyer-Fried theory in the more general context of (not necessarily torsion-free) Abelian covers, while in Section 10 we show how to determine the sets $\Omega_{A}^{i}(X)$ in terms of the jump loci for homology in rank-1 local systems on $X$. In Section 11, we compare the Dwyer-Fried invariants $\Omega_{A}^{i}(X)$ with their classical counterparts, $\Omega_{\bar{A}}^{i}(X)$, while in Section 12 we discuss in more detail these invariants in the case when $\operatorname{rank} A=1$.

Finally, in Section 13 we study the situation when all irreducible components of the characteristic varieties of $X$ are (possibly translated) algebraic subgroups of the character group, while in Section 14 we consider the particular case when $X$ is a smooth, quasi-projective variety.

\section{A parameter set for regular Abelian covers}

We start by setting up a parameter set for regular covers of a CW-complex, with special emphasis on the case when the deck-transformation group is Abelian.

\subsection{Regular covers}

Let $X$ be a connected CW-complex with finite 1-skeleton. Without loss of generality, we may assume $X$ has a single 0 -cell, which we will take as our basepoint, call it $x_{0}$. Let $G=\pi_{1}\left(X, x_{0}\right)$ be the fundamental group. Since the space $X$ has only finitely many 1-cells, the group $G$ is finitely generated.

Consider an epimorphism $v: G \rightarrow A$ from $G$ to a (necessarily finitely generated) group $A$. Such an epimorphism gives rise to a regular cover of $X$, which we denote by $X^{v}$. Note that $X^{v}$ is also a connected CW-complex, the projection map $p: X^{v} \rightarrow X$ is cellular, and the group $A$ is the group of deck transformations of $X^{\nu}$.

Conversely, every (connected) regular cover $p:\left(Y, y_{0}\right) \rightarrow\left(X, x_{0}\right)$ with group of deck transformations $A$ defines a normal subgroup $p_{\sharp}\left(\pi_{1}\left(Y, y_{0}\right)\right) \triangleleft \pi_{1}\left(X, x_{0}\right)$, with quotient group $A$. Moreover, if $v: G \rightarrow A$ is the projection map onto the 
quotient, then the cover $Y$ is equivalent to $X^{v}$, that is, there is an $A$-equivariant homeomorphism $Y \cong X^{v}$.

Let $\operatorname{Epi}(G, A)$ be the set of all epimorphisms from $G$ to $A$, and let $\operatorname{Aut}(A)$ be the automorphism group of $A$. The following lemma is standard:

Lemma 2.1. Let $G=\pi_{1}\left(X, x_{0}\right)$, and let $A$ be a group. The set of equivalence classes of connected, regular A-covers of $X$ is in one-to-one correspondence with

$$
\Gamma(G, A):=\operatorname{Epi}(G, A) / \operatorname{Aut}(A)
$$

the set of equivalence classes [v] of epimorphisms $v: G \rightarrow A$, modulo the rightaction of $\operatorname{Aut}(A)$.

When the group $A$ is finite, the parameter set $\Gamma(G, A)$ is of course also finite. Efficient counting methods for determining the size of this set were pioneered by P. Hall in the 1930s. New techniques (involving, among other things, characteristic varieties over finite fields) were introduced in [10]. In the particular case when $A$ is a finite Abelian group, a closed formula for the cardinality of $\Gamma(G, A)$ was given in [10], see Theorem 2.3 below.

\subsection{Functoriality properties}

The above construction enjoys some (partial) functoriality properties in both arguments. First, suppose that $\varphi: G_{1} \rightarrow G_{2}$ is an epimorphism between two groups. Composition with $\varphi$ gives a map $\operatorname{Epi}\left(G_{2}, A\right) \rightarrow \operatorname{Epi}\left(G_{1}, A\right)$, which in turn induces a well-defined map,

$$
\Gamma\left(G_{2}, A\right) \stackrel{\varphi^{*}}{\longrightarrow} \Gamma\left(G_{1}, A\right), \quad[\nu] \mapsto[\nu \circ \varphi]
$$

Under the correspondence from Lemma 2.1, this map can be interpreted as follows. Let $f:\left(X_{1}, x_{1}\right) \rightarrow\left(X_{2}, x_{2}\right)$ be a basepoint-preserving map between connected $\mathrm{CW}$-complexes, and suppose $f$ induces an epimorphism $\varphi=f_{\sharp}: G_{1} \rightarrow G_{2}$ on fundamental groups. Then the map $\varphi^{*}$ sends the equivalence class of the cover $p_{\nu}: X_{2}^{v} \rightarrow X_{2}$ to that of the pull-back cover, $p_{\nu \circ \varphi}=f^{*}\left(p_{\nu}\right): X_{1}^{\nu \circ \varphi} \rightarrow X_{1}$.

Next, recall that a subgroup $K<A$ is characteristic if $\alpha(K)=K$, for all $\alpha \in \operatorname{Aut}(A)$.

Lemma 2.2. Suppose we have an exact sequence of groups, $1 \rightarrow K \rightarrow A \stackrel{\pi}{\rightarrow}$ $B \rightarrow 1$, with $K$ a characteristic subgroup of $A$. There is then a well-defined map between the parameter sets for regular A-covers and B-covers of $X$,

$$
\Gamma(G, A) \stackrel{\tilde{\pi}}{\longrightarrow} \Gamma(G, B)
$$

which sends $[v]$ to $[\pi \circ v]$. 
Proof. Suppose $v_{1}, v_{2}: G \rightarrow A$ are two epimorphisms so that $\alpha \circ v_{1}=v_{2}$, for some $\alpha \in \operatorname{Aut}(A)$. Since the subgroup $K=\operatorname{ker}(\pi)$ is characteristic, the automorphism $\alpha$ induces and automorphism $\bar{\alpha} \in \operatorname{Aut}(B)$ such that $\bar{\alpha} \circ \pi=\pi \circ \alpha$. Hence, $\bar{\alpha} \circ\left(\pi \circ \nu_{1}\right)=$ $\pi \circ v_{2}$, showing that $q$ is well-defined.

The correspondence of Lemmas 2.1 and 2.2 is summarized in the following diagram:
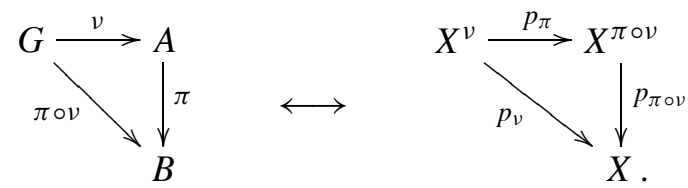

Under this correspondence, the map $\tilde{\pi}$ sends the equivalence class of the cover $p_{v}$ to that of the cover $p_{\pi \circ \mathrm{v}}$.

\subsection{Regular Abelian covers}

Let $H=G^{\text {ab }}$ be the Abelianization of our group $G$. Recall we are assuming $G$ is finitely generated; thus, $H$ is a finitely generated Abelian group.

Now suppose $A$ is any other (finitely generated) Abelian group. In this case, every homomorphism $G \rightarrow A$ factors through the Abelianization map, ab: $G \rightarrow$ $H$. Composition with this map gives a bijection between $\operatorname{Epi}(H, A)$ and $\operatorname{Epi}(G, A)$, which induces a bijection

$$
\Gamma(H, A) \stackrel{\cong}{\longrightarrow} \Gamma(G, A), \quad[v] \mapsto[v \circ \mathrm{ab}] .
$$

In view of Lemma 2.1, we obtain a bijection between the set of equivalence classes of connected, regular $A$-covers of a CW-complex $X$ and the set $\Gamma(H, A)$, where $H=H_{1}(X, \mathbb{Z})$ is the Abelianization of $G=\pi_{1}\left(X, x_{0}\right)$. Note that this parameter set is empty, unless $A$ is a quotient of $H$.

Let Tors $(A)$ be the torsion subgroup, consisting of finite-order elements in $A$; clearly, this is a characteristic subgroup of $A$. Let $\bar{A}=A$ / Tors $(A)$ be the quotient group, and let $\pi: A \rightarrow \bar{A}$ be the canonical projection.

Under the correspondence from (2.4), an epimorphism $v: H \rightarrow A$ determines a regular cover, $p_{\nu \circ a b}: X^{\nu \circ a b} \rightarrow X$, which, for economy of notation, we will write as $p_{\nu}: X^{v} \rightarrow X$. There is also a free Abelian cover, $p_{\bar{v}}: X^{\bar{v}} \rightarrow X$, corresponding to the epimorphism $\bar{v}=\pi \circ v: H \rightarrow \bar{A}$.

The projection $\pi: A \rightarrow \bar{A}$ defines a map $\operatorname{Epi}(H, A) \rightarrow \operatorname{Epi}(H, \bar{A}), v \mapsto \bar{v}$, which in turn induces maps between the parameter spaces for $A$ and $\bar{A}$ covers,

$$
\Gamma(H, A) \stackrel{q_{H}}{\longrightarrow} \Gamma(H, \bar{A}),
$$

sending the cover $p_{v}$ to the cover $p_{\bar{v}}$. Notice that this map is compatible with the morphism $\tilde{\pi}$ from (2.2), induced by an epimorphism $\pi: A \rightarrow B$ with characteristic kernel. 


\subsection{Splitting the torsion-free part}

For a topological group $G$, let $G \rightarrow E G \rightarrow B G$ be the universal principal $G$ bundle; the total space $E G$ a contractible $\mathrm{CW}$-complex endowed with a free $G$ action, while the base space $B G$ the quotient space under this action. We will only consider here the situation when $G$ is discrete, in which case $B G=K(G, 1)$ and $E G=\widehat{K(G, 1)}$.

As before, let $H$ be a finitely generated Abelian group. Let Tors $(H)$ be its torsion subgroup, and let $\bar{H}=H / \operatorname{Tors}(H)$. Fix a splitting $\bar{H} \rightarrow H$; then $H \cong$ $\bar{H} \oplus \operatorname{Tors}(H)$.

Now consider an epimorphism $v: H \rightarrow A$. After fixing a splitting $\bar{A} \hookrightarrow A$, we obtain a decomposition $A \cong \bar{A} \oplus \operatorname{Tors}(A)$. We may view $\bar{A}$ as a subgroup of $H$ by choosing a splitting $\bar{A} \hookrightarrow H$ of the projection $H \rightarrow \bar{A}$. With these identifications, $v$ induces an epimorphism $\tilde{v}: H / \bar{A} \rightarrow A / \bar{A}$. This observation leads us to consider the set

$$
\Gamma=\Gamma(H / \bar{A}, A / \bar{A}) .
$$

Clearly, the set $\Gamma$ is finite. Theorem 3.1 from [10] yields an explicit formula for the size of this set. Given a finite Abelian group $K$, and a prime $p$, write the $p$-torsion part of $K$ as $K_{p}=\mathbb{Z}_{p^{\lambda_{1}}} \oplus \cdots \oplus \mathbb{Z}_{p^{\lambda_{s}}}$, for some positive integers $\lambda_{1} \geq \cdots \geq \lambda_{s}$, where $s=0$ if $p \nmid|K|$. Thus, $K_{p}$ determines a partition $\pi\left(K_{p}\right)=\left(\lambda_{1}, \ldots, \lambda_{s}\right)$. For each such partition $\lambda$, write $l(\lambda)=s,|\lambda|=\sum_{i=1}^{s} \lambda_{i}$, and $\langle\lambda\rangle=\sum_{i=1}^{s}(i-1) \lambda_{i}$.

Theorem 2.3 ([10]). Set $n=\operatorname{rank} H$ and $r=\operatorname{rank} A$. For each prime $p$ dividing the order of $A / \bar{A}$, let $\lambda=\pi\left((A / \bar{A})_{p}\right)$ and $\tau=\pi\left((\operatorname{Tors}(H / \bar{A}))_{p}\right)$ be the corresponding partitions. Then,

$$
|\Gamma(H / \bar{A}, A / \bar{A})|=\prod_{p|| A / \bar{A} \mid} \frac{p^{(|\lambda|-l(\lambda))(n-r)+\theta\left(\lambda^{-}, \tau\right)} \prod_{i=1}^{l(\lambda)}\left(p^{n-r+\theta_{i}(\lambda, \tau)-\theta_{i}\left(\lambda^{-}, \tau\right)}-p^{i-1}\right)}{p^{|\lambda|+2\langle\lambda\rangle} \prod_{k \geq 1} \varphi_{m_{k}(\lambda)}\left(p^{-1}\right)},
$$

where $m_{k}(\lambda)=\#\left\{i \mid \lambda_{i}=k\right\}, \varphi_{m}(t)=\prod_{i=1}^{m}\left(1-t^{i}\right), \lambda^{-}$is the partition with $\lambda_{i}^{-}=\lambda_{i}-1, \theta_{i}(\lambda, \tau)=\sum_{j=1}^{l(\tau)} \min \left(\lambda_{i}, \tau_{j}\right)$, and $\theta(\lambda, \tau)=\sum_{i=1}^{l(\lambda)} \theta_{i}(\lambda, \tau)$.

For instance, $\left|\Gamma\left(\mathbb{Z}^{n}, \mathbb{Z}_{p^{s}}\right)\right|=\frac{p^{s n}-p^{(s-1) n}}{p^{s}-p^{s-1}}$, whereas $\left|\Gamma\left(\mathbb{Z}^{n}, \mathbb{Z}_{p}^{s}\right)\right|=\prod_{i=0}^{s-1} \frac{p^{n}-p^{i}}{p^{s}-p^{i}}$.

Each homomorphism $H / \bar{A} \rightarrow A / \bar{A}$ defines an action of $H / \bar{A}$ on $A / \bar{A}$. This action yields a fiber bundle,

$$
A / \bar{A} \rightarrow E(H / A) \times_{H / \bar{A}} A / \bar{A} \rightarrow B(H / \bar{A})
$$

associated to the principal bundle $H / \bar{A} \rightarrow E(H / \bar{A}) \rightarrow B(H / \bar{A})$. The set $\Gamma=$ $\Gamma(H / \bar{A}, A / \bar{A})$ parameterizes all such associated bundles. 


\subsection{A pullback diagram}

We return now to diagram (2.5), which relates the parameter sets for regular $A$ and $\bar{A}$ covers of a connected CW-complex $X$. This diagram can be further analyzed by using pullbacks from the universal principal bundles over the classifying spaces for the discrete groups $A$ and $\bar{A}$.

Let $A \rightarrow E A \rightarrow B A$ and $\bar{A} \rightarrow E \bar{A} \rightarrow B \bar{A}$ be the respective classifying bundles, and let $X \rightarrow B A$ and $X \rightarrow B \bar{A}$ be classifying maps for the covers $X^{v} \rightarrow$ $X$ and $X^{\bar{v}} \rightarrow X$, respectively. Upon identifying Tors $(A)$ with $A / \bar{A}$, we obtain the following diagram:

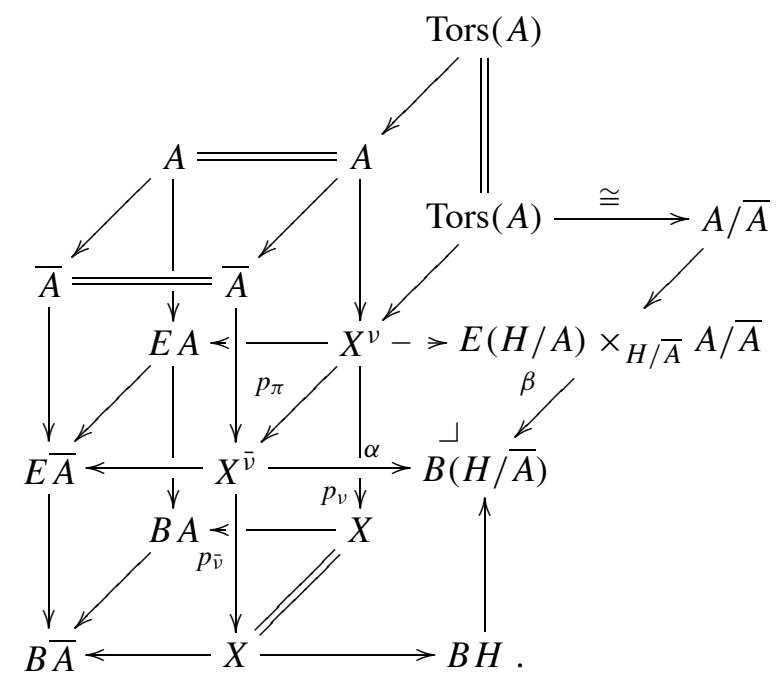

Here, the map $X \rightarrow B H$ realizes the Abelianization morphism, ab: $\pi_{1}\left(X, x_{0}\right) \rightarrow$ $H$, while $\alpha$ denotes the composite $X^{\bar{v}} \rightarrow X \rightarrow B H \rightarrow B(H / \bar{A})$.

Proposition 2.4. The marked square in diagram (2.8) is a pullback square. That is, the cover $p_{\pi}: X^{\nu} \rightarrow X^{\bar{v}}$ is the pullback along the map $\alpha: X^{\bar{v}} \rightarrow B(H / \bar{A})$ of the cover $\beta: E(H / A) \times_{H / \bar{A}} A / \bar{A} \rightarrow B(H / \bar{A})$ corresponding to the epimorphism $\tilde{v}: H / \bar{A} \rightarrow A / \bar{A}$.

Proof. Clearly, $\left(\alpha \circ p_{\pi}\right)_{\sharp}\left(\pi_{1}\left(X^{v}\right)\right)=\operatorname{im}(\operatorname{ker} v \hookrightarrow \operatorname{ker} \bar{v})$, while $\beta_{\sharp}\left(\pi_{1}\left(E(H / A) \times_{H / A}\right.\right.$ $A / \bar{A}))=\operatorname{ker}(\tilde{v}: H / \bar{A} \rightarrow A / \bar{A})$. After picking a splitting $\bar{A} \hookrightarrow H$, and identifying the group $H$ with $\operatorname{ker} \bar{v} \oplus \bar{A}$, we see that

$$
\left(\alpha \circ p_{\pi}\right)_{\sharp}\left(\pi_{1}\left(X^{v}\right)\right) \subseteq \beta_{\sharp}\left(\pi_{1}\left(E(H / A) \times_{H / \bar{A}} A / \bar{A}\right)\right) .
$$

The existence of the dashed arrow in the diagram follows then from the lifting criterion for covers. It is readily seen that this arrow is equivariant with respect to the actions on source and target by $\operatorname{Tors}(A)$ and $A / \bar{A}$; thus, a morphism of covers. This completes the proof. 
Using this proposition, and the discussion from Section 2.4, we obtain the following corollary.

Corollary 2.5. With notation as above,

$$
\Gamma(H / \bar{A}, A / \bar{A}) \longrightarrow \Gamma(H, A) \stackrel{q_{H}}{\longrightarrow} \Gamma(H, \bar{A})
$$

is a set fibration; that is, all fibers of $q_{H}$ are in bijection with the set $\Gamma(H / \bar{A}, A / \bar{A})$.

Let us identify topologically the fiber of $q_{H}$. A regular, $\bar{A}$-cover of our space $X$ corresponds to an epimorphism $\bar{v}: H \rightarrow \bar{A}$. A regular, Tors $(A)$-cover of $X^{\bar{v}}$ corresponds to an epimorphism $\operatorname{ker} \bar{v} \rightarrow \operatorname{Tors}(A)$. Given these data, and the chosen splitting $\bar{A} \hookrightarrow A$, we can find an epimorphism $v: H \rightarrow A$, such that the following diagram commutes:

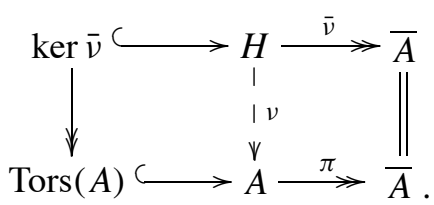

Thus, any regular, Tors $(A)$-cover $X^{v} \rightarrow X^{\bar{v}}$ defines a regular $A$-cover $X^{v} \rightarrow X$, whose corresponding free Abelian cover is $X^{\bar{\nu}}$. Consequently, the fiber of $[\bar{v}]$ under the map $q_{H}: \Gamma(H, A) \rightarrow \Gamma(H, \bar{A})$ coincides with the set

$$
\left\{[v] \in \Gamma(H, A) \mid X^{v} \text { is a regular Tors }(A) \text {-cover of } X^{\bar{v}}\right\} .
$$

\section{Reinterpreting the parameter set for $A$-covers}

In this section, we give a geometric description of the parameter set for regular Abelian covers of a space.

\subsection{Splittings}

As before, let $H$ and $A$ be finitely generated Abelian groups, and assume there is an epimorphism $H \rightarrow A$.

Lemma 3.1. The action $\operatorname{Aut}(A)$ on the set of all splittings $A / \operatorname{Tors}(A) \hookrightarrow A$ induced by the natural action of $\operatorname{Aut}(A)$ on $A$ is transitive.

Proof. Set $\bar{A}=A$ / Tors $(A)$, and fix a splitting $s: \bar{A} \hookrightarrow A$. Using this splitting, we may decompose the group $A$ as $\bar{A} \oplus \operatorname{Tors}(A)$, and view $s: \bar{A} \hookrightarrow \bar{A} \oplus \operatorname{Tors}(A)$ as the map $a \mapsto(a, 0)$.

An arbitrary splitting $\sigma: \bar{A} \hookrightarrow \bar{A} \oplus$ Tors $(A)$ is given by $a \mapsto\left(a, \sigma_{2}(a)\right)$, for some homomorphism $\sigma_{2}: \bar{A} \rightarrow \operatorname{Tors}(A)$. Consider the automorphism of $\alpha \in$ $\operatorname{Aut}(\bar{A} \oplus \operatorname{Tors}(A))$ given by the matrix $\left(\begin{array}{ll}\text { id } & 0 \\ \sigma_{2} & \text { id }\end{array}\right)$. Clearly, $\alpha \circ s=\sigma$, and we are done. 
Denote by $n$ the rank of $H$, and by $r$ the rank of $A$. Fixing splittings $\bar{H} \hookrightarrow H$ and $\bar{A} \hookrightarrow A$, we have $H=\bar{H} \oplus \operatorname{Tors}(H)$, with $\bar{H}=\mathbb{Z}^{n}$, and $A=\bar{A} \oplus \operatorname{Tors}(A)$, with $\bar{A}=\mathbb{Z}^{r}$.

Now identify the automorphism group $\operatorname{Aut}(\bar{H})$ with the general linear group $\mathrm{GL}_{n}(\mathbb{Z})$. Let $\mathrm{P}$ be the parabolic subgroup of $\mathrm{GL}_{n}(\mathbb{Z})$, consisting all matrices of the form $\left(\begin{array}{cc}*_{1} & *_{2} \\ 0 & *_{3}\end{array}\right)$, where $*_{1}$ is of size $(n-r) \times(n-r)$. Then $\mathrm{GL}_{n}(\mathbb{Z}) / \mathrm{P}$ is isomorphic to the Grassmannian $\mathrm{Gr}_{n-r}\left(\mathbb{Z}^{n}\right)$. It is readily checked that the left action of $\mathrm{P}$ on $\mathbb{Z}^{n-r}$, given by multiplication of $\left\{*_{1}\right\} \cong \mathrm{GL}_{n-r}(\mathbb{Z})$ on $\mathbb{Z}^{n-r}$, induces an action of $\mathrm{P}$ on the set $\Gamma=\Gamma(H / \bar{A}, A / \bar{A})$. Also note that, if $A$ is torsion-free, then the set $\Gamma$ is a singleton.

\subsection{A fibered product}

We are now ready to state and prove the main result of this section.

Theorem 3.2. There is a bijection

$$
\Gamma(H, A) \longleftrightarrow \mathrm{GL}_{n}(\mathbb{Z}) \times{ }_{\mathrm{P}} \Gamma
$$

between the parameter set $\Gamma(H, A)=\operatorname{Epi}(H, A) / \operatorname{Aut}(A)$ and the twisted product of $\mathrm{GL}_{n}(\mathbb{Z})$ with the set $\Gamma=\Gamma(H / \bar{A}, A / \bar{A})$ over the parabolic subgroup $\mathrm{P}$. Under this bijection, the map $q: \Gamma(H, A) \rightarrow \Gamma(H, \bar{A})$ induced by the projection $\pi: A \rightarrow$ $\bar{A}$ corresponds to the canonical projection

$$
\mathrm{GL}_{n}(\mathbb{Z}) \times_{\mathrm{P}} \Gamma \rightarrow \mathrm{GL}_{n}(\mathbb{Z}) / \mathrm{P}=\mathrm{Gr}_{n-r}\left(\mathbb{Z}^{n}\right) .
$$

Proof. Define a map $\theta: \mathrm{GL}_{n}(\mathbb{Z}) \times \Gamma \rightarrow \Gamma(H, A)$ as follows. Given an element $(M,[\gamma])$ of $\mathrm{GL}_{n}(\mathbb{Z}) \times \Gamma$, let $\left(\gamma_{1}, \gamma_{2}\right)$ be a representative of $[\gamma]$, with $\gamma_{1}: \mathbb{Z}^{n-r} \rightarrow$ $\operatorname{Tors}(A)$ and $\gamma_{2}$ : Tors $(H) \rightarrow \operatorname{Tors}(A)$. Let $\alpha_{1}, \ldots, \alpha_{n}$ be the column vectors of the matrix $M$, which forms a basis of $\bar{H} \cong \mathbb{Z}^{n}$, we can write $H=\mathbb{Z}^{n-r} \oplus \mathbb{Z}^{r} \oplus \operatorname{Tors}(H)$, where $\mathbb{Z}^{n-r}$ is the subspace of $\bar{H}$ generated by the first $n-r$ column vectors of $M$. Now define $\theta(M,[\gamma])=[v]$, where $v: \mathbb{Z}^{n-r} \oplus \mathbb{Z}^{r} \oplus \operatorname{Tors}(H) \rightarrow \mathbb{Z}^{r} \oplus \operatorname{Tors}(A)$ is the homomorphism given by the matrix $N=\left(\begin{array}{ccc}0 & \text { id } & 0 \\ \gamma_{1} & 0 & \gamma_{2}\end{array}\right)$. It is straightforward to check that the map $\theta$ is well-defined, i.e., $\theta$ is independent of the splitting and representative we chose.

Now let's check that the map $\theta$ factors through $\mathrm{GL}_{n}(\mathbb{Z}) \times{ }_{\mathrm{P}} \Gamma$. Suppose we have two elements $\left(M,\left[\gamma_{1}, \gamma_{2}\right]\right)$ and $\left(M^{\prime},\left[\gamma_{1}^{\prime}, \gamma_{2}^{\prime}\right]\right)$ of $\mathrm{GL}_{n}(\mathbb{Z}) \times \Gamma$ which are equivalent, that is, there is a matrix $Q=\left(\begin{array}{cc}Q_{1} & Q_{2} \\ 0 & Q_{3}\end{array}\right) \in \mathrm{P}$ such that $M=M^{\prime} Q$ and $\left(\gamma_{1} Q_{1}^{-1}, \gamma_{2}\right)=$ $\left(\gamma_{1}^{\prime}, \gamma_{2}^{\prime}\right)$. By definition, the map $\theta$ takes the pair $\left(M,\left[\gamma_{1}, \gamma_{2}\right]\right)$ to the homomorphism $v$ given by the matrix $N$ above. Changing the basis of $\bar{H}$ to the basis given by the column vectors of $M^{\prime}$, the map $v$ is given by the matrix

$$
N Q^{-1}=\left(\begin{array}{ccc}
0 & Q_{3}^{-1} & 0 \\
\gamma_{1} Q_{1}^{-1} & \gamma_{1} Q_{4} & \gamma_{2}
\end{array}\right)=\left(\begin{array}{cc}
Q_{3}^{-1} & 0 \\
\gamma_{1} Q_{4} & \text { id }
\end{array}\right)\left(\begin{array}{ccc}
0 & \text { id } & 0 \\
\gamma_{1}^{\prime} & 0 & \gamma_{2}^{\prime}
\end{array}\right) .
$$


Clearly, the matrix $\left(\begin{array}{cc}Q_{3}^{-1} & 0 \\ \gamma_{1} Q_{4} & \text { id }\end{array}\right)$ defines an automorphism of $A$. Thus, the map $\theta$ factors through a well-defined map, $\theta: \mathrm{GL}_{n}(\mathbb{Z}) \times_{\mathrm{P}} \Gamma \rightarrow \Gamma(H, A)$, which is readily seen to be a bijection. It is now a straightforward matter to verify the last assertion.

\subsection{Further discussion}

A particular case of the above theorem is worth singling out. Recall $\bar{H}=\mathbb{Z}^{n}$ and $\bar{A}=\mathbb{Z}^{r}$.

Corollary 3.3. Suppose Tors $(H)=\mathbb{Z}_{p}^{s}$ and Tors $(A)=\mathbb{Z}_{p}^{t}$, for some prime $p$. Then, the parameter set $\Gamma(H, A)$ is in bijective correspondence with the set $\mathrm{GL}_{n}(\mathbb{Z}) \times \mathrm{P}_{\mathrm{P}} \mathrm{Gr}_{t}\left(\mathbb{Z}_{p}^{n-r+s}\right)$.

Proof. In this case, the set $\Gamma=\Gamma(H / \bar{A}, A / \bar{A})$ is in bijection with the set $\operatorname{Epi}\left(\mathbb{Z}_{p}^{n-r} \oplus \mathbb{Z}_{p}^{s}, \mathbb{Z}_{p}^{t}\right) / \operatorname{Aut}\left(\mathbb{Z}_{p}^{t}\right)$. This bijection is established using the diagram

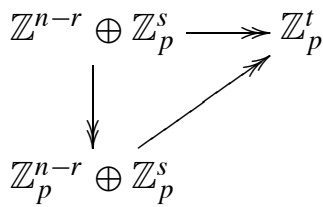

Therefore, $\Gamma=\mathrm{Gr}_{t}\left(\mathbb{Z}_{p}^{n-r+s}\right)$, and we are done.

Remark 3.4. Consider the projection map $q: \mathrm{GL}_{n}(\mathbb{Z}) \times_{\mathrm{P}} \Gamma \rightarrow \mathrm{GL}_{n}(\mathbb{Z}) / \mathrm{P}=$ $\operatorname{Gr}_{n-r}\left(\mathbb{Z}^{n}\right)$ from Theorem 3.2. It is readily seen that, for each subspace $Q \in$ $\operatorname{Gr}_{n-r}\left(\mathbb{Z}^{n}\right)$, the cardinality of the fiber $q^{-1}(Q)$ is the same as the the cardinality of the set $\Gamma$. In particular, $q^{-1}(Q)$ is finite, for all $Q \in \mathrm{Gr}_{n-r}\left(\mathbb{Z}^{n}\right)$.

\section{Dwyer-Fried sets and their generalizations}

In this section, we define a sequence of subsets $\Omega_{A}^{i}(X)$ of the parameter set for regular $A$-covers of $X$. These sets, which generalize the Dwyer-Fried sets $\Omega_{r}^{i}(X)$, keep track of the homological finiteness properties of those covers.

\subsection{Generalized Dwyer-Fried sets}

Throughout this section, $X$ will be a connected CW-complex with finite 1-skeleton, and $G=\pi_{1}\left(X, x_{0}\right)$ will denote its fundamental group.

Definition 4.1. For each group $A$ and integer $i \geq 0$, the corresponding DwyerFried set of $X$ is defined as

$$
\Omega_{A}^{i}(X)=\left\{[v] \in \Gamma(G, A) \mid b_{j}\left(X^{v}\right)<\infty, \text { for all } 0 \leq j \leq i\right\} .
$$


In other words, the sets $\Omega_{A}^{i}(X)$ parameterize those regular $A$-covers of $X$ having finite Betti numbers up to degree $i$. In the particular case when $A$ is a free Abelian group of rank $r$, we recover the standard Dwyer-Fried sets, $\Omega_{r}^{i}(X)=\Omega_{\mathbb{Z}^{r}}^{i}(X)$, viewed as subsets of the Grassmannian $\operatorname{Gr}_{r}\left(\mathbb{Q}^{n}\right)$, where $n=b_{1}(X)$. By our assumption on the 1-skeleton on $X$, the group $G=\pi_{1}\left(X, x_{0}\right)$ is finitely generated. Thus, we may assume $A$ is also finitely generated, for otherwise $\operatorname{Epi}(G, A)=\varnothing$, and so $\Omega_{A}^{i}(X)=\emptyset$, too. The $\Omega$-sets are invariant under homotopy equivalence. More precisely, we have the following lemma, which generalizes the analogous lemma for free Abelian covers, proved in [14].

Lemma 4.2. Let $f: X \rightarrow Y$ be a (cellular) homotopy equivalence. For any group $A$, the homomorphism $f_{\sharp}: \pi_{1}\left(X, x_{0}\right) \rightarrow \pi_{1}\left(Y, y_{0}\right)$ induces a bijection $f_{\sharp}^{*}: \Gamma\left(\pi_{1}\left(Y, y_{0}\right), A\right) \rightarrow \Gamma\left(\pi_{1}\left(X, x_{0}\right), A\right)$, sending each subset $\Omega_{A}^{i}(Y)$ bijectively onto $\Omega_{A}^{i}(X)$.

Proof. Since $f$ is a homotopy equivalence, the induced homomorphism on fundamental groups, $f_{\sharp}$, is a bijection. Thus, the corresponding map between parameter sets, $f_{\sharp}^{*}$, is a bijection. To finish the proof, it remains to verify that $f_{\sharp}^{*}\left(\Omega_{A}^{i}(Y)\right)=$ $\Omega_{A}^{i}(X)$

Let $v: \pi_{1}\left(Y, y_{0}\right) \rightarrow A$ be an epimorphism. Composing with $f_{\sharp}$, we get an epimorphism $\nu \circ f_{\sharp}: \pi_{1}\left(X, x_{0}\right) \rightarrow A$. By the lifting criterion, $f$ lifts to a map $\bar{f}$ between the respective $A$-covers. This map fits into the following pullback diagram:

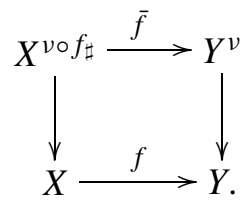

Clearly, $\bar{f}: X^{\nu \circ f_{\sharp}} \rightarrow Y^{\nu}$ is also a homotopy equivalence. Thus, $b_{j}\left(Y^{v}\right)<\infty$ if and only if $b_{j}\left(X^{\nu \circ f_{\sharp}}\right)<\infty$, which means that $f_{\sharp}^{*}\left(\Omega_{A}^{i}(Y)\right)=\Omega_{A}^{i}(X)$.

Based on this lemma, we may define the $\Omega$-sets of a (discrete, finitely generated) group $G$ as $\Omega_{A}^{i}(G):=\Omega_{A}^{i}(B G)$, where $B G=K(G, 1)$ is a classifying space for $G$.

\subsection{Naturality properties}

The Dwyer-Fried sets (or their complements) enjoy certain naturality properties in both variables, which we now describe.

Proposition 4.3. Let $\varphi: G \rightarrow Q$ be an epimorphism of groups. Then, for each group $A$, there is an inclusion $\Omega_{A}^{1}(Q)^{c} \hookrightarrow \Omega_{A}^{1}(G)^{c}$.

Proof. Let $v: Q \rightarrow A$ be an epimorphism. Composing with $\varphi$, we get an epimorphism $\nu \circ \varphi: G \rightarrow A$. So there is an epimorphism $\varphi: \operatorname{ker}(\nu \circ \varphi) \rightarrow \operatorname{ker}(\nu)$. Taking 
Abelianizations, we get an epimorphism $\varphi_{\mathrm{ab}}: \operatorname{ker}(\nu \circ \varphi)_{\mathrm{ab}} \rightarrow \operatorname{ker}(\nu)_{\mathrm{ab}}$. Thus, if $\operatorname{ker}(v)_{\mathrm{ab}}$ has infinite rank, then $\operatorname{ker}(\nu \circ \varphi)_{\mathrm{ab}}$ also has infinite rank. The desired conclusion follows.

Before proceeding, let us recall a well-known result regarding the homology of finite covers, which can be proved via a standard transfer argument (see, for instance, [8]).

Lemma 4.4. Let $p: Y \rightarrow X$ be a regular cover defined by a properly discontinuous action of a finite group $A$ on $Y$, and let $\mathbb{k}$ be a coefficient field of characteristic 0 , or a prime not dividing the order of $A$. Then, the induced homomorphism in cohomology, $p^{*}: H^{*}(X ; \mathbb{k}) \rightarrow H^{*}(Y ; \mathbb{k})$, is injective, with image the subgroup $H^{*}(Y ; \mathbb{k})^{A}$ consisting of those classes $\alpha$ for which $\gamma^{*}(\alpha)=\alpha$, for all $\gamma \in A$.

Corollary 4.5. Let $p: Y \rightarrow X$ be a finite, regular cover. Then $b_{i}(X) \leq b_{i}(Y)$, for all $i \geq 0$.

Now fix a CW-complex $X$ as above, with fundamental group $G=\pi_{1}\left(X, x_{0}\right)$. Suppose $1 \rightarrow K \rightarrow A \stackrel{\pi}{\rightarrow} B \rightarrow 1$ is a short exact sequence of groups, with $K$ a characteristic subgroup of $A$. As noted in Lemma 2.2, the homomorphism $\pi$ induces a map $\tilde{\pi}: \Gamma(G, A) \rightarrow \Gamma(G, B),[\nu] \mapsto[\pi \circ \nu]$, between the parameter sets for regular $A$-covers and $B$-covers of $X$.

Proposition 4.6. Suppose $K=\operatorname{ker}(\pi: A \rightarrow B)$ is a finite, characteristic subgroup of $A$. Then the map $\tilde{\pi}: \Gamma(G, A) \rightarrow \Gamma(G, B)$ restricts to a map $\tilde{\pi}: \Omega_{A}^{i}(X) \rightarrow$ $\Omega_{B}^{i}(X)$ between the respective Dwyer-Fried sets.

Proof. Let $v: G \rightarrow A$ be an epimorphism, and suppose $[v] \in \Omega_{A}^{i}(X)$, that is, $b_{j}\left(X^{v}\right)<\infty$, for all $j \leq i$. Then $X^{v} \rightarrow X^{\pi \circ v}$ is a regular $K$-cover. By Corollary 4.5, we have that $b_{j}\left(X^{\pi \circ v}\right)<\infty$, for all $j \leq i$; in other words, $[\pi \circ \nu] \in \Omega_{B}^{i}(X)$.

Proposition 4.6 may be summarized in the following commuting diagram:

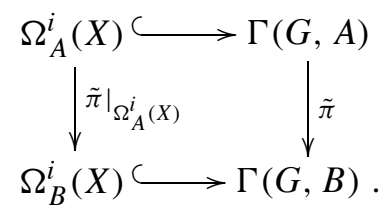

This diagram is a pullback diagram precisely when

$$
\tilde{\pi}^{-1}\left(\Omega_{B}^{i}(X)\right)=\Omega_{A}^{i}(X) .
$$

As we shall see later on, this condition is not always satisfied. For now, let us just single out a simple situation when (4.2) is tautologically a pullback diagram.

Corollary 4.7. With notation as above, if $\Omega_{B}^{i}(X)=\emptyset$, then $\Omega_{A}^{i}(X)=\emptyset$. 


\subsection{Abelian versus free Abelian covers}

Let us now consider in more detail the case when $A$ is an Abelian group. As usual, we are only interested in the case when $A$ is a quotient of the (finitely generated) group $H=G_{\mathrm{ab}}$, and thus we may assume $A$ is also finitely generated.

Consider the exact sequence $0 \rightarrow \operatorname{Tors}(A) \rightarrow A \rightarrow \bar{A} \rightarrow 0$. Clearly, Tors $(A)$ is a finite, characteristic subgroup of $A$. Thus, Proposition 4.6 applies, giving a map

$$
q: \Omega_{A}^{i}(X) \rightarrow \Omega_{\bar{A}}^{i}(X) .
$$

In particular, if $\Omega_{\bar{A}}^{i}(X)=\emptyset$, then $\Omega_{A}^{i}(X)=\emptyset$.

Example 4.8. Let $\Sigma_{g}$ be a Riemann surface of genus $g \geq 2$. It is readily seen that $\Omega_{r}^{i}\left(\Sigma_{g}\right)=\emptyset$, for all $r \geq 1$ and $i \geq 1, c f$. [14]. Thus, if $A$ is any finitely generated Abelian group with rank $A \geq 1$, then $\Omega_{A}^{i}\left(\Sigma_{g}\right)=\emptyset$, for all $i \geq 1$.

Suppose now we have a short exact sequence $1 \rightarrow K \rightarrow A \stackrel{\pi}{\rightarrow} B \rightarrow 1$, with $K$ characteristic. Let $\bar{\pi}: \bar{A} \rightarrow \bar{B}$ be the induced epimorphism between maximal torsion-free quotients. Since $K=\operatorname{ker}(\pi)$ is finite, $\bar{\pi}$ is an isomorphism. Using Proposition 4.6 again, and the identification from (2.4), we obtain the following commutative diagram:

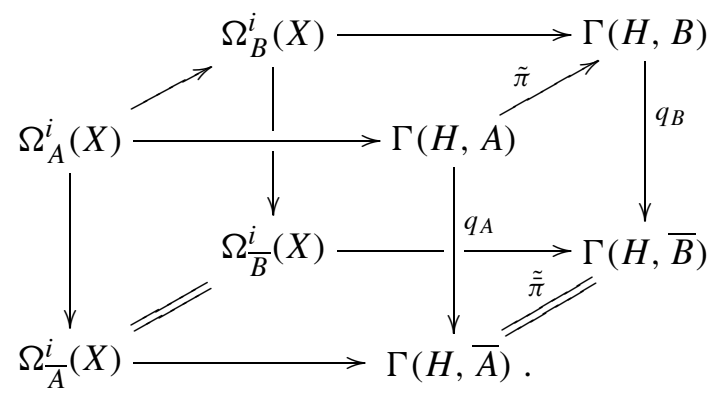

Proposition 4.9. Assume the function $\tilde{\pi}: \Gamma(H, A) \rightarrow \Gamma(H, B)$ is surjective. Then, if the front square in diagram (4.5) is a pullback square, so is the back square; that is,

$$
q_{A}^{-1}\left(\Omega_{\bar{A}}^{i}\right)=\Omega_{A}^{i} \Longrightarrow q_{B}^{-1}\left(\Omega_{\bar{B}}^{i}\right)=\Omega_{B}^{i} .
$$

Proof. Suppose the back square is not a pullback square. Then there exist elements $[\bar{v}] \in \Omega_{\bar{B}}^{i}$ and $[\nu] \in \Gamma(H, B) \backslash \Omega_{B}^{i}$ such that $q_{B}([v])=\bar{v}$. By assumption, the map $\tilde{\pi}$ is surjective; thus, $\tilde{\pi}^{-1}([v])$ is nonempty. Pick an element $[\sigma] \in \tilde{\pi}^{-1}([v])$. Then $[\sigma] \in \Gamma(H, A) \backslash \Omega_{A}^{i}$, for otherwise $[v]=\tilde{\pi}([\sigma]) \in \Omega_{B}^{i}$. On the other hand,

$$
q_{A}([\sigma])=q_{B}(\tilde{\pi}([\sigma]))=q_{B}([\nu])=[\bar{v}] \in \Omega_{\bar{B}}^{i}=\Omega_{\frac{i}{A}} .
$$

Thus, the front square is not a pullback diagram, either. 


\subsection{The comparison diagram}

Now fix a splitting $\bar{A} \hookrightarrow A$, which gives rise to an isomorphism $A \cong \bar{A} \oplus \operatorname{Tors}(A)$. Similarly, after fixing a splitting $\bar{H} \hookrightarrow H$, the Abelianization $H=G_{\mathrm{ab}}$ also decomposes as $H \cong \bar{H} \oplus \operatorname{Tors}(H)$. Theorem 3.2 yields an identification

$$
\Gamma(H, A) \cong \mathrm{GL}_{n}(\mathbb{Z}) \times_{\mathrm{P}} \Gamma
$$

where $n=\operatorname{rank} H$, the group $\mathrm{P}$ is a parabolic subgroup of $\mathrm{GL}_{n}(\mathbb{Z})$ so that $\mathrm{GL}_{n}(\mathbb{Z}) / \mathrm{P}=\mathrm{Gr}_{n-r}\left(\mathbb{Z}^{n}\right)$, and $\Gamma=\Gamma(H / \bar{A}, A / \bar{A})$.

Putting things together, we obtain a commutative diagram, which we shall refer to as the comparison diagram,

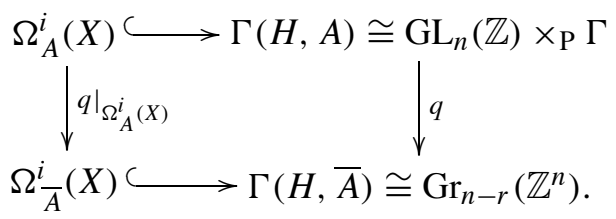

The next result reinterprets the condition that this diagram is a pull-back in terms of Betti numbers of Abelian covers.

Proposition 4.10. The following conditions are equivalent:

(i) Diagram (4.7) is a pull-back diagram.

(ii) $q^{-1}\left(\Omega_{\bar{A}}^{i}(X)\right)=\Omega_{A}^{i}(X)$.

(iii) If $X^{\bar{v}}$ is a regular $\bar{A}$-cover with finite Betti numbers up to degree $i$, then any regular Tors $(A)$-cover of $X^{\bar{v}}$ has the same finiteness property.

Proof. The equivalence (i) $\Leftrightarrow$ (ii) is immediate. To prove (ii) $\Leftrightarrow$ (iii), consider an epimorphism $v: G \rightarrow A$, and let $\bar{v}=\pi \circ v: G \rightarrow \bar{A}$. We know from (2.10) that $q^{-1}([\bar{\nu}])$ coincides with the set of equivalence classes of regular $\operatorname{Tors}(A)$-covers $X^{v} \rightarrow X^{\bar{v}}$. The desired conclusion follows.

In other words, (4.7) is a pull-back diagram if and only if the homological finiteness of an arbitrary Abelian cover of $X$ can be tested through the corresponding free Abelian cover.

\section{Pontryagin duality}

Following the approach from $[9,15]$, we now discuss a functorial correspondence between finitely generated Abelian groups and Abelian, complex algebraic reductive groups. 


\subsection{A functorial correspondence}

Let $\mathbb{C}^{*}$ be the multiplicative group of units in the field of complex numbers. Given a group $G$, let $\widehat{G}=\operatorname{Hom}\left(G, \mathbb{C}^{*}\right)$ be the group of complex-valued characters of $G$, with pointwise multiplication inherited from $\mathbb{C}^{*}$, and identity the character taking constant value $1 \in \mathbb{C}^{*}$ for all $g \in G$. If the group $G$ is finitely generated, then $\widehat{G}$ is an Abelian, complex reductive algebraic group. Given a homomorphism $\varphi: G_{1} \rightarrow$ $G_{2}$, let $\hat{\varphi}: \widehat{G}_{2} \rightarrow \widehat{G}_{1}, \rho \mapsto \rho \circ \varphi$ be the induced morphism between character groups. Since the group $\mathbb{C}^{*}$ is divisible, the functor $G \rightsquigarrow \widehat{G}=\operatorname{Hom}\left(H, \mathbb{C}^{*}\right)$ is exact.

Now let $H=G_{\mathrm{ab}}$ be the maximal Abelian quotient of $G$. The Abelianization map, ab: $G \rightarrow H$, induces an isomorphism $\widehat{\mathrm{ab}}: \widehat{H} \stackrel{\simeq}{\rightrightarrows} \widehat{G}$. If $H$ is torsion-free, then $\widehat{H}$ can be identified with the complex algebraic torus $\left(\mathbb{C}^{*}\right)^{n}$, where $n=\operatorname{rank}(H)$. If $H$ is a finite Abelian group, then $\widehat{H}$ is, in fact, isomorphic to $H$.

More generally, let $\bar{H}$ be the maximal torsion-free quotient of $H$. Fixing a splitting $\bar{H} \rightarrow H$ yields a decomposition $H \cong \bar{H} \oplus \operatorname{Tors}(H)$, and thus an isomorphism $\widehat{H} \cong \widehat{\bar{H}} \times \operatorname{Tors}(H)$. For simplicity, write $T=\widehat{H}$, and $T_{0}$ for the identity component of this Abelian, reductive, complex algebraic group; clearly, $T_{0}=\widehat{\bar{H}}$ is an algebraic torus.

Conversely, we can associate to $T$ its weight group, $\check{T}=\operatorname{Hom}_{\mathrm{alg}}\left(T, \mathbb{C}^{*}\right)$, where the hom set is taken in the category of algebraic groups. The (discrete) group $\check{T}$ is a finitely generated Abelian group. Let $\mathbb{C}[\check{T}]$ be its group algebra. We then have natural identifications,

$$
\max \operatorname{Spec}(\mathbb{C}[\check{T}])=\operatorname{Hom}_{\text {alg }}(\mathbb{C}[\check{T}], \mathbb{C})=\operatorname{Hom}_{\text {group }}\left(\check{T}, \mathbb{C}^{*}\right)=T .
$$

The correspondence $H \leadsto T$ extends to a duality

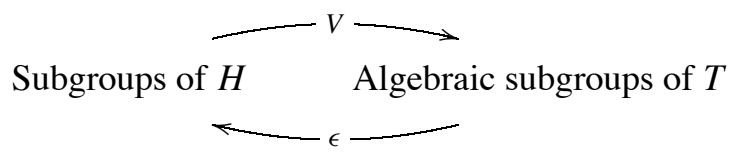

where $V$ sends a subgroup $\xi \leq H$ to $\operatorname{Hom}\left(H / \xi, \mathbb{C}^{*}\right) \subseteq T$, while $\epsilon$ sends an algebraic subgroup $C \subseteq T$ to $\operatorname{ker}(\breve{T} \rightarrow \check{C}) \leq H$.

Both sides of (5.2) are partially ordered sets, with naturally defined meets and joins. As showed in [15], the above correspondence is an order-reversing equivalence of lattices.

\subsection{Primitive lattices and connected subgroups}

Given a subgroup $\xi \leq H$, set

$$
\bar{\xi}:=\{x \in H \mid m x \in \xi \text { for some } m \in \mathbb{N}\} .
$$


Clearly, $\bar{\xi}$ is again a subgroup of $H$, and $H / \bar{\xi}$ is torsion-free. By definition, $\xi$ is a finite-index subgroup of $\bar{\xi}$; in particular, $\operatorname{rank}(\xi)=\operatorname{rank}(\bar{\xi})$. The quotient group, $\bar{\xi} / \xi$, called the determinant group of $\xi$, fits into the exact sequence

$$
0 \longrightarrow H / \bar{\xi} \longrightarrow H / \xi \longrightarrow \bar{\xi} / \xi \longrightarrow 0
$$

The inclusion $\bar{\xi} \hookrightarrow H$ induces a splitting $\bar{\xi} / \xi \hookrightarrow H / \xi$, showing that $\bar{\xi} / \xi \cong$ $\operatorname{Tors}(H / \xi)$. Since the (Abelian) group $\bar{\xi} / \xi$ is finite, it is isomorphic to its character group, $\widehat{\xi} / \xi$, which in turn can be viewed as a (finite) subgroup of $\widehat{H}=T$.

The subgroup $\xi$ is called primitive if $\bar{\xi}=\xi$. Under the correspondence $H$ ur $T$, primitive subgroups of $H$ correspond to connected algebraic subgroups of $T$. For an arbitrary subgroup $\xi \leq H$, we have an isomorphism of algebraic groups,

$$
V(\xi) \cong \widehat{\xi / \xi} \cdot V(\bar{\xi})
$$

In particular, the irreducible components of $V(\xi)$ are indexed by the determinant group, $\bar{\xi} / \xi$, while the identity component is $V(\bar{\xi})$.

\subsection{Pulling back algebraic subgroups}

Now let $v: H \rightarrow A$ be an epimorphism, and let $\bar{v}: \bar{H} \rightarrow \bar{A}$ be the induced epimorphism between maximal torsion-free quotients. Applying the $\operatorname{Hom}\left(-, \mathbb{C}^{*}\right)$ functor to the left square of (5.6) yields the commuting right square in the display below:
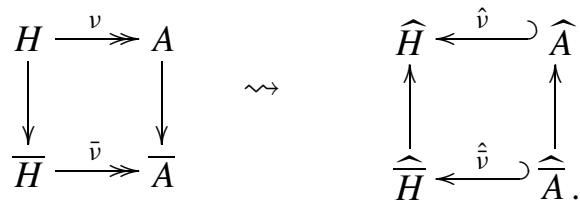

The morphism $\hat{v}: \widehat{A} \rightarrow \widehat{H}$ sends the identity component $\widehat{A}_{0}$ to the identity component $\widehat{H}_{0}$, thereby defining a morphism $\hat{v}_{0}: \widehat{A}_{0} \rightarrow \widehat{H}_{0}$. Fixing a splitting $\bar{A} \rightarrow A$ yields an isomorphism $\widehat{A} \cong \widehat{\bar{A}} \times \operatorname{Tors}(A)$. The following lemma is now clear:

Lemma 5.1. Let $v: H \rightarrow A$ be an epimorphism. Upon identifying $\widehat{\bar{A}}=\widehat{A}_{0}$ and $\widehat{\bar{H}}=\widehat{H}_{0}$, we have:

(i) $\hat{\bar{v}}=\hat{v}_{0}$,

(ii) $\operatorname{im}(\hat{v})=V(\operatorname{ker}(v))$.

Consequently, $\operatorname{im}(\hat{\bar{v}})=V(\operatorname{ker}(v))_{0}$. 


\subsection{Intersections of translated subgroups}

Before proceeding, we need to recall some results from [15], which build on work of Hironaka [9]. In what follows, $T$ will be an Abelian, reductive complex algebraic group.

Proposition 5.2 ( [15]). Let $\xi_{1}$ and $\xi_{2}$ be two subgroups of $H$, and let $\eta_{1}$ and $\eta_{2}$ be two elements in $T=\widehat{H}$. Then $\eta_{1} V\left(\xi_{1}\right) \cap \eta_{2} V\left(\xi_{2}\right) \neq \emptyset$ if and only if $\eta_{1} \eta_{2}^{-1} \in$ $V\left(\xi_{1} \cap \xi_{2}\right)$, in which case

$$
\operatorname{dim} \eta_{1} V\left(\xi_{1}\right) \cap \eta_{2} V\left(\xi_{2}\right)=\operatorname{rank} H-\operatorname{rank}\left(\xi_{1}+\xi_{2}\right) .
$$

Proposition 5.3 ( [15]). Let $C$ and $V$ be two algebraic subgroups of $T$.

(i) Suppose $\alpha_{1}, \alpha_{2}$, and $\eta$ are torsion elements in $T$ such that $\alpha_{i} C \cap \eta V \neq \emptyset$, for $i=1,2$. Then

$$
\operatorname{dim}\left(\alpha_{1} C \cap \eta V\right)=\operatorname{dim}\left(\alpha_{2} C \cap \eta V\right) .
$$

(ii) Suppose $\alpha$ and $\eta$ are torsion elements in $T$, of coprime order. Then

$$
C \cap \eta V=\emptyset \Longrightarrow \alpha C \cap \eta V=\emptyset .
$$

Here is a corollary, which will be useful later on:

Corollary 5.4. Let $C$ and $V$ be two algebraic subgroups of $T$. Suppose $\alpha$ and $\rho$ are torsion elements in $T$, such that $\rho \notin C V, \alpha^{-1} \rho \in C V$, and $\operatorname{dim}(C \cap V)>0$. Then $C \cap \rho V=\emptyset$ and $\operatorname{dim}(\alpha C \cap \rho V)>0$.

Proof. By Proposition 5.2,

$$
\rho \notin C \cdot V \Leftrightarrow C \cap \rho V=\emptyset \text { and } \alpha^{-1} \rho \in C \cdot V \Leftrightarrow \alpha C \cap \rho V \neq \emptyset .
$$

By Proposition 5.3, $\operatorname{dim}(\alpha C \cap \rho V)=\operatorname{dim}(C \cap V)>0$. The conclusion follows.

\section{An algebraic analogue of the exponential tangent cone}

We now associate to each subvariety $W \subset T$ and integer $d \geq 1$ a finite collection, $\Xi_{d}(W)$, of subgroups of the weight group $H=\check{T}$, which allows us to generalize the exponential tangent cone construction from [4].

\subsection{A collection of subgroups}

Let $T$ be an Abelian, reductive, complex algebraic group, and consider a Zariski closed subset $W \subset T$. The translated subtori contained in $W$ define an interesting collection of subgroups of the discrete group $H=\check{T}$. 
Definition 6.1. Given a subvariety $W \subset T$, and a positive integer $d$, let $\Xi_{d}(W)$ be the collection of all subgroups $\xi \leq H$ for which the following two conditions are satisfied:

(i) The determinant group $\bar{\xi} / \xi$ is cyclic of order dividing $d$;

(ii) There is a generator $\eta \in \widehat{\xi / \xi}$ such that $\eta \cdot V(\bar{\xi})$ is a maximal, positivedimensional, torsion-translated subtorus in $W$.

Clearly, if $d \mid m$, then $\Xi_{d}(W) \subseteq \Xi_{m}(W)$. Although this is not a priori clear from the definition, we shall see in Proposition 6.8 that $\Xi_{d}(W)$ is finite, for each $d \geq 1$.

To gain more insight into this concept, let us work out what the sets $\Xi_{d}(W)$ look like in the case when $W$ is a coset of an algebraic subgroup of $T$.

Lemma 6.2. Suppose $W=\eta V(\chi)$, where $\chi$ is a subgroup of $H$ and $\eta \in \widehat{H}$ is a torsion element. Write $V(\chi)=\bigcup_{\rho \in \widehat{\bar{\chi} / \chi}} \rho V(\bar{\chi})$. Then

$$
\Xi_{d}(W)=\left\{\xi \leq H \mid \exists \rho \in \widehat{\chi} \widehat{\chi \chi} \text { such that } \operatorname{ord}(\eta \rho) \mid d \text { and } \widehat{H / \xi}=\bigcup_{m \geq 1}(\eta \rho)^{m} V(\bar{\chi})\right\} \text {. }
$$

Corollary 6.3. If $\chi$ is a primitive subgroup of $H$ and $\eta$ is an element of order $d$ in $\widehat{H}$, then $\Xi_{d}(\eta V(\chi))$ consists of the single subgroup $\xi \leq \chi$ for which $\bar{\xi}=\chi$ and $\widehat{\chi / \xi}=\langle\eta\rangle$.

Now note that $\Xi_{d}$ commutes with unions: if $W_{1}$ and $W_{2}$ are two subvarieties of $T$, then

$$
\Xi_{d}\left(W_{1} \cup W_{2}\right)=\Xi_{d}\left(W_{1}\right) \cup \Xi_{d}\left(W_{2}\right) .
$$

Lemma 6.2, then, provides an algorithm for computing the sets $\Xi_{d}(W)$, whenever $W$ is a (finite) union of torsion-translated algebraic subgroups of $T$.

Example 6.4. Let $H=\mathbb{Z}^{2}$, and consider the subvariety $W=\left\{(t, 1) \mid t \in \mathbb{C}^{*}\right\} \cup$ $\left\{(-1, t) \mid t \in \mathbb{C}^{*}\right\}$ inside $T=\left(\mathbb{C}^{*}\right)^{2}$. Note that $W=V\left(\xi_{1}\right) \cup \eta V\left(\xi_{2}\right)$, where $\xi_{1}=0 \oplus \mathbb{Z}, \xi_{2}=\mathbb{Z} \oplus 0$, and $\eta=(-1,1)$. Hence, $\Xi_{d}(W)=\left\{\xi_{1}\right\}$ if $d$ is odd, and $\Xi_{d}(W)=\left\{\xi_{1}, 2 \xi_{2}\right\}$ if $d$ is even.

\subsection{The exponential map}

Consider now the lattice

$$
\mathcal{H}=H^{\vee}:=\operatorname{Hom}(H, \mathbb{Z}) .
$$

Evidently, $\mathcal{H} \cong H / \operatorname{Tors}(H)$. Moreover, each subgroup $\xi \leq H$ gives rise to a sublattice $(H / \xi)^{\vee} \leq H^{\vee}$.

Let $\operatorname{Lie}(T)$ be the Lie algebra of the complex algebraic group $T$. The exponential map exp: $\operatorname{Lie}(T) \rightarrow T$ is an analytic map, whose image is $T_{0}$. Let us identify $T_{0}=\operatorname{Hom}\left(\mathcal{H}^{\vee}, \mathbb{C}^{*}\right)$ and $\operatorname{Lie}(T)=\operatorname{Hom}\left(\mathcal{H}^{\vee}, \mathbb{C}\right)$. Under these identifications, the corestriction to the image of the exponential map can be written as

$$
\exp =\operatorname{Hom}\left(-, e^{2 \pi \mathrm{i} z}\right): \operatorname{Hom}\left(\mathcal{H}^{\vee}, \mathbb{C}\right) \rightarrow \operatorname{Hom}\left(\mathcal{H}^{\vee}, \mathbb{C}^{*}\right),
$$


where $z \mapsto e^{z}$ is the usual exponential map from $\mathbb{C}$ to $\mathbb{C}^{*}$. Finally, upon identifying $\operatorname{Hom}\left(\mathcal{H}^{\vee}, \mathbb{C}\right)$ with $\mathcal{H} \otimes \mathbb{C}$, we see that $T_{0}=\exp (\mathcal{H} \otimes \mathbb{C})$

The correspondence $T \rightsquigarrow \mathcal{H}=(\check{T})^{\vee}$ sends an algebraic subgroup $W$ inside $T$ to the sublattice $\chi=(\breve{W})^{\vee}$ inside $\mathcal{H}$. Clearly, $\chi=\operatorname{Lie}(W) \cap \mathcal{H}$ is a primitive lattice; furthermore, $\exp (\chi \otimes \mathbb{C})=W_{0}$. As shown in [15], we have

$$
V\left((\mathcal{H} / \chi)^{\vee}\right)=\exp (\chi \otimes \mathbb{C})
$$

where both sides are connected algebraic subgroups inside $T_{0}=\exp (\mathcal{H} \otimes \mathbb{C})$.

\subsection{Exponential interpretation}

The construction from Section 6.1 allows us to associate to each subvariety $W \subset T$ and each integer $d \geq 1$ a subset $\tau_{d}(W) \subseteq H^{\vee}$, given by

$$
\tau_{d}(W)=\bigcup_{\xi \in \Xi_{d}(W)}(H / \xi)^{\vee} .
$$

The next lemma reinterprets the set $\tau_{1}(W)$ in terms of the "exponential tangent cone" construction introduced in [4] and studied in detail in [14].

Lemma 6.5. For every subvariety $W \subset T$,

$$
\tau_{1}(W)=\left\{x \in H^{\vee} \mid \exp (\lambda x) \in W, \text { for all } \lambda \in \mathbb{C}\right\}
$$

Proof. Denote by $\tau$ the right-hand side of (6.6). Given a non-zero homomorphism $x: H \rightarrow \mathbb{Z}$ such that $x \in \tau$, the subgroup $\operatorname{ker}(x) \leq H$ is primitive and $V(\operatorname{ker}(x))=$ $\exp (\mathbb{C} x) \subseteq W$. Hence, we can find a subgroup $\xi \leq H$ such that $\xi$ is primitive, $V(\operatorname{ker}(x)) \subseteq V(\xi)$, and $V(\xi) \subseteq W$ is a maximal subtorus. By (6.4), we have that $V(\xi)=\exp \left((H / \xi)^{\vee} \otimes \mathbb{C}\right)$, which implies $x \in \tau_{1}(W)$.

Conversely, for any non-zero element $x \in \tau_{1}(W)$, there is a subgroup $\xi \leq$ $H$ such that $\xi \in \Xi_{1}(W)$ and $x \in(H / \xi)^{\vee}$. Thus, $\mathbb{C} x \subseteq(H / \xi)^{\vee} \otimes \mathbb{C}$, and so $\exp (\mathbb{C} x) \subseteq \exp \left((H / \xi)^{\vee} \otimes \mathbb{C}\right)=V(\xi) \subseteq W$. Since $x \neq 0$, the map $x: H \rightarrow \mathbb{Z}$ is surjective; thus, $V(\operatorname{ker}(x))=V\left((\mathcal{H} / \chi)^{\vee}\right)$, where $\chi$ is the rank-1 sublattice of $\mathcal{H}$ generated by $x$. Hence, $V(\operatorname{ker}(x))=\exp (\chi \otimes \mathbb{C}) \subseteq W$, and so $x \in \tau$.

Using now the characterization of exponential tangent cones given in $[4,14]$, we obtain the following immediate:

Corollary 6.6. $\tau_{1}(W)$ is a finite union of subgroups of $H^{\vee}$.

Thus, the set $\tau_{1}^{\mathbb{Q}}(W)=\bigcup_{\xi \in \Xi_{1}(W)}(H / \xi)^{\vee} \otimes \mathbb{Q}$ is a finite union of linear subspaces in the vector space $\mathbb{Q}^{n}$, where $n=\operatorname{rank} H$. 
Example 6.7. Let $T=\left(\mathbb{C}^{*}\right)^{n}$, and suppose $W=Z(f)$, for some Laurent polynomial $f$ in $n$ variables. Write $f\left(t_{1}, \ldots, t_{n}\right)=\sum_{a \in S} c_{a} t_{1}^{a_{1}} \cdots t_{n}^{a_{n}}$, where $S$ is a finite subset of $\mathbb{Z}^{n}$, and $c_{a} \in \mathbb{C}^{*}$ for each $a=\left(a_{1}, \ldots, a_{n}\right) \in S$. We say a partition $\mathrm{p}=\left(\mathrm{p}_{1}|\cdots| \mathrm{p}_{q}\right)$ of the support $S$ is admissible if $\sum_{a \in \mathrm{p}_{j}} c_{a}=0$, for each $1 \leq j \leq q$. To such a partition, we associate the subgroup

$$
L(\mathrm{p})=\left\{x \in \mathbb{Z}^{n} \mid(a-b) \cdot x=0, \forall a, b \in \mathrm{p}_{j}, \forall 1 \leq j \leq q\right\} .
$$

Then $\tau_{1}(W)$ is the union of all subgroups $L(\mathrm{p})$, where $\mathrm{p}$ runs through the set of admissible partitions of $S$. In particular, if $f(1) \neq 0$, then $\tau_{1}(W)=\emptyset$.

Proposition 6.8. For each $d \geq 1$, the set $\Xi_{d}(W)$ is finite.

Proof. Fix an integer $d \geq 1$. For any torsion point $\eta \in T$ whose order divides $d$, consider the set $\Xi_{d}(W, \eta)$ of subgroups $\xi \leq H$ for which $\widehat{\xi / \xi}=\langle\eta\rangle$ and $\eta \cdot V(\bar{\xi})$ is a maximal, positive-dimensional, torsion-translated subtorus in $W$. Then

$$
\Xi_{d}(W)=\bigcup_{\eta} \Xi_{d}(W, \eta)
$$

where the union runs over the (finite) set of torsion points $\eta \in T$ whose order divides $d$.

For each such point $\eta$, we have a map $\Xi_{d}(W, \eta) \rightarrow \Xi_{1}\left(\eta^{-1} W\right), \xi \mapsto \bar{\xi}$. Clearly, this map is an injection. Now, Corollary 6.6 insures that the set $\Xi_{1}(W)$ is finite. Thus, the set $\Xi_{1}\left(\eta^{-1} W\right)$ is also finite, and we are done.

\section{The incidence correspondence for subgroups of $H$}

We now single out certain subsets $\sigma_{A}(\xi)$ and $U_{A}(\xi)$ of the parameter set $\Gamma(H, A)$, which may be viewed as analogues of the special Schubert varieties in Grassmann geometry.

\subsection{The sets $\sigma_{A}(\xi)$}

We start by recalling a classical geometric construction. Let $V$ be a variety in $\mathbb{Q}^{n}$ defined by homogeneous polynomials. Set $m=\operatorname{dim} V$, and assume $m>0$. Consider the locus of $r$-planes in $\mathbb{Q}^{n}$ intersecting $V$ non-trivially,

$$
\sigma_{r}(V)=\left\{P \in \mathrm{Gr}_{r}\left(\mathbb{Q}^{n}\right) \mid \operatorname{dim}(P \cap V)>0\right\} .
$$

This set is a Zariski closed subset of $\operatorname{Gr}_{r}\left(\mathbb{Q}^{n}\right)$, called the variety of incident $r$ planes to $V$. For all $0<r<n-m$, this is an irreducible subvariety, of dimension $(r-1)(n-r)+m-1$.

Particularly simple is the case when $V$ is a linear subspace $L \subset \mathbb{Q}^{n}$. The corresponding incidence variety, $\sigma_{r}(L)$, is known as the special Schubert variety 
defined by $L$. Clearly, $\sigma_{1}(L)=\mathbb{P}(L)$, viewed as a projective subspace in $\mathbb{Q P}^{n-1}:=$ $\mathbb{P}\left(\mathbb{Q}^{n}\right)$.

Now let $H$ be a finitely generated Abelian group, let $A$ be a factor group of $H$, and let $\Gamma(H, A)=\operatorname{Epi}(H, A) / \operatorname{Aut}(A)$.

Definition 7.1. Given a subgroup $\xi \leq H$, let $\sigma_{A}(\xi)$ be the set of all $[v] \in \Gamma(H, A)$ for which $\operatorname{rank}(\operatorname{ker}(v)+\xi)<\operatorname{rank} H$.

When $A$ is torsion-free, we recover the classical definition of special Schubert varieties. More precisely, set $n=\operatorname{rank} H$ and $r=\operatorname{rank} A$. We then have the following lemma.

Lemma 7.2. Under the natural isomorphism $\Gamma(H, \bar{A}) \cong \mathrm{Gr}_{r}\left(\mathbb{Q}^{n}\right)$, the set $\sigma_{\bar{A}}(\xi)$ corresponds to the special Schubert variety $\sigma_{r}\left((H / \xi)^{\vee} \otimes \mathbb{Q}\right)$.

Proof. Let $\mathrm{Gr}_{r}\left(H^{\vee} \otimes \mathbb{Q}\right)$ be the Grassmannian of $r$-dimensional subspaces in the vector space $H^{\vee} \otimes \mathbb{Q} \cong \mathbb{Q}^{n}$. Given an epimorphism $v: H \rightarrow \bar{A}$ and a subgroup $\xi \leq H$, we have

$$
\operatorname{rank}(\operatorname{ker}(v)+\xi)<\operatorname{rank} H \Longleftrightarrow \operatorname{dim}\left((H / \operatorname{ker}(v))^{\vee} \otimes \mathbb{Q} \cap(H / \xi)^{\vee} \otimes \mathbb{Q}\right)>0 .
$$

Thus, the isomorphism

$$
\Gamma(H, \bar{A}) \stackrel{\simeq}{\lessgtr} \operatorname{Gr}_{r}\left(H^{\vee} \otimes \mathbb{Q}\right), \quad[\nu] \mapsto(H / \operatorname{ker}(v))^{\vee} \otimes \mathbb{Q}
$$

establishes a one-to-one correspondence between $\sigma_{\bar{A}}(\xi)$ and $\sigma_{r}\left((H / \xi)^{\vee} \otimes \mathbb{Q}\right)$.

For instance, if $A$ is infinite cyclic, then the parameter set $\Gamma(H, \mathbb{Z})$ may be identified with the projective space $\mathbb{P}\left(H^{\vee}\right)$, while the set $\sigma_{\mathbb{Z}}(\xi)$ coincides with the projective subspace $\mathbb{P}\left((H / \xi)^{\vee}\right)$.

Example 7.3. Let $\xi \leq \mathbb{Z}^{2}$ be the sublattice spanned by the vector $(a, b) \in \mathbb{Z}^{2}$. Then $\sigma_{\mathbb{Z}}(\xi) \subset \Gamma\left(\mathbb{Z}^{2}, \mathbb{Z}\right)$ corresponds to the point $(-b, a) \in \mathbb{Q P}^{1}$.

The sets $\sigma_{A}(\xi)$ can be reconstructed from the classical Schubert varieties $\sigma_{\bar{A}}(\xi)$ associated to the lattice $\bar{A}=A$ / Tors $(A)$ by means of the set fibration described in Theorem 3.2. More precisely, we have the following:

Proposition 7.4. Let $q: \Gamma(H, A) \rightarrow \Gamma(H, \bar{A})$ be the natural projection map. Then

(i) $q\left(\sigma_{A}(\xi)\right)=\sigma_{\bar{A}}(\xi)$,

(ii) $q^{-1}\left(\sigma_{\bar{A}}(\xi)\right)=\sigma_{A}(\xi)$.

Therefore, $\sigma_{A}(\xi)$ fibers over the Schubert variety $\sigma_{\bar{A}}(\xi)$, with each fiber isomorphic to the set $\Gamma(H / \bar{A}, A / \bar{A})$. 


\subsection{The sets $U_{A}(\xi)$}

Although simple to describe, the sets $\sigma_{A}(\xi)$ do not behave too well with respect to the correspondence between subgroups of $H$ and algebraic subgroups of $T=\widehat{H}$. This is mainly due to the fact that the $\sigma_{A}$-sets do not distinguish between a subgroup $\xi \leq H$ and its primitive closure, $\bar{\xi}$. To remedy this situation, we identify certain subsets $U_{A}(\xi) \subseteq \sigma_{A}(\xi)$ which turn out to be better suited for our purposes.

Definition 7.5. Given a subgroup $\xi \leq H$, let $U_{A}(\xi)$ be the set of all $[\nu] \in \sigma_{A}(\xi)$ for which $\operatorname{ker}(v) \cap \bar{\xi} \subseteq \xi$.

In particular, if $\bar{\xi}=\xi$, then $U_{A}(\xi)=\sigma_{A}(\xi)$. In general, though, $U_{A}(\xi) \varsubsetneqq$ $\sigma_{A}(\xi)$. In order to reinterpret this definition in more geometric terms, we need a lemma.

Lemma 7.6. Let $\xi \leq H$ be a subgroup such that $\bar{\xi} / \xi$ is cyclic, and let $\chi \leq H$ be another a subgroup. Then the following conditions are equivalent:

(i) $\chi \cap \bar{\xi} \subseteq \xi$,

(ii) $V(\chi) \cap \eta V(\bar{\xi}) \neq \emptyset$, for some generator $\eta$ of $\widehat{\xi / \xi}$,

(iii) $V(\chi) \cap \eta V(\bar{\xi}) \neq \emptyset$, for any generator $\eta$ of $\bar{\xi} / \xi$,

(iv) $\eta \in V(\operatorname{ker}(v) \cap \bar{\xi})$, for some generator $\eta$ of $\overline{\xi / \xi}$,

(v) $\eta \in V(\operatorname{ker}(v) \cap \bar{\xi})$, for any generator $\eta$ of $\overline{\xi / \xi}$.

Proof. Let $\eta$ be a generator of the finite cyclic group $\widehat{\xi / \xi}$. We then have

$$
\epsilon(\langle\eta\rangle) \cap \bar{\xi}=\xi .
$$

By Proposition 5.2, the intersection $V(\chi) \cap \eta V(\bar{\xi})$ is non-empty if and only if $\eta \in V(\chi \cap \bar{\xi})$, that is, $\langle\eta\rangle \subseteq V(\chi \cap \bar{\xi})$, which in turn is equivalent to

$$
\epsilon(\langle\eta\rangle) \supseteq \chi \cap \bar{\xi} .
$$

In view of equality (7.2), inclusion (7.3) is equivalent to $\chi \cap \bar{\xi} \subseteq \xi$. This shows (i) $\Leftrightarrow$ (ii).

The other equivalences are proved similarly.

Corollary 7.7. Let $\xi \leq H$ be a subgroup, and assume $\bar{\xi} / \xi$ is cyclic. Let $v: H \rightarrow A$ be an epimorphism. Then

$$
[v] \in U_{A}(\xi) \Longleftrightarrow \operatorname{dim}(V(\operatorname{ker}(v)) \cap \eta V(\bar{\xi}))>0
$$

for any (or, equivalently, for some) generator $\eta \in \widehat{\xi / \xi}$.

Despite their geometric appeal, the sets $U_{A}(\xi)$ do not enjoy a naturality property analogous to the one from Proposition 7.4. In particular, the projection map $q: \Gamma(H, A) \rightarrow \Gamma(H, \bar{A})$ may not restrict to a map $U_{A}(\xi) \rightarrow U_{\bar{A}}(\xi)$. Here is a simple example:

Example 7.8. Let $v: H \rightarrow A$ be the canonical projection from $H=\mathbb{Z}^{2}$ to $A=$ $\mathbb{Z} \oplus \mathbb{Z}_{2}$, and let $\xi=\operatorname{ker}(v)$. Then $[v] \in U_{A}(\xi)$, but $[\bar{v}] \notin U_{\bar{A}}(\xi)$. 


\section{The incidence correspondence for subvarieties of $\widehat{H}$}

In this section, we introduce and study certain subsets $\Upsilon_{A}(W) \subseteq \Gamma(H, A)$, which can be viewed as the toric analogues of the classical incidence varieties $\sigma_{r}(V) \subseteq$ $\operatorname{Gr}_{r}\left(\mathbb{Q}^{n}\right)$.

\subsection{The sets $U_{A}(W)$}

Let us start by recalling some constructions we discussed previously. In Section 6.1, we associated to each subvariety $W$ of the algebraic group $T=\widehat{H}$ and each integer $d \geq 1$ a certain collection $\Xi_{d}(W)$ of subgroups of $H$. In Section 7.2, we associated to each subgroup $\xi \leq H$ and each Abelian group $A$ a certain subset $U_{A}(\xi)$ of the parameter set $\Gamma(H, A)=\operatorname{Epi}(H, A) / \operatorname{Aut}(A)$. Putting together these two constructions, we associate now to $W$ a family of subsets of $\Gamma(H, A)$, as follows.

Definition 8.1. Given a subvariety $W \subset T$, an Abelian group $A$, let

$$
U_{A}(W)=\bigcup_{d \geq 1} U_{A, d}(W),
$$

where

$$
U_{A, d}(W)=\bigcup_{\xi \in \Xi_{d}(W)} U_{A}(\xi) .
$$

By Proposition 6.8, the union in (8.2) is a finite one.

Lemma 8.2. The set $U_{A, d}(W)$ consists of all $[\nu] \in \Gamma(H, A)$ for which there is a subgroup $\xi \leq H$ and an element $\eta \in \widehat{H}$ of order dividing $d$ such that $\eta V(\bar{\xi})$ is a maximal, positive-dimensional translated subtorus in $W$, and $\operatorname{dim}(V(\operatorname{ker} v) \cap$ $\eta V(\bar{\xi}))>0$.

Proof. Let $v: H \rightarrow A$ be an epimorphism such that $[v] \in U_{A}(\xi)$, for some $\xi \in$ $\Xi_{d}(W)$. According to Definition 6.1 , this means that the group $\bar{\xi} / \xi$ is cyclic of order dividing $d$, and there is a generator $\eta \in \widehat{\xi / \xi}$ such that $\eta V(\bar{\xi})$ is a maximal, positive-dimensional translated subtorus in $W$. In view of Corollary 7.7, the fact that $[v] \in U_{A}(\xi)$ insures that $V(\operatorname{ker}(v)) \cap \eta V(\bar{\xi})$ has positive dimension.

The case $d=1$ is worth singling out:

Corollary 8.3. Let $W \subset T$ be a subvariety. Set $n=\operatorname{rank} H$ and $r=\operatorname{rank} A$. Then:

(i) $U_{A, 1}(W)=\bigcup_{\xi \in \Xi_{1}(W)} \sigma_{A}(\xi)$;

(ii) Under the isomorphism $\Gamma(H, \bar{A}) \cong \mathrm{Gr}_{r}\left(\mathbb{Q}^{n}\right)$, the set $U_{\bar{A}, 1}(W)$ corresponds to the incidence variety $\sigma_{r}\left(\tau_{1}^{\mathbb{Q}}(W)\right)$.

In general, the set $U_{A}(W)$ is larger than $U_{A, 1}(W)$. Here is a simple example; a more general situation will be studied in Section 12.3.

Example 8.4. Let $H=\mathbb{Z}^{2}$ and let $W \subset\left(\mathbb{C}^{*}\right)^{2}$ be the subvariety from Example 6.4. Pick $A=\mathbb{Z} \oplus \mathbb{Z}_{2}$, and identify $\Gamma(H, A)$ with $\mathbb{Q P}^{1}$. Then $U_{A, d}(W)=\{(1,0)\}$ or $\{(1,0),(0,1)\}$, according to whether $d$ is odd or even. 


\subsection{The sets $\Upsilon_{A}(W)$}

As before, let $H$ be a finitely-generated Abelian group, and let $T=\widehat{H}$ be its Pontryagin dual. The next definition will prove to be key to the geometric interpretation of the (generalized) Dwyer-Fried invariants.

Definition 8.5. Given a subvariety $W \subset T$, and an Abelian group $A$, define a subset $\Upsilon_{A}(W)$ of the parameter set $\Gamma(H, A)$ by setting

$$
\Upsilon_{A}(W)=\{[\nu] \in \Gamma(H, A) \mid \operatorname{dim}(V(\operatorname{ker} v) \cap W)>0\} .
$$

Roughy speaking, the set $\Upsilon_{A}(W) \subset \Gamma(H, A)$ associated to a variety $W \subset T$ is the toric analogue of the incidence variety $\sigma_{r}(V) \subset \mathrm{Gr}_{r}\left(\mathbb{Q}^{n}\right)$ associated to a homogeneous variety $V \subset \mathbb{Q}^{n}$.

It is readily seen that $\Upsilon_{A}$ commutes with unions: if $W_{1}$ and $W_{2}$ are two subvarieties of $T$, then

$$
\Upsilon_{A}\left(W_{1} \cup W_{2}\right)=\Upsilon_{A}\left(W_{1}\right) \cup \Upsilon_{A}\left(W_{2}\right) .
$$

Moreover, $\Upsilon_{A}(W)$ depends only on the positive-dimensional components of $W$. Indeed, if $Z$ is a finite algebraic set, then $\Upsilon_{A}(W \cup Z)=\Upsilon_{A}(W)$.

The next result gives a convenient lower bound for the $\Upsilon$-sets.

Proposition 8.6. Let A be a quotient of $H$. Then

$$
U_{A}(W) \subseteq \Upsilon_{A}(W) .
$$

Proof. Let $v: H \rightarrow A$ be an epimorphism such that $[v] \in U_{A, d}(W)$, for some $d \geq$ 1. By Lemma 8.2, we have that $\operatorname{dim}(V(\operatorname{ker} v) \cap W)>0$. Thus, $[v] \in \Upsilon_{A}(W)$.

As we shall see in Example 10.8, inclusion (8.5) may well be strict.

\subsection{Translated subgroups}

If the variety $W$ is a torsion-translated algebraic subgroup of $T$, we can be more precise.

Theorem 8.7. Let $W=\eta V(\xi)$, where $\xi \leq H$ is a subgroup, and $\eta \in \widehat{H}$ has finite order. Then $\Upsilon_{A}(W)=U_{A, c}(W)$, where $c=\operatorname{ord}(\eta) \cdot c(\bar{\xi} / \xi)$.

Proof. Inclusion $\supseteq$ follows from Proposition 8.6, so we only need to prove the opposite inclusion. Write

$$
V(\xi)=\bigcup_{\rho \in \overline{\xi / \xi}} \rho V(\bar{\xi}) .
$$

Let $v: H \rightarrow A$ be an epimorphism such that $[v] \in \Upsilon_{A}(\eta V(\xi))$. Hence, there is a character $\rho: \bar{\xi} / \xi \rightarrow \mathbb{C}^{*}$ such that $\operatorname{dim}(V(\operatorname{ker}(v)) \cap \eta \rho V(\bar{\xi}))>0$. Consider the subgroup

$$
\chi=\epsilon\left(\bigcup_{m}(\eta \rho)^{m} V(\bar{\xi})\right) \text {. }
$$


Lemma 6.2 implies that $\chi \in \Xi_{d}(\eta V(\xi))$, where $d=\operatorname{ord}(\eta \rho)$. Using Corollary 7.7, we conclude that $[v] \in U_{A}(\chi)$. done.

Finally, set $c=\operatorname{ord}(\eta) \cdot c(\bar{\xi} / \xi)$. Then clearly $[v] \in U_{A, c}(W)$, and we are

Here is an alternate description of the set $\Upsilon_{A}(W)$, in the case when $W$ is an algebraic subgroup of $T$, translated by an element $\eta \in T$, not necessarily of finite order.

Theorem 8.8. Let $\xi \leq H$ be a subgroup, and let $\eta \in \widehat{H}$. Then

$$
\Upsilon_{A}(\eta V(\xi))=\sigma_{A}(\xi) \cap\{[v] \in \Gamma(H, A) \mid \eta \in V(\operatorname{ker}(v) \cap \xi)\} .
$$

In particular, $\Upsilon_{A}(V(\xi))=\sigma_{A}(\xi)$.

Proof. By Proposition 5.2, we have

$$
\begin{aligned}
& \{[v] \mid \operatorname{dim}(V(\operatorname{ker}(v)) \cap \eta V(\xi))>0\} \\
& \quad=\{[v] \mid V(\operatorname{ker}(v)) \cap \eta V(\xi) \neq \emptyset\} \cap\{[v] \mid \operatorname{rank}(\operatorname{ker}(v)+\xi)<\operatorname{rank} H\} .
\end{aligned}
$$

Moreover, $V(\operatorname{ker}(v)) \cap \eta V(\xi) \neq \emptyset \Longleftrightarrow \eta \in V(\operatorname{ker}(v) \cap \xi)$, and we are done.

Remark 8.9. In the case when $A$ is a free Abelian group of rank $r$ and $\xi$ is a primitive subgroup of $H=\mathbb{Z}^{n}$, the set $\Upsilon_{A}(\eta V(\xi))$ coincides with the set $\sigma_{r}(\xi, \eta)$ defined in [14].

When the translation factor $\eta$ from Theorem 8.8 has finite order, a bit more can be said:

Corollary 8.10. Let $W=\eta V(\xi)$ be a torsion-translated subgroup of $T$. Then

$$
\Upsilon_{A}(W)=\sigma_{A}(\xi) \cap\{[v] \in \Gamma(H, A) \mid \epsilon(\langle\eta\rangle) \supseteq \operatorname{ker}(v) \cap \xi\} .
$$

\subsection{Deleted subgroups}

We now analyze in more detail the case when the variety $W$ is obtained from an algebraic subgroup of $T$ by deleting its identity component. First, we need to introduce one more bit of notation.

Definition 8.11. Given a subgroup $\xi \leq H$, and a quotient $A$ of $H$, consider the subset $\theta_{A}(\xi) \subseteq \Gamma(H, A)$ given by

$$
\theta_{A}(\xi)=\bigcup_{\xi \leq \xi^{\prime} \ddagger \bar{\xi}: \bar{\xi} / \xi^{\prime} \text { is cyclic }}\left\{[v] \in \Gamma(H, A) \mid v(x) \neq 0 \text { for all } x \in \bar{\xi} \backslash \xi^{\prime}\right\} .
$$

Note that the indexing set for this union is a finite set, which is empty if $\xi$ is primitive. On the other hand, the condition that $v(x) \neq 0$ depends on the actual element $x$ in the (typically) infinite set $\bar{\xi} \backslash \xi^{\prime}$, not just on the class of $x$ in the finite group $\bar{\xi} / \xi^{\prime}$. Thus, even when $A=\mathbb{Z}^{r}$, the set $\theta_{A}(\xi)$ need not be open in the Grassmannian $\Gamma(H, A)=\operatorname{Gr}_{r}\left(\mathbb{Q}^{n}\right)$, where $n=\operatorname{rank}(H)$, although each of the sets $\{[v] \mid v(x) \neq 0\}$ is open. 
Proposition 8.12. Suppose $W=V(\xi) \backslash V(\bar{\xi})$, for some non-primitive subgroup $\xi \leq H$. Then

$$
\Upsilon_{A}(W)=\sigma_{A}(\xi) \cap \theta_{A}(\xi) .
$$

Proof. Write $W=\bigcup_{\eta \in \widehat{\xi} / \bar{\xi} \backslash\{1\}} \eta V(\bar{\xi})$. By Theorem 8.8 and Lemma 7.6, we have

$$
\begin{aligned}
\Upsilon_{A}(W) & =\Upsilon_{A}\left(\bigcup_{\eta \in \bar{\xi} / \xi \backslash\{1\}} \eta V(\bar{\xi})\right) \\
& =\bigcup_{\eta \in \widehat{\xi} / \xi \backslash\{1\}}\left(\sigma_{A}(\bar{\xi}) \cap\{[\nu] \in \Gamma(H, A) \mid \eta \in V(\operatorname{ker}(v) \cap \bar{\xi})\}\right) \\
& =\sigma_{A}(\bar{\xi}) \cap \bigcup_{\eta \in \bar{\xi} / \xi \backslash\{1\}}\{[v] \in \Gamma(H, A) \mid V(\operatorname{ker}(v)) \cap \eta V(\bar{\xi}) \neq \emptyset\} \\
& =\sigma_{A}(\xi) \cap \bigcup_{\xi \leq \xi^{\prime} \leq \bar{\xi}: \bar{\xi} / \xi^{\prime} \text { is cyclic }}\left\{[\nu] \in \Gamma(H, A) \mid \operatorname{ker}(v) \cap \bar{\xi} \leq \xi^{\prime}\right\} .
\end{aligned}
$$

The desired conclusion follows at once.

\subsection{Comparing the sets $\Upsilon_{A}(W)$ and $\Upsilon_{\bar{A}}(W)$}

Fix a decomposition $A=\bar{A} \oplus \operatorname{Tors}(A)$. Clearly, the projection map $q=q_{A}: \Gamma(H, A) \rightarrow$ $\Gamma(H, \bar{A})$ sends $\Upsilon_{A}(W)^{c}$ to $\Upsilon_{\bar{A}}(W)^{c}$. On the other hand, as we shall see in Example 10.8, the map $q$ does not always send $\Upsilon_{A}(W)$ to $\Upsilon_{\bar{A}}(W)$. Nevertheless, in some special cases it does. Here is one such situation:

Proposition 8.13. Suppose $W=\rho T$, where $T \subset \widehat{H}$ is an algebraic subgroup, and $\bar{\rho} \in \widehat{H} / T$ has finite order, coprime to the order of $\operatorname{Tors}(A)$. Then

$$
q\left(\Upsilon_{A}(W)\right)=\Upsilon_{\bar{A}}(W) \text { and } q^{-1}\left(\Upsilon_{\bar{A}}(W)\right)=\Upsilon_{A}(W) .
$$

Therefore, $\Upsilon_{A}(W)$ fibers over $\Upsilon_{\bar{A}}(W)$, with each fiber isomorphic to $\Gamma(H / \bar{A}, A / \bar{A})$.

Here, $\bar{\rho}$ is the image of $\rho$ under the quotient map $\widehat{H} \rightarrow \widehat{H} / T$.

Proof. Let $v: H \rightarrow A$ be an epimorphism such that $[\bar{v}]=q([v])$ does not belong to $\Upsilon_{\bar{A}}(W)$, that is, the subtorus $\operatorname{im}(\hat{\bar{v}})=\operatorname{im}(\hat{v})_{1}$ intersects $W$ in only finitely many points. We want to show that $\operatorname{im}(\hat{v})_{\alpha} \cap W$ is also finite, for all $\alpha \in \operatorname{Tors}(A)$.

First assume $\operatorname{im}(\hat{v})_{1} \bigcap W$ is non-empty. If $\operatorname{im}(\hat{v})_{\alpha} \bigcap W=\emptyset$, we are done. Otherwise, using Proposition 5.3(i) with $C=\operatorname{im}(\hat{v})_{1}, \alpha_{1}=1, \alpha_{2}=\alpha$ and $\eta V=\rho T$, we infer that $\operatorname{dim}\left(\operatorname{im}(\hat{v})_{\alpha} \cap \rho T\right)=\operatorname{dim}\left(\operatorname{im}(\hat{v})_{1} \cap \rho T\right)$, and the desired conclusion follows.

Now assume $\operatorname{im}(\hat{v})_{1} \cap W$ is empty. Using Proposition 5.3(ii) with $C=\operatorname{im}(\hat{v})_{1}$ and $\eta V=\rho T$, our assumption that $\operatorname{im}(\hat{v})_{1} \cap \rho T=\varnothing$ implies that $\operatorname{im}(\hat{v})_{\alpha} \bigcap \rho T=\emptyset$. Thus, the desired conclusion follows in this case, too, and we are done. 
Corollary 8.14. For every subgroup $\xi \leq H$, the set $q\left(\Upsilon_{A}(V(\xi))\right)$ is contained in $\Upsilon_{\bar{A}}(V(\xi))$.

In general, though, the projection map $q: \Gamma(H, A) \rightarrow \Gamma(H, \bar{A})$ does not restrict to a map $\Upsilon_{A}(W) \rightarrow \Upsilon_{\bar{A}}(W)$. Proposition 8.16 below describes a situation when this happens. First, we need a lemma, whose proof is similar to the proof of Proposition 4.9.

Lemma 8.15. Let $\pi: A \rightarrow B$ be an epimorphism, and let $\tilde{\pi}: \Gamma(H, A) \rightarrow \Gamma(H, B)$ be the induced homomorphism. Then

$$
q_{A}\left(\Upsilon_{A}(W)\right) \subseteq \Upsilon_{\bar{A}}(W) \Longrightarrow q_{B}\left(\Upsilon_{B}(W)\right) \subseteq \Upsilon_{\bar{B}}(W)
$$

Proposition 8.16. Let $H$ be a finitely generated, free Abelian group, and let $A$ be a quotient of $H$ such that $\operatorname{rank} A<\operatorname{rank} H$. Let $W$ be a subvariety of $\widehat{H}$ of the form $\rho T \cup Z$, where $Z$ is a finite set, $T$ is an algebraic subgroup, and $\rho$ is a torsion element whose order divides $c(A)$. Then $q\left(\Upsilon_{A}(W)\right) \notin \Upsilon_{\bar{A}}(W)$.

Proof. We need to construct an epimorphism $v: H \rightarrow A$ such that $[v] \in \Upsilon_{A}(W)$, yet $[\bar{\nu}] \notin \Upsilon_{\bar{A}}(W)$.

Step 1. First, we assume Tors $(A)$ is a cyclic group. In this case, we claim there exists a subtorus $C$ of $\widehat{H}$, and a torsion element $\alpha \in \widehat{H}$, such that ord $\alpha=$ ord $\rho$ and $\operatorname{dim}(C \cap \rho T) \leq 0$, yet $\operatorname{dim}(\alpha C \cap \rho T)>0$.

To prove the claim, set $\epsilon(\langle\rho\rangle)=L$ and $\xi=\epsilon(T)$. Since $\rho \notin T$, we have that $\xi \nsubseteq L$. Thus, there exist a sublattice $\chi$ of rank-1, such that $\chi \subseteq \xi$ and $\chi \nsubseteq L$. Set $T^{\prime}=V(\chi)$. Then $T^{\prime}$ is a codimension 1 subgroup with $T \subseteq T^{\prime} \subset \widehat{H}$, and $\rho \notin T^{\prime}$. Thus, $T_{0} \subseteq T_{0}^{\prime} \subset \widehat{H}$. Let $C$ be any dimension $r$ subtorus of $T_{0}^{\prime}$ intersecting $T$ with positive dimension. Then $\rho \notin C T \subseteq T^{\prime}$ and $\operatorname{dim}(C \cap T)>0$. Choose an element $\alpha \in \widehat{H}$ such that $\alpha^{-1} \rho=1 \in C \bar{T}$. Clearly, ord $(\alpha)=\operatorname{ord}(\rho) \mid c(A)$. Using Corollary 5.4, we conclude that $C \cap \rho T=\emptyset$ and $\operatorname{dim}(\alpha C \cap \rho T)>0$, thus finishing the proof of the claim.

Now, the algebraic subgroup $\bigcup_{k} \alpha^{k} C$ corresponds to an epimorphism $H \rightarrow$ $\bar{A} \oplus \mathbb{Z}_{d}$, where $d=\operatorname{ord}(\alpha)$. Since $H$ is torsion-free, Tors $(A)$ is cyclic, and $d$ divides $c(A)$, this epimorphism can be lifted to an epimorphism $v: H \rightarrow A$.

Step 2. For the general case, let $B$ be the cyclic subgroup of $\operatorname{Tors}(A)$ for which $|B|=c(A)$ and $\operatorname{ord}(\rho)|| B \mid$. Notice that $B$ is a direct summand of Tors $(A)$. We then have the following commuting diagram:

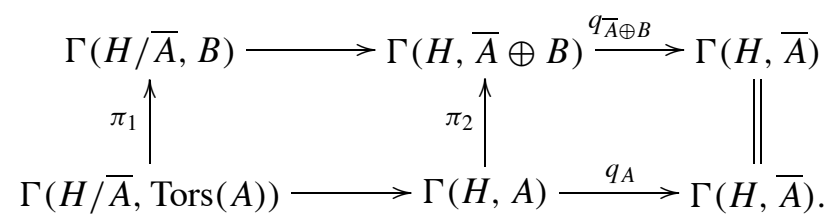

Since $H / \bar{A}$ is torsion-free, the group $\operatorname{Aut}(H / \bar{A})$ acts transitively on $\Gamma(H / \bar{A}, B)$. Using the assumption that $\Gamma(H / \bar{A}$, Tors $(A)) \neq \emptyset$, we deduce that the map $\pi_{1}$ is 
surjective. Thus, the map $\pi_{2}$ is surjective. From Step 1, we know that $q\left(\Upsilon_{\bar{A} \oplus B}(W)\right)$ is not contained in $\Upsilon_{\bar{A}}(W)$. Using Lemma 8.15, we conclude that $q\left(\Upsilon_{A}(W)\right)$ is not contained in $\Upsilon_{\bar{A}}(W)$, either.

\section{Support varieties for homology modules}

We now switch gears, and revisit the Dwyer-Fried theory in a slightly more general context. In particular, we show that the support varieties of the homology modules of two related chain complexes coincide.

\subsection{Support varieties}

Let $H$ be a finitely generated Abelian group, and let $F$ be a finitely generated $\mathbb{C}$ algebra. Then the group ring $R=F[H]$ is a Noetherian ring. Let $\max \operatorname{Spec}(R)$ be the set of maximal ideals in $R$, endowed with the Zariski topology.

Given a module $M$ over $F[H]$, denote by supp $M$ its support, consisting of those maximal ideals $\mathfrak{m} \in \max \operatorname{Spec}(F[H])$ for which the localization $M_{\mathfrak{m}}$ is nonzero.

Now let $A$ be another finitely generated Abelian group, and let $v: H \rightarrow A$ be an epimorphism. Denote by $S=F[A]$ the group ring of $A$. The extension of $v$ to group rings, $v: R \rightarrow S$, is a ring epimorphism. Let $v^{*}: \max \operatorname{Spec}(S) \hookrightarrow$ $\max \operatorname{Spec}(R)$ be the induced morphism between the corresponding affine varieties.

In the case when $F=\mathbb{C}$, the group ring $R=\mathbb{C}[H]$ is the coordinate ring of the algebraic group $\widehat{H}=\operatorname{Hom}\left(G, \mathbb{C}^{*}\right)$, and $\max \operatorname{Spec}(R)=\widehat{H}$. Furthermore, if $M$ is an $R$-module, then

$$
\operatorname{supp}(M)=Z(\operatorname{ann} M),
$$

where ann $M \subset R$ is the annihilator of $M$, and $Z(\operatorname{ann} M) \subset \widehat{H}$ is its zero-locus.

Lemma 9.1. If $v: H \rightarrow A$ is an epimorphism, then

$$
\left(v^{*}\right)^{-1}(\operatorname{supp}(M)) \cong \operatorname{im}(\hat{v}) \cap Z(\operatorname{ann} M) .
$$

Proof. From the definitions, we see that the diagram

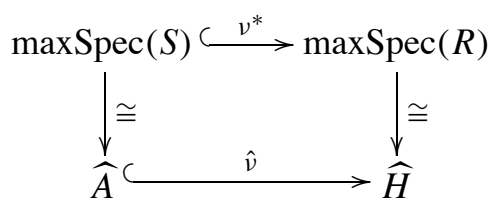

commutes. The conclusion readily follows. 


\subsection{Homology modules}

We are now ready to state and prove the main result of this section. An abbreviated proof was given by Dwyer and Fried in [6], in the special case when $A$ is free Abelian. For the convenience of the reader, we give here a complete proof, modeled on that from [6].

Theorem 9.2. Let $F$ be a finitely generated $\mathbb{C}$-algebra, let $C$ • be a chain complex of finitely generated free modules over $F[H]$, and let $v: H \rightarrow A$ be an epimorphism. Viewing $F[A]$ as a module over $F[H]$ by extension of scalars via $v$, we have

$$
\operatorname{supp} H_{*}\left(C_{\bullet} \otimes_{F[H]} F[A]\right)=\left(v^{*}\right)^{-1}\left(\operatorname{supp} H_{*}\left(C_{\bullet}\right)\right) .
$$

Proof. Set $n=\operatorname{rank} H$ and $r=\operatorname{rank} A$. There are three cases to consider.

Case 1: $H$ is torsion-free. We use induction on $n-r$ to reduce to the case $r=n-1$, in which case $\operatorname{Tors}(A)=\mathbb{Z}_{q}$, for some $q \geq 1$ (if $A$ is torsion-free, $q=1$ ). We then have a short exact sequence of chain complexes,

$$
0 \longrightarrow C_{\bullet} \stackrel{\phi=x^{q}-1}{\longrightarrow} C_{\bullet} \longrightarrow C_{\bullet} \otimes_{F[H]} F[A] \longrightarrow 0,
$$

which yields a long exact sequence of homology groups. Consider the map $\phi_{*}: M \rightarrow$ $M$, where $M=H_{*}\left(C_{\bullet}\right)$, viewed as a module over $F[H]$. Localizing at a maximal ideal $\mathfrak{m}$, we obtain an endomorphism $\phi_{\mathfrak{m}}$ of the finitely-generated module $M_{\mathfrak{m}}$ over the Noetherian ring $F[H]_{\mathfrak{m}}$.

As a standard fact, if $\phi_{\mathfrak{m}}$ is surjective, then $\phi_{\mathfrak{m}}$ is injective. Using the exact sequence

$$
0 \rightarrow \operatorname{ker} \phi_{\mathfrak{m}} \rightarrow M_{\mathfrak{m}} \rightarrow M_{\mathfrak{m}} \rightarrow \operatorname{coker} \phi_{\mathfrak{m}} \rightarrow 0,
$$

we see that coker $\phi_{\mathfrak{m}}=0 \Rightarrow \operatorname{ker} \phi_{\mathfrak{m}}=0$. Therefore, supp ker $\phi_{*} \subseteq \operatorname{supp} \operatorname{coker} \phi_{*}$, and so

$$
\begin{aligned}
\operatorname{supp} H_{*}\left(C \bullet \otimes_{F[H]} F[A]\right) & =\operatorname{supp} \operatorname{coker} \phi_{*} \cup \operatorname{supp} \operatorname{ker} \phi_{*} \\
& =\operatorname{supp} \operatorname{coker} \phi_{*} \\
& =\operatorname{supp} M /\left(x^{q}-1\right) M \\
& =(\operatorname{supp} M) \cap Z\left(x^{q}-1\right) \\
& =(\operatorname{supp} M) \cap \operatorname{im}(\hat{v}) .
\end{aligned}
$$

When $n-r>1$, one can change the basis of $H$ and $\bar{A}$ so that, the epimorphism $v: H \rightarrow A$ is the composite

$$
H^{\prime} \oplus \mathbb{Z} \stackrel{v_{1}}{\longrightarrow} H^{\prime} \oplus \mathbb{Z}_{q} \stackrel{\nu_{2}}{\longrightarrow} A^{\prime} \oplus \mathbb{Z}_{q},
$$

where $v_{1}$ is of the form $\left(\begin{array}{cc}\text { id } & 0 \\ 0 & \left.v\right|_{\mathbb{Z}}\end{array}\right)$, and $\nu_{2}$ is of the form $\left(\begin{array}{cc}\left.\nu\right|_{H^{\prime}} & 0 \\ 0 & \text { id }\end{array}\right)$. By the induction hypothesis, equality (9.4) holds for $v_{1}$ and $v_{2}$. Thus, the theorem holds for the map $v=v_{2} \circ v_{1}$. 
Case 2: $H$ is finite. In this situation, $v: H \rightarrow A$ is an epimorphism between two finite Abelian groups. As above, $v$ induces a ring epimorphism $v: F[H] \rightarrow F[A]$. The corresponding map, $i=v^{*}: \operatorname{maxSpec} F[A] \hookrightarrow \operatorname{maxSpec} F[H]$, is a closed immersion. Consider the commuting diagram

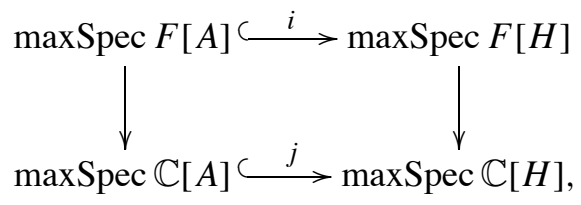

where the morphism $j$ is induced by $v: H \rightarrow A$. Clearly, $j$ is an open immersion. By commutativity of (9.5), we have that $\operatorname{maxSpec} F[A]$ is an open subset of $\max \operatorname{Spec} F[H]$.

It suffices to show that

$$
\operatorname{supp}\left(H_{k}\left(i^{*} \tilde{C}_{\bullet}\right)\right)=i^{-1}\left(\operatorname{supp} H_{k}\left(\tilde{C}_{\bullet}\right)\right)
$$

for any $k \in \mathbb{Z}$, where $\tilde{C}_{\bullet}$ is the sheaf of modules over $\max \operatorname{Spec}(F[H])$ corresponding to the module $C_{\bullet}$ over $F[H]$, and $i^{*} \tilde{C}_{\bullet}$ is the sheaf of modules over $\max \operatorname{Spec}(F[A])$ obtained by pulling back the sheaf $\tilde{C}_{\bullet}$.

For any $\mathfrak{m} \in \max \operatorname{Spec}(F[A])$, we have $\left(H_{k}\left(\tilde{C}_{\bullet}\right)\right)_{\mathfrak{m}}=H_{k}\left(\left(\tilde{C}_{\bullet}\right)_{\mathfrak{m}}\right)$, since localization is an exact functor, and also $\left(\tilde{C}_{\bullet}\right)_{\mathfrak{m}}=\left(i^{*} \tilde{C}_{\bullet}\right)_{\mathfrak{m}}$, since $i$ is an open immersion. Thus,

$$
\begin{aligned}
\operatorname{supp} H_{k}\left(i^{*} \tilde{C}_{\bullet}\right) & =\left\{\mathfrak{m} \in \max \operatorname{Spec}(F[A]) \mid\left(H_{k}\left(i^{*} \tilde{C}_{\bullet}\right)\right)_{\mathfrak{m}} \neq 0\right\} \\
& =\left\{\mathfrak{m} \in \max \operatorname{Spec}(F[A]) \mid\left(i^{*} \tilde{C}_{\bullet}\right)_{\mathfrak{m}} \text { is not exact at } k\right\} \\
& =\left\{\mathfrak{m} \in \max \operatorname{Spec}(F[A]) \mid\left(H_{k}\left(\tilde{C}_{\bullet}\right)\right)_{\mathfrak{m}} \neq 0\right\} \\
& =\operatorname{supp} H_{k}\left(\tilde{C}_{\bullet}\right) \cap \max \operatorname{Spec}(F[A]) \\
& =i^{-1}\left(\operatorname{supp} H_{k}\left(\tilde{C}_{\bullet}\right)\right) .
\end{aligned}
$$

Case 3: $H$ is arbitrary. As in the proof of Theorem 3.2, we can choose splittings $H=\bar{H} \oplus \operatorname{Tors}(H)$ and $A=\bar{A} \oplus \operatorname{Tors}(A)$ such that $\bar{H}=\bar{A} \oplus H^{\prime}$, and the epimorphism $v: H \rightarrow A$ is the composite

$$
\bar{A} \oplus H^{\prime} \oplus \operatorname{Tors}(H) \stackrel{\nu_{1}}{\longrightarrow} \bar{A} \oplus v\left(H^{\prime}\right) \oplus \operatorname{Tors}(H) \stackrel{\nu_{2}}{\longrightarrow} \bar{A} \oplus \operatorname{Tors}(A),
$$

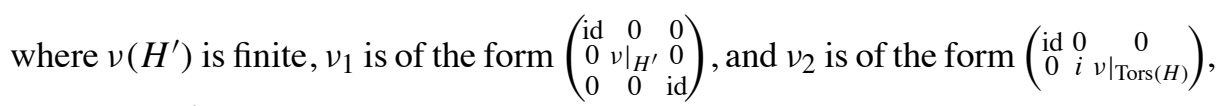
with $i: v\left(H^{\prime}\right) \hookrightarrow \operatorname{Tors}(A)$ the inclusion map.

Set $F=\mathbb{C}[\bar{A} \oplus \operatorname{Tors}(H)]$; by Case 1 , equality (9.4) holds for $v_{1}$. Now set $F=\mathbb{C}[\bar{A}]$; by Case 2, equality (9.4) holds for $\nu_{2}$. Thus, the theorem holds for the map $v=v_{2} \circ v_{1}$. 


\subsection{Finite supports}

We conclude this section with a result which is presumably folklore. For completeness, we include a proof.

Proposition 9.3. Let $A$ be a finitely generated Abelian group, and let $M$ be a finitely generated module over the group ring $S=\mathbb{C}[A]$. Then $M$ as a $\mathbb{C}$-vector space is finite-dimensional if and only if $\operatorname{supp}(M)$ is finite.

Proof. Since $M$ is a finitely generated $S$-module, we can argue by induction on the number of generators of $M$. Using the short exact sequence $0 \rightarrow\langle m\rangle \rightarrow$ $M \rightarrow M /\langle m\rangle \rightarrow 0$, where $m$ is a generator of $M$, and the fact that $\operatorname{supp}(M)=$ $\operatorname{supp}(\langle m\rangle) \cup \operatorname{supp}(M /\langle m\rangle)$, we see that it suffices to consider the case when $M$ is a cyclic module. In this case, $M=S / \operatorname{ann}(M)$ and $\operatorname{supp}(M)=Z(\operatorname{ann}(M))=$ $\operatorname{maxSpec}(S / \operatorname{ann}(M))$. From the assumption that $\operatorname{supp} M$ is finite, and using the Noether Normalization lemma, we infer that $S / \operatorname{ann}(M)$ is an integral extension of $\mathbb{C}$. Thus, $\operatorname{dim}_{\mathbb{C}}(S / \operatorname{ann}(M))<\infty$.

Conversely, suppose $\operatorname{supp}(M)$ is infinite. Then $\max \operatorname{Spec}(S / \operatorname{ann}(M))$ is infinite, which implies $\max \operatorname{Spec}(S / \operatorname{ann}(M))$ has positive dimension. Choose a prime ideal $\mathfrak{p}$ containing ann $(M)$, such that the Krull dimension of $S / \mathfrak{p}$ is positive. From the condition that $\operatorname{dim}_{\mathbb{C}} S / \operatorname{ann}(M)<\infty$, we deduce that $\operatorname{dim}_{\mathbb{C}} S / \mathfrak{p}<\infty$. By the Noether Normalization lemma, $S / \mathfrak{p}$ is an integral extension of $\mathbb{C}\left[x_{1}, \ldots, x_{n}\right]$, with $n>0$. Thus, $\operatorname{dim}_{\mathbb{C}} S / \mathfrak{p}=\infty$. This is a contradiction, so we are done.

\section{Characteristic varieties and generalized Dwyer-Fried sets}

In this section we finally tie together several strands, and show how to determine the sets $\Omega_{A}^{i}(X)$ in terms of the jump loci for homology in rank-1 local systems on $X$.

\subsection{The equivariant chain complex}

Let $X$ be a connected CW-complex. As usual, we will assume that $X$ has finite $k$-skeleton, for some $k \geq 1$. Without loss of generality, we may assume that $X$ has a single 0 -cell $e^{0}$, which we will take as our basepoint $x_{0}$. Moreover, we may assume that all attaching maps $\left(S^{i}, *\right) \rightarrow\left(X^{i}, x_{0}\right)$ are basepoint-preserving. Let $G=\pi_{1}\left(X, x_{0}\right)$ be the fundamental group of $X$, and denote by $\left(C_{i}(X, \mathbb{C}), \partial_{i}\right)_{i \geq 0}$ the cellular chain complex of $X$, with coefficients in $\mathbb{C}$.

Let $p: X^{\mathrm{ab}} \rightarrow X$ be the universal Abelian cover. The cell structure on $X$ lifts in a natural fashion to a cell structure on $X^{\mathrm{ab}}$. Fixing a lift $\tilde{x}_{0} \in p^{-1}\left(x_{0}\right)$ identifies the group $H=G_{\mathrm{ab}}$ with the group of deck transformations of $X^{\mathrm{ab}}$, which permute the cells. Therefore, we may view the cellular chain complex $C_{\bullet}=C_{\bullet}\left(X^{\mathrm{ab}}, \mathbb{C}\right)$ as a chain complex of left-modules over the group algebra $R=\mathbb{C}[H]$. This chain complex has the form

$$
\cdots \rightarrow C_{i} \stackrel{\tilde{\partial}_{i}}{\longrightarrow} C_{i-1} \longrightarrow \cdots \longrightarrow C_{2} \stackrel{\tilde{\partial}_{2}}{\longrightarrow} C_{1} \stackrel{\tilde{\partial}_{1}}{\longrightarrow} C_{0} .
$$


The first two boundary maps can be written down explicitly. Let $e_{1}^{1}, \ldots, e_{m}^{1}$ be the 1-cells of $X$. Since we have a single 0 -cell, each $e_{i}^{1}$ is a loop, representing an element $x_{i} \in G$. Let $\tilde{e}^{0}=\tilde{x}_{0}$, and let $\tilde{e}_{i}^{1}$ be the lift of $e_{i}^{1}$ at $\tilde{x}_{0}$; then $\tilde{\partial}_{1}\left(\tilde{e}_{i}^{1}\right)=$ $\left(x_{i}-1\right) \tilde{e}^{0}$. Next, let $e^{2}$ be a 2-cell, and let $\tilde{e}^{2}$ be its lift at $\tilde{x}_{0}$; then

$$
\tilde{\partial}_{2}\left(\tilde{e}^{2}\right)=\sum_{i=1}^{m} \phi\left(\partial r / \partial x_{i}\right) \cdot \tilde{e}_{i}^{1},
$$

where $r$ is the word in the free group $F_{m}=\left\langle x_{1}, \ldots, x_{m}\right\rangle$ determined by the attaching map of the 2-cell, $\partial r / \partial x_{i} \in \mathbb{C}\left[F_{m}\right]$ are the Fox derivatives of $r$, and $\phi: \mathbb{C}\left[F_{m}\right] \rightarrow \mathbb{C}[H]$ is the extension to group rings of the projection map $F_{m} \rightarrow$ $G \stackrel{\mathrm{ab}}{\rightarrow} H$, see [7].

\subsection{Characteristic varieties}

Since $X$ has finite 1-skeleton, the group $H=H_{1}(X, \mathbb{Z})$ is finitely generated, and its dual, $\widehat{H}=\operatorname{Hom}\left(H, \mathbb{C}^{*}\right)$, is a complex algebraic group. As is well-known, the character group $\widehat{H}$ parametrizes rank-1 local systems on $X$ : given a character $\rho: H \rightarrow \mathbb{C}^{*}$, denote by $\mathbb{C}_{\rho}$ the 1 -dimensional $\mathbb{C}$-vector space, viewed as a right $R$-module via $a \cdot g=\rho(g) a$, for $g \in H$ and $a \in \mathbb{C}$. The homology groups of $X$ with coefficients in $\mathbb{C}_{\rho}$ are then defined as

$$
H_{i}\left(X, \mathbb{C}_{\rho}\right):=H_{i}\left(C_{\bullet}\left(X^{\mathrm{ab}}, \mathbb{C}\right) \otimes_{R} \mathbb{C}_{\rho}\right) .
$$

Definition 10.1. The characteristic varieties of $X$ (over $\mathbb{C}$ ) are the sets

$$
V^{i}(X)=\left\{\rho \in \operatorname{Hom}\left(H, \mathbb{C}^{*}\right) \mid \operatorname{dim}_{\mathbb{C}} H_{j}\left(X, \mathbb{C}_{\rho}\right) \neq 0 \text { for some } 1 \leq j \leq i\right\},
$$

The identity component of the character group $T=\widehat{H}$ is a complex algebraic torus, which we will denote by $T_{0}$. Let $\bar{H}=H / \operatorname{Tors}(H)$ be the maximal torsionfree quotient of $H$. The projection map $\pi: H \rightarrow \bar{H}$ induces an identification $\hat{\pi}: \widehat{\widehat{H}} \stackrel{\simeq}{\lessgtr} \widehat{H}_{0}$. Denote by $W^{i}(X)$ the intersection of $V^{i}(X)$ with $T_{0}=\widehat{H}_{0}$. If $H$ is torsion-free, then $W^{i}(X)=V^{i}(X)$; in general, though, the two varieties differ.

For each $1 \leq i \leq k$, the set $V^{i}(X)$ is a Zariski closed subset of the complex algebraic group $T$, and $W^{i}(X)$ is a Zariski closed subset of the complex algebraic torus $T_{0}$. Up to isomorphism, these varieties depend only on the homotopy type of $X$. Consequently, we may define the characteristic varieties of a group $G$ admitting a classifying space $K(G, 1)$ with finite $k$-skeleton as $V^{i}(G)=V^{i}(K(G, 1))$, for $i \leq k$. It is readily seen that $V^{1}(X)=V^{1}\left(\pi_{1}(X)\right)$. For more details on all this, we refer to [14].

The characteristic varieties of a space can be reinterpreted as the support varieties of its Alexander invariants, as follows:

Theorem 10.2 ( [13]). For each $1 \leq i \leq k$, the characteristic variety $V^{i}(X)$ coincides with the support of the $\mathbb{C}[H]$-module $\bigoplus_{j=1}^{i} H_{j}\left(X^{\mathrm{ab}}, \mathbb{C}\right)$, while $W^{i}(X)$ coincides with the support of the $\mathbb{C}[\bar{H}]$-module $\bigoplus_{j=1}^{i} H_{j}\left(X^{\mathrm{fab}}, \mathbb{C}\right)$. 


\subsection{The first characteristic variety of a group}

Let $G$ be a finitely presented group. The chain complex (10.1) corresponding to a presentation $G=\left\langle x_{1}, \ldots, x_{q} \mid r_{1}, \ldots, r_{m}\right\rangle$ has second boundary map, $\tilde{\partial}_{2}$, an $m$ by $q$ matrix, with rows given by (10.2). Making use of Theorem 10.2, we see that $V^{1}(G)$ is defined by the vanishing of the codimension 1 minors of the Alexander matrix $\tilde{\partial}_{2}$, at least away from the trivial character 1 . This interpretation allows us to construct groups with fairly complicated characteristic varieties:

Lemma 10.3. Let $f=f\left(t_{1}, \ldots, t_{n}\right)$ be a Laurent polynomial with integral coefficients. There is then a finitely presented group $G$ with $G_{\mathrm{ab}}=\mathbb{Z}^{n}$ and $V^{1}(G)=$ $\left\{z \in\left(\mathbb{C}^{*}\right)^{n} \mid f(z)=0\right\} \cup\{1\}$.

Proof. Let $F_{n}=\left\langle x_{1}, \ldots, x_{n}\right\rangle$ be the free group of rank $n$, with Abelianization map $\mathrm{ab}: F_{n} \rightarrow \mathbb{Z}^{n}, x_{k} \mapsto t_{k}$. Recall the following result of R. Lyndon (as recorded in [7]): if $v_{1}, \ldots, v_{n}$ are elements in the ring $\mathbb{Z}\left[\mathbb{Z}^{n}\right]=\mathbb{Z}\left[t_{1}^{ \pm 1}, \ldots, t_{n}^{ \pm 1}\right]$, satisfying the equation $\sum_{k=1}^{n}\left(t_{k}-1\right) v_{k}=0$, then there exists an element $r \in F_{n}^{\prime}$ such that $v_{k}=\mathrm{ab}\left(\partial r / \partial x_{k}\right)$, for $1 \leq k \leq n$.

Making use of this result, we may find elements $r_{i, j} \in F_{n}^{\prime}, 1 \leq i<j \leq n$ such that

$$
\operatorname{ab}\left(\partial r_{i, j} / \partial x_{k}\right)= \begin{cases}f \cdot\left(t_{i}-1\right), & \text { if } k=i \\ f \cdot\left(1-t_{j}\right), & \text { if } k=j \\ 0, & \text { otherwise. }\end{cases}
$$

It is now readily checked that the group $G$ with generators $x_{1}, \ldots, x_{n}$ and relations $r_{i j}$ has the prescribed first characteristic variety.

In certain situations, one may realize a Laurent polynomial as the defining equation for the characteristic variety by a more geometric construction:

Example 10.4. Let $L$ be an $n$-component link in $S^{3}$, with complement $X$. Choosing orientations on the link components yields a meridian basis for $H_{1}(X, \mathbb{Z})=\mathbb{Z}^{n}$. Then

$$
V^{1}(X)=\left\{z \in\left(\mathbb{C}^{*}\right)^{n} \mid \Delta_{L}(z)=0\right\} \cup\{1\},
$$

where $\Delta_{L}=\Delta_{L}\left(t_{1}, \ldots, t_{n}\right)$ is the (multi-variable) Alexander polynomial of the link.

\subsection{A formula for the generalized Dwyer-Fried sets}

Recall that, in Definition 8.5 we associated to each subvariety $W \subset \widehat{H}$, and each Abelian group $A$ a subset

$$
\Upsilon_{A}(W)=\{[v] \in \Gamma(H, A) \mid \operatorname{dim}(\operatorname{im}(\hat{v}) \cap W)>0\} .
$$

The next theorem expresses the Dwyer-Fried sets $\Omega_{A}^{i}(X)$ in terms of the $\Upsilon$-sets associated to the $i$-th characteristic variety of $X$. 
Theorem 10.5. Let $X$ be a connected $C W$-complex, with finite $k$-skeleton. Set $G=$ $\pi_{1}\left(X, x_{0}\right)$ and $H=G_{\mathrm{ab}}$. For any Abelian group $A$, and for any $i \leq k$,

$$
\Omega_{A}^{i}(X)=\Gamma(H, A) \backslash \Upsilon_{A}\left(V^{i}(X)\right) .
$$

Proof. Fix an epimorphism $v: H \rightarrow A$, and let $X^{v} \rightarrow X$ be the corresponding cover. Recall the cellular chain complex $C_{\bullet}=C_{\bullet}\left(X^{\mathrm{ab}}, \mathbb{C}\right)$ is a chain complex of left modules over the ring $R=\mathbb{C}[H]$. If we set $S=\mathbb{C}[A]$, the cellular chain complex $C_{\bullet}\left(X^{\nu}, \mathbb{C}\right)$ can be written as $C_{\bullet} \otimes_{R} S$, where $S$ is viewed as a right $R$ module via extension of scalars by $\nu$.

Consider the $S$-module

$$
M=\bigoplus_{j=1}^{i} H_{j}\left(X^{\nu}, \mathbb{C}\right)=\bigoplus_{j=1}^{i} H_{j}\left(C \bullet \otimes_{R} S\right) .
$$

By definition, [v] belongs to $\Omega_{A}^{i}(X)$ if and only if the Betti numbers $b_{1}\left(X^{v}\right), \ldots$ $\ldots, b_{i}\left(X^{\nu}\right)$ are all finite, i.e., $\operatorname{dim}_{\mathbb{C}} M<\infty$. By Proposition 9.3, this condition is equivalent to $\operatorname{supp} M$ being finite.

Now let $\nu^{*}: \max \operatorname{Spec}(S) \hookrightarrow \max \operatorname{Spec}(R)$ be the induced morphism between the corresponding affine schemes. We then have

$$
\begin{aligned}
\operatorname{supp} M & =\left(v^{*}\right)^{-1} \operatorname{supp}\left(\bigoplus_{j=1}^{i} H_{j}\left(C_{\bullet}\right)\right) & & \text { by Theorem } 9.2 \\
& \cong \operatorname{im}(\hat{v}) \cap Z\left(\operatorname{ann}\left(\bigoplus_{j=1}^{i} H_{j}\left(X^{\mathrm{ab}}, \mathbb{C}\right)\right)\right) & & \text { by Lemma } 9.1 \\
& =\operatorname{im}(\hat{v}) \cap V^{i}(X) & & \text { by Theorem 10.2. }
\end{aligned}
$$

This ends the proof.

Remark 10.6. If $H$ is torsion-free, then $W^{i}(X)=V^{i}(X)$; thus, the set $\Omega_{A}^{i}(X)$ depends only the variety $W^{i}(X)$ and the Abelian group $A$. On the other hand, if $\operatorname{Tors}(H) \neq 0$, the variety $W^{i}(X)$ may be strictly included in $V^{i}(X)$, in which case the set $\Omega_{A}^{i}(X)$ may depend on information not carried by $W^{i}(X)$. We shall see examples of this phenomenon in Subsection 11.3.

\subsection{An upper bound for the $\Omega$-sets}

We now give a computable "upper bound" for the generalized Dwyer-Fried sets $\Omega_{A}^{i}(X)$, in terms of the sets introduced in Subsection 8.1.

Theorem 10.7. Let $X$ be a connected $C W$-complex with finite $k$-skeleton. Set $H=$ $H_{1}(X, \mathbb{Z})$, and fix a degree $i \leq k$. Let $A$ be a quotient of $H$. Then

$$
\Omega_{A}^{i}(X) \subseteq \Gamma(H, A) \backslash U_{A}\left(V^{i}(X)\right) .
$$

Proof. Follows at once from Proposition 8.6 and Theorem 10.5. 
In general, inclusion (10.6) is not an equality. Indeed, the fact that $V(\operatorname{ker} v) \cap$ $V^{i}(X)$ is infinite cannot guarantee there is an algebraic subgroup in the intersection. Here is a concrete instance of this phenomenon:

Example 10.8. Using Lemma 10.3, we may find a 3-generator, 3-relator group $G$ with Abelianization $H=\mathbb{Z}^{3}$ and characteristic variety

$$
V^{1}(G)=\left\{\left(t_{1}, t_{2}, t_{3}\right) \in\left(\mathbb{C}^{*}\right)^{3} \mid\left(t_{2}-1\right)=\left(t_{1}+1\right)\left(t_{3}-1\right)\right\} .
$$

This variety has a single irreducible component, which is a complex torus passing through the origin; nevertheless, this component does not embed as an algebraic subgroup in $\left(\mathbb{C}^{*}\right)^{3}$.

There are 4 maximal, positive-dimensional torsion-translated subtori contained in $V^{1}(G)$, namely $\eta_{1} V\left(\overline{\xi_{1}}\right), \ldots, \eta_{4} V\left(\overline{\xi_{4}}\right)$, where $\xi_{1}, \ldots, \xi_{4}$ are the subgroups of $\mathbb{Z}^{3}$ given by

$$
\xi_{1}=\operatorname{im}\left(\begin{array}{ll}
2 & 0 \\
0 & 1 \\
0 & 0
\end{array}\right), \quad \xi_{2}=\operatorname{im}\left(\begin{array}{ll}
0 & 0 \\
1 & 0 \\
0 & 1
\end{array}\right), \quad \xi_{3}=\operatorname{im}\left(\begin{array}{cc}
2 & -1 \\
-1 & 0 \\
0 & 1
\end{array}\right), \quad \xi_{4}=\operatorname{im}\left(\begin{array}{cc}
2 & -2 \\
-1 & 0 \\
0 & 2
\end{array}\right)
$$

and $\eta_{1}=(-1,1,1), \eta_{2}=\eta_{3}=(1,1,1), \eta_{4}=(-1,1,-1)$.

We claim that, for $A=\mathbb{Z}^{2} \oplus \mathbb{Z}_{2}$, inclusion (10.6) from Theorem 10.7 is strict, i.e.,

$$
\Omega_{A}^{1}(G) \varsubsetneqq \Gamma(H, A) \backslash U_{A}\left(V^{1}(G)\right)
$$

To prove this claim, consider the epimorphism $v: \mathbb{Z}^{3} \rightarrow \mathbb{Z}^{2} \oplus \mathbb{Z}_{2}$ given by the matrix $\left(\begin{array}{lll}1 & 0 & 0 \\ 0 & 0 & 1 \\ 0 & 1 & 0\end{array}\right)$. Note that $\operatorname{ker}(v)=\operatorname{im}\left(\begin{array}{l}0 \\ 2 \\ 0\end{array}\right)$, and so $\operatorname{im}(\hat{v})=\left\{t \in\left(\mathbb{C}^{*}\right)^{3} \mid t_{2}= \pm 1\right\}$. The intersection of $\operatorname{im}(\hat{v})$ with $V^{1}(G)$ consists of all points of the form $\left(t_{1}, \pm 1, t_{3}\right)$ with $\left(t_{1}+1\right)\left(t_{3}-1\right)$ equal to 0 or -2 . Clearly, this is an infinite set; therefore, $[\nu] \notin \Omega_{A}^{1}(G)$.

On the other hand, $\operatorname{im}(\hat{v}) \cap \eta_{1} V\left(\overline{\xi_{1}}\right)=\operatorname{im}(\hat{v}) \cap \eta_{2} V\left(\overline{\xi_{2}}\right)=\emptyset$, and $\operatorname{rank}(\operatorname{ker}(\nu)+$ $\left.\xi_{3}\right)=\operatorname{rank}\left(\operatorname{ker}(\nu)+\xi_{4}\right)=\operatorname{rank}(H)$. Hence, $\operatorname{im}(\hat{v}) \cap \eta_{j} V\left(\overline{\xi_{j}}\right)$ is finite, for all $j$. In view of Lemma 8.2, we conclude that $[\nu] \notin U_{A}\left(V^{1}(G)\right)$.

\section{Comparison with the classical Dwyer-Fried invariants}

\subsection{The Dwyer-Fried invariants $\Omega_{r}^{i}(X)$}

In the case of free Abelian covers and the usual $\Omega$-sets, Theorem 10.5 allows us to recover the following result from $[6,13,14]$.

Corollary 11.1. Set $n=b_{1}(X)$. Then, for all $r \geq 1$,

$$
\Omega_{r}^{i}(X)=\left\{[\nu] \in \operatorname{Gr}_{r}\left(\mathbb{Z}^{n}\right) \mid \operatorname{im}(\hat{v}) \cap W^{i}(X) \text { is finite }\right\} .
$$

Using now the identification from Example 7.3, and taking into account Lemma 6.5 , Theorem 10.7 yields the following corollary. 
Corollary $11.2([13,14])$. Let $X$ be a connected $C W$-complex with finite $k$-skeleton, and set $n=b_{1}(X)$. Then $\Omega_{r}^{i}(X) \subseteq \mathbb{Q P}^{n-1} \backslash \mathbb{P}\left(\tau_{1}\left(W^{i}(X)\right)\right)$, for all $i \leq k$ and all $r \leq n$.

Remark 11.3. As noted in [14], if the variety $W^{i}(X)$ is a union of algebraic subtori, then the Dwyer-Fried sets $\Omega_{r}^{i}(X)$ are open subsets of $\mathrm{Gr}_{r}\left(\mathbb{Z}^{n}\right)$, for all $r \geq 1$. In general, though, examples from $[6,14]$ show that the sets $\Omega_{r}^{i}(X)$ with $r>1$ need not be open. On the other hand, as noted in $[6,13,14]$, the sets $\Omega_{1}^{i}(X)$ are always open subsets of $\mathrm{Gr}_{1}\left(\mathbb{Z}^{n}\right)=\mathbb{Q} P^{n-1}$. We will come back to this phenomenon in Section 12 , in a more general context.

\subsection{The comparison diagram, revisited}

As may be expected, the generalized Dwyer-Fried invariants carry more information about the homotopy type of a space and the homological finiteness properties of its regular Abelian covers than the classical ones. To make this more precise, let $A$ be a quotient of the group $H=H_{1}(X, \mathbb{Z})$. Fix a decomposition $A=\bar{A} \oplus \operatorname{Tors}(A)$, and identify $\Omega_{r}^{i}(X)=\Omega_{\bar{A}}^{i}(X)$, where $r=\operatorname{rank}(A)$. As we saw in Section 4.4, for each $i \leq k$ we have a comparison diagram

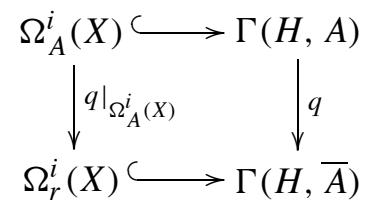

between the respective Dwyer-Fried invariants, viewed as subsets of the parameter sets for regular $A$-covers and $\bar{A}$-covers, respectively.

We are interested in describing conditions under which the set $\Omega_{A}^{i}(X)$ contains more information than $\Omega_{r}^{i}(X)$. This typically happens when the comparison diagram (11.1) is not a pull-back diagram, i.e., there is a point $[\bar{v}] \in \Omega_{r}^{i}(X)$ for which the fiber $q^{-1}([\bar{v}])$ is not included in $\Omega_{A}^{i}(X)$. In fact, the number of points in the fiber which lie in $\Omega_{A}^{i}(X)$ may vary as we move about $\Omega_{r}^{i}(X)$.

In view of Theorem 10.5, we have the following criterion:

Proposition 11.4. Diagram (11.1) fails to be a pull-back diagram if and only if $q\left(\Upsilon_{A}\left(V^{i}(X)\right)\right.$ is not included in $\Upsilon_{r}\left(V^{i}(X)\right.$, i.e., there is an epimorphism $v: H \rightarrow$ A such that

$$
\operatorname{dim}\left(\operatorname{im} \hat{v} \cap V^{i}(X)\right)>0, \quad \text { yet } \quad \operatorname{dim}\left(\operatorname{im} \hat{\bar{v}} \cap V^{i}(X)\right)=0 .
$$

From Proposition 4.10, we know diagram (11.1) is a pull-back diagram precisely when the homological finiteness of an arbitrary $A$-cover of $X$ can be tested through the corresponding $\bar{A}$-cover. In order to quantify the discrepancy between these two types of homological finiteness properties, let us define the "singular set"

$$
\Sigma_{A}^{i}(X)=\left\{[\bar{v}] \in \Omega_{\bar{A}}^{i}(X) \mid \#\left(q^{-1}([\bar{v}]) \cap \Omega_{A}^{i}(X)\right)<\#\left(q^{-1}([\bar{v}])\right)\right\} .
$$


We then have:

$$
\Sigma_{A}^{i}(X)=q\left(\Omega_{A}^{i}(X)^{c}\right) \cap \Omega_{\bar{A}}^{i}(X) .
$$

\subsection{Maximal Abelian versus free Abelian covers}

We now investigate the relationship between the finiteness of the Betti numbers of the maximal Abelian cover $X^{\mathrm{ab}}$ and the finiteness of the Betti numbers of the corresponding free Abelian cover $X^{\text {fab }}$ of our space $X$.

As before, write $H=H_{1}(X, \mathbb{Z})$ and identify the character group $\widehat{H}$ with $\left(\mathbb{C}^{*}\right)^{n} \times \operatorname{Tors}(H)$, where $n=b_{1}(X)$.

Proposition 11.5. Suppose Tors $(H) \neq 0$. Furthermore, assume that $W^{i}(X)$ is finite, whereas $V^{i}(X)=W^{i}(X) \cup\left(\widehat{H} \backslash \widehat{H}_{0}\right)$. Then

(i) $\Omega_{r}^{i}(X)=\mathrm{Gr}_{r}\left(\mathbb{Z}^{n}\right)$, for all $r \geq 1$,

(ii) If $\operatorname{rank}(A)=\operatorname{rank}(H)$ and $\operatorname{Tors}(A) \neq 0$, then $\Omega_{A}^{i}(X)=\emptyset$.

Proof. By Theorem 10.5, an element $[\nu] \in \mathrm{Gr}_{r}\left(\mathbb{Z}^{n}\right)$ belongs to $\Omega_{r}^{i}(X)$ if and only if $\operatorname{im}(\hat{v}) \cap W^{i}(X)$ is finite. By assumption, $W^{i}(X)$ is finite; thus, the intersection $\operatorname{im}(\hat{v}) \cap W^{i}(X)$ is also finite. This proves (i).

Again by Theorem 10.5, an element $[v] \in \Gamma(H, A)$ belongs to $\Omega_{A}^{i}(X)$ if and only if $\operatorname{im}(\hat{v}) \cap V^{i}(X)$ is finite. By assumption, $V^{i}(X)=W^{i}(X) \cup\left(\widehat{H} \backslash \widehat{H}_{0}\right)$; moreover, $\operatorname{rank}(A)=\operatorname{rank}(H)$ and $\operatorname{Tors}(A) \neq 0$. Thus, the intersection $\operatorname{im}(\hat{v}) \cap$ $V^{i}(X)$ contains at least one component of $\widehat{H} \backslash \widehat{H}_{0}$, which is infinite. This proves (ii).

A similar argument yields the following result:

Proposition 11.6. Suppose $H_{1}(X, \mathbb{Z})$ has non-trivial torsion, $W^{1}(X)$ is finite, and $V^{1}(X)$ is infinite. Then $b_{1}\left(X^{\mathrm{fab}}\right)<\infty$, yet $b_{1}\left(X^{\mathrm{ab}}\right)=\infty$.

Proposition 11.7. Suppose $X=X_{1} \vee X_{2}$, where $H_{1}\left(X_{1}, \mathbb{Z}\right)$ is free Abelian and non-trivial, and $H_{1}\left(X_{2}, \mathbb{Z}\right)$ is finite and non-trivial. If $V^{1}\left(X_{1}\right)$ is finite, then $b_{1}\left(X^{\mathrm{fab}}\right)<\infty$, yet $b_{1}\left(X^{\mathrm{ab}}\right)=\infty$.

Proof. Let $H=H_{1}(X, \mathbb{Z})$; then $\bar{H}=H_{1}\left(X_{1}, \mathbb{Z}\right) \neq 0$ and $\operatorname{Tors}(H)=H_{1}\left(X_{2}, \mathbb{Z}\right) \neq$ 0 . Thus, the character group $\widehat{H}$ decomposes as $\widehat{H}_{0} \times \operatorname{Tors}(H)$, with both factors non-trivial.

Now, $W^{1}(X)=V^{1}\left(X_{1}\right) \times\{1\}$ is finite, and thus $\Omega \frac{1}{H}(X)$ is a singleton. On the other hand, $V^{1}(X)=W^{1}(X) \cup \widehat{H}_{0} \times(\operatorname{Tors}(H) \backslash\{1\})$ is infinite, and thus $\Omega_{H}^{1}(X)=\emptyset$.

Example 11.8. Consider the CW-complexes $X=S^{1} \vee \mathbb{R} \mathbb{P}^{2}$ and $Y=S^{1} \times \mathbb{R P}^{2}$. Then $H_{1}(X, \mathbb{Z}) \cong H_{1}(Y, \mathbb{Z}) \cong \mathbb{Z} \times \mathbb{Z}_{2}$. Clearly, the free Abelian covers $X^{\text {fab }}$ and $Y^{\text {fab }}$ are rationally acyclic; thus both $\Omega_{1}^{i}(X)$ and $\Omega_{1}^{i}(Y)$ consist of a single point, for all $i \geq 0$. On the other hand, the free Abelian cover $X^{\mathrm{ab}}$ has the homotopy type of a 
countable wedge of $S^{1}$ 's and $\mathbb{R P}^{2}$, s, whereas $Y^{\mathrm{ab}} \simeq S^{2}$. Therefore, $\Omega_{\mathbb{Z} \oplus \mathbb{Z}_{2}}(X)=\emptyset$, while $\Omega_{\mathbb{Z} \oplus \mathbb{Z}_{2}}^{1}(Y)=$ \{point .

This example shows that the generalized Dwyer-Fried invariants $\Omega_{A}^{i}(X)$ may contain more information than the classical ones.

\section{The rank-1 case}

In this section, we discuss in more detail the invariants $\Omega_{A}^{i}(X)$ in the case when $A$ has rank-1, and push the analysis even further in some particularly simple situations.

\subsection{A simplified formula for $\Omega_{A}^{i}(X)$}

Recall that, for a finitely generated Abelian group $A$, the integer $c(A)$ denotes the largest order of an element in $A$. Recall also that, for every subvariety $W \subset \widehat{H}$ and each index $d \geq 1$, we have a subset $U_{A, d}(W) \subset \Gamma(H, A)$, described geometrically in Lemma 8.2.

Theorem 12.1. Let $X$ be a connected $C W$-complex with finite $k$-skeleton. Set $H=$ $H_{1}(X, \mathbb{Z})$, and fix a degree $i \leq k$. If $\operatorname{rank}(A)=1$, then

$$
\Omega_{A}^{i}(X)=\Gamma(H, A) \backslash U_{A, c(A)}\left(V^{i}(X)\right) .
$$

Proof. The inclusion $\subseteq$ follows from Theorem 10.7, so we only need to prove the opposite inclusion.

Let $v: H \rightarrow A$ be an epimorphism such that $[v] \notin \Omega_{A}^{i}(X)$. By Theorem 10.5, the variety $\operatorname{im} \hat{v} \cap V^{i}(X)$ has positive dimension. Since $\operatorname{rank}(A)=1$, there exists a component of the 1-dimensional algebraic subgroup im $\hat{v}$ contained in $V^{i}(X)$; that is, there exists a character $\rho \in \widehat{\operatorname{Tors}(A)}$ such that

$$
\hat{v}(\rho) \cdot \operatorname{im} \hat{\bar{v}} \subseteq V^{i}(X) .
$$

Now, we may find a primitive subgroup $\chi \leq H$ and a torsion character $\eta \in \widehat{H}$ such that $\eta V(\chi)=\hat{v}(\rho) V(\chi)$ is a maximal translated subtorus in $V^{i}(X)$ which contains $\hat{v}(\rho) \cdot \operatorname{im} \hat{\bar{v}}$. Set

$$
\xi=\epsilon\left(\bigcup_{m \geq 1} \eta^{m} V(\chi)\right) .
$$

Clearly, the subgroup $\xi \leq H$ belongs to $\Xi_{d}\left(V^{i}(X)\right)$, where $d:=\operatorname{ord}(\rho)$ divides $c(A)$. Since $\hat{v}(\rho) V(\chi) \supseteq \hat{v}(\rho) \cdot V(\operatorname{ker}(\bar{v}))$, we must also have $V(\chi) \supseteq V(\operatorname{ker}(\bar{v}))$, and so $[\nu] \in U_{A}(\xi)$. Therefore, $[\nu] \in U_{A, c(A)}\left(V^{i}(X)\right)$, and we are done.

Corollary $12.2([13,14])$. Let $X$ be a connected $C W$-complex with finite $k$-skeleton, and set $n=b_{1}(X)$. Then $\Omega_{1}^{i}(X)=\mathbb{Q P}^{n-1} \backslash \mathbb{P}\left(\tau_{1}\left(W^{i}(X)\right)\right)$, for all $i \leq k$.

In particular, $\Omega_{1}^{i}(X)$ is an open subset of the projective space $\mathbb{Q} P^{n-1}$. 


\subsection{The singular set}

Recall from Section 11.2 that we measure the discrepancy between the generalized Dwyer-Fried invariant $\Omega_{A}^{i}(X)$ and its classical counterpart, $\Omega_{\frac{i}{A}}(X)$, by means of the "singular set," $\Sigma_{A}^{i}(X)=q\left(\Omega_{A}^{i}(X)^{c}\right) \cap \Omega_{\bar{A}}^{i}(X)$. When the group $A$ has rank-1, this set can be expressed more concretely, as follows.

Proposition 12.3. Suppose $H$ is torsion-free, and $\operatorname{rank}(A)=1$. Then $\Sigma_{A}^{i}(X)$ consists of all $[\sigma] \in \Gamma(H, \bar{A})$ satisfying the following two conditions:

(i) $\operatorname{ker}(\sigma) \supseteq \bar{\xi}$, for some $\xi \in \Xi_{c(A)}\left(V^{i}(X)\right)$, and

(ii) $V(\operatorname{ker}(\sigma)) \nsubseteq V^{i}(X)$.

Proof. Without loss of generality, we may assume that $\Gamma(H, A) \neq \emptyset$. From Theorem 12.1, we know that $\Omega_{A}^{i}(X)=\Gamma(H, A) \backslash U$, where $U=U_{A, c(A)}\left(V^{i}(X)\right)$. It follows that $\Sigma_{A}^{i}(X)=q(U) \cap \Omega_{1}^{i}(X)$.

Now let $S$ be the set of all $[\sigma] \in \Gamma(H, \bar{A})$ satisfying conditions (i) and (ii). It suffices to show that $q(U) \cap \Omega_{1}^{i}(X)=S$.

The inclusion $S \supseteq q(U) \cap \Omega_{1}^{i}(X)$ is straightforward. To establish the reverse inclusion, let $\sigma: H \rightarrow \mathbb{Z}$ represent an element in $S$. Condition (ii) implies that $[\sigma] \in \Omega_{1}^{i}(X)$. Recall that $\pi: A \rightarrow \bar{A}$ is the natural projection. To prove that $[\sigma] \in q(U)$, it is enough to find an epimorphism $v: H \rightarrow A$ such that $\pi \circ v=\sigma$ and $V(\operatorname{ker}(v)) \cap \eta V(\bar{\xi}) \neq \emptyset$, where $\eta$ is a generator of $\widehat{\xi / \xi}$.

Set $d:=\operatorname{ord}(\eta)$. Writing $A=\mathbb{Z}_{d_{1}} \oplus \cdots \oplus \mathbb{Z}_{d_{k}}$, with $d_{1}\left|d_{2}\right| \cdots \mid d_{k}$, we have that $d \mid d_{k}$. Denote by $\iota$ the embedding of the cyclic group $\langle\eta\rangle$ into $\widehat{H}$. Under the correspondence from (5.2), there is a map $\check{\iota}: H \rightarrow \mathbb{Z}_{d}$; clearly, this map factors as the composite

$$
H \stackrel{f_{1}}{\longrightarrow} \mathbb{Z}^{n-1} \oplus \mathbb{Z} \stackrel{\operatorname{id} \oplus \kappa_{1}}{\longrightarrow} \mathbb{Z}^{n-1} \oplus \mathbb{Z}_{d_{k}} \stackrel{\left(0 \kappa_{2}\right)}{\longrightarrow} \mathbb{Z}_{d},
$$

for some isomorphism $f_{1}: H \rightarrow \mathbb{Z}^{n}$, where $\kappa_{1}$ and $\kappa_{2}$ are the canonical projections.

Recall we are assuming $\Gamma(H, A) \neq \emptyset$; thus, there is an epimorphism $\gamma: H \rightarrow$ $A$. We then have a commuting diagram

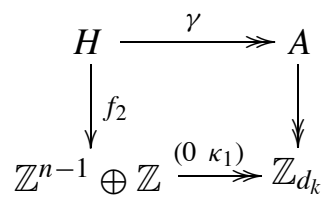

for some isomorphism $f_{2}: H \rightarrow \mathbb{Z}^{n}$. The composite $v=\gamma \circ f_{2}^{-1} \circ f_{1}: H \rightarrow A$, then, is the required epimorphism.

As we shall see in Examples 12.5 and 12.6, the singular set $\Sigma_{A}^{i}(X)$ may be non-empty; in fact, as we shall see in Example 12.7, this set may even be infinite. 


\subsection{A particular case}

Perhaps the simplest situation when such a phenomenon may occur is the one when $H=\mathbb{Z}^{2}$ and $A=\mathbb{Z} \oplus \mathbb{Z}_{2}$. In this case, the set

$$
\Gamma(H / \bar{A}, A / \bar{A})=\operatorname{Epi}\left(\mathbb{Z}, \mathbb{Z}_{2}\right) / \operatorname{Aut}\left(\mathbb{Z}_{2}\right)
$$

is a singleton, and so the map $q_{H}: \Gamma(H, A) \rightarrow \Gamma(H, \bar{A})$ is a bijection. In other words, if $H_{1}(X, \mathbb{Z})=\mathbb{Z}^{2}$, there is a one-to-one correspondence between regular $\mathbb{Z} \oplus \mathbb{Z}_{2}$-covers of $X$ and regular $\mathbb{Z}$-covers of $X$, both parametrized by the projective line $\mathbb{Q} P^{1}=\mathrm{Gr}_{1}\left(\mathbb{Z}^{2}\right)$. The comparison diagram, then, takes the form

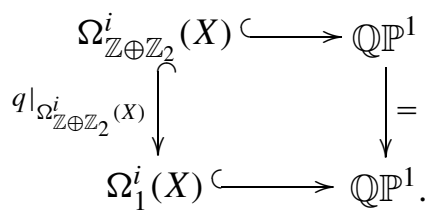

Let $V^{i}(X) \subset\left(\mathbb{C}^{*}\right)^{2}$ be the $i$-th characteristic variety of $X$. For each pair $(a, b) \in$ $\mathbb{Z}^{2}$, consider the (translated) subtori $T_{a, b}^{ \pm}=\left\{\left(t_{1}, t_{2}\right) \in\left(\mathbb{C}^{*}\right)^{2} \mid t_{1}^{a} t_{2}^{b}= \pm 1\right\}$.

Proposition 12.4. Suppose $H=\mathbb{Z}^{2}$ and $A=\mathbb{Z} \oplus \mathbb{Z}_{2}$. Then

$$
\begin{aligned}
& \Omega_{A}^{i}(X)=\left\{(a, b) \in \mathbb{Q P}^{1} \mid T_{-b, a}^{+} \subset V^{i}(X) \text { or } T_{-b, a}^{-} \subset V^{i}(X)\right\}^{c}, \\
& \Omega_{1}^{i}(X)=\left\{(a, b) \in \mathbb{Q P}^{1} \mid T_{-b, a}^{+} \subset V^{i}(X)\right\}^{c} .
\end{aligned}
$$

Proof. Let $(a, b) \in H$ and let $\xi \leq H$ be the subgroup generated by $(-b, a)$. Then $\xi \in \Xi_{1}\left(V^{i}(X)\right)$ if and only if $\xi=\bar{\xi}$ and $V^{i}(X)$ contains a component of the form $t_{1}^{-b} t_{2}^{a}=1$, whereas $\xi \in \Xi_{2}\left(V^{i}(X)\right)$ if and only if $\xi$ has index at most 2 in $\bar{\xi}$ and $V^{i}(X)$ contains a component of the form $t_{1}^{-b} t_{2}^{a}= \pm 1$. The conclusions follow from Theorem 12.1.

In particular, $\Sigma_{\mathbb{Z} \oplus \mathbb{Z}_{2}}^{i}(X)=\Omega_{1}^{i}(X) \backslash \Omega_{\mathbb{Z} \oplus \mathbb{Z}_{2}}^{i}(X)$, and diagram (12.2) is not a pull-back diagram if and only if $V^{i}(X)$ has a component of the form $t_{1}^{a} t_{2}^{b}+1=0$.

A nice class of examples is provided by 2-components links. Let $L=\left(L_{1}, L_{2}\right)$ be such a link, with complement $X_{L}$. As we saw in Example 10.4, the characteristic variety $V^{1}\left(X_{L}\right)$ consists of the zero-locus in $\left(\mathbb{C}^{*}\right)^{2}$ of the Alexander polynomial $\Delta_{L}\left(t_{1}, t_{2}\right)$, together with the identity.

Example 12.5. Let $L$ be the 2-component link denoted $4_{1}^{2}$ in Rolfsen's tables, and let $X_{L}$ be its complement. Then $\Delta_{L}=t_{1}+t_{2}$, and so $V^{1}\left(X_{L}\right)=\{1\} \cup\left\{\left(t_{1}, t_{2}\right) \in\right.$ $\left.\left(\mathbb{C}^{*}\right)^{2} \mid t_{1} t_{2}^{-1}=-1\right\}$. Hence, $\Omega_{1}^{1}\left(X_{L}\right)=\mathbb{Q P}^{1}$, but $\Omega_{\mathbb{Z} \oplus \mathbb{Z}_{2}}^{1}\left(X_{L}\right)=\mathbb{Q P P}^{1} \backslash\{(1,1)\}$. 


\subsection{Another particular case}

The next simplest situation is the one when $H=\mathbb{Z}^{3}$ and $A=\mathbb{Z} \oplus \mathbb{Z}_{2}$. In this case, the set

$$
\Gamma(H / \bar{A}, A / \bar{A})=\operatorname{Epi}\left(\mathbb{Z}^{2}, \mathbb{Z}_{2}\right) / \operatorname{Aut}\left(\mathbb{Z}_{2}\right)=\left(\mathbb{Z}_{2} \oplus \mathbb{Z}_{2}\right)^{*}
$$

consists of 3 elements. In other words, if $H_{1}(X, \mathbb{Z})=\mathbb{Z}^{3}$, there is a three-to-one correspondence between the regular $\mathbb{Z} \oplus \mathbb{Z}_{2}$-covers of $X$ and the regular $\mathbb{Z}$-covers of $X$.

Example 12.6. Let $G$ be the group from Example 10.8. With notation as before, we have that $\Xi_{1}\left(V^{1}(G)\right)=\left\{\xi_{2}, \xi_{3}\right\}$ and $\Xi_{2}\left(V^{1}(G)\right)=\left\{\xi_{1}, \xi_{2}, \xi_{3}, \xi_{4}\right\}$. Consequently, $\mathbb{P}\left(\tau_{1}\left(V^{1}(G)\right)\right)=\{(1,0,0),(1,2,1)\}$, and so $\Omega_{1}^{1}(G)=\mathbb{Q P}^{1} \backslash\{(1,0,0),(1,2,1)\}$.

Let $A=\mathbb{Z} \oplus \mathbb{Z}_{2}$. Given an element $[v] \in \Gamma(H, A)$ such that $\overline{\operatorname{ker}(v)} \supseteq \overline{\xi_{1}}$, we have that $[\bar{v}]=(0,0,1) \in \Omega_{1}^{1}(G)$. Furthermore, $q^{-1}([\bar{v}])$ consists of 3 representative classes: $v_{1}=\left(\begin{array}{lll}0 & 0 & 1 \\ 1 & 0 & 0\end{array}\right), v_{2}=\left(\begin{array}{lll}0 & 0 & 1 \\ 1 & 1 & 0\end{array}\right)$, and $v_{3}=\left(\begin{array}{lll}0 & 0 & 1 \\ 0 & 1 & 0\end{array}\right)$. By calculation $v_{1} \in U$, but $v_{2}, v_{3} \notin U$. Thus,

$$
\Omega_{A}^{1}(G)=\Gamma(H, A) \backslash\left\{q^{-1}(1,0,0), q^{-1}(1,2,1), v_{1}\right\} .
$$

Example 12.7. Consider the group from [14, Example 8.7], with presentation

$$
G=\left\langle x_{1}, x_{2}, x_{3} \mid\left[x_{1}^{2}, x_{2}\right],\left[x_{1}, x_{3}\right], x_{1}\left[x_{2}, x_{3}\right] x_{1}^{-1}\left[x_{2}, x_{3}\right]\right\rangle .
$$

The characteristic variety $V^{1}(G) \subset\left(\mathbb{C}^{*}\right)^{3}$ consists of the origin, together with the translated torus $\left\{\left(t_{1}, t_{2}, t_{3}\right) \in\left(\mathbb{C}^{*}\right)^{3} \mid t_{1}=-1\right\}$; hence, $\Omega_{1}^{1}(G)=\mathbb{Q P}^{2}$.

Let $A=\mathbb{Z} \oplus \mathbb{Z}_{2}$. The singular set $\Sigma=\Sigma_{A}^{1}(G)$ consists of those points $[(0, b, c)] \in \mathbb{Q P}^{2}$ with $b$ and $c$ coprime; thus, $\Sigma \cong \mathbb{Q} \mathbb{P}^{1}$ is infinite. Moreover, the restriction $q: \Omega_{A}^{1}(G) \backslash q^{-1}(\Sigma) \rightarrow \Omega_{1}^{1}(G) \backslash \Sigma$ is three-to-one. On the other hand, if $[\nu] \in q^{-1}(\Sigma)$, then either $v$ is of the form $\left(\begin{array}{ccc}0 & b & c \\ 1 & \epsilon_{2} & \epsilon_{3}\end{array}\right)$, in which case $[\nu] \notin \Omega_{A}^{1}(G)$, or $v$ is of the form $\left(\begin{array}{ccc}0 & b & c \\ 0 & \epsilon_{2} & \epsilon_{3}\end{array}\right)$, in which case $[v] \in \Omega_{A}^{1}(G)$. Thus, the restriction $q: \Omega_{A}^{1}(G) \cap q^{-1}(\Sigma) \rightarrow \Sigma$ is one-to-one.

\section{Translated tori in the characteristic varieties}

Throughout this section, we assume all irreducible components of the characteristic varieties under consideration are (possibly translated) algebraic subgroups of the character group, a condition satisfied by large families of spaces.

\subsection{A refined formula for the $\Omega$-sets}

In Theorem 10.5 we gave a general description of the Dwyer-Freed invariants $\Omega_{A}^{i}(X)$ in terms of the characteristic variety $W=V^{i}(X)$, while in Theorem 10.7 we gave a upper bound for those invariants, in terms of certain sets $\Xi_{d}(W)$ and $U_{A}(\xi)$, introduced in Definitions 6.1 and 7.1, respectively. 
We now refine those results in the special case when all components of the characteristic variety are torsion-translated subgroups of the character group. The next theorem shows that inclusion (10.6) from Theorem 10.7 holds as equality in this case, with the union of all sets $U_{A, d}(W)$ with $d \geq 1$ replaced by a single constituent $U_{A, c}(W)$, for some integer $c$ depending only on $W$ (and not on $A$ ).

Theorem 13.1. Let $X$ be a connected $C W$-complex with finite $k$-skeleton. Set $H=$ $H_{1}(X, \mathbb{Z})$, and fix a degree $i \leq k$. Suppose $V^{i}(X)$ is a union of torsion-translated subgroups of $\widehat{H}$. There is then an integer $c>0$ such that, for every Abelian group A,

$$
\Omega_{A}^{i}(X)=\Gamma(H, A) \backslash U_{A, c}\left(V^{i}(X)\right) .
$$

Proof. By assumption, $V^{i}(X)=\bigcup_{j=1}^{s} \eta_{j} V\left(\xi_{j}\right)$, for some subgroups $\xi_{j} \leq H$ and torsion elements $\eta_{j} \in \widehat{H}$. In the special case when $s=1$, the required equality is proved in Theorem 8.7, with $c=\operatorname{ord}\left(\eta_{1}\right) \cdot c\left(\bar{\xi}_{1} / \xi_{1}\right)$.

The general case follows from a similar argument, with $c$ replaced by the lowest common multiple of $\operatorname{ord}\left(\eta_{1}\right) \cdot c\left(\bar{\xi}_{1} / \xi_{1}\right), \ldots, \operatorname{ord}\left(\eta_{s}\right) \cdot c\left(\bar{\xi}_{s} / \xi_{s}\right)$.

\subsection{Another formula for the $\Omega$-sets}

We now present an alternate formula for computing the sets $\Omega_{A}^{i}(X)$ in the case when the $i$-th characteristic variety of $X$ is a union of torsion-translated subgroups of the character group. Although somewhat similar in spirit to Theorem 13.1, the next theorem uses different ingredients to express the answer.

Theorem 13.2. Let $X$ be a connected $C W$-complex with finite $k$-skeleton. Suppose there is a degree $i \leq k$ such that $V^{i}(X)=\bigcup_{j=1}^{s} \eta_{j} V\left(\xi_{j}\right)$, where $\xi_{1}, \ldots, \xi_{s}$ are subgroups of $H=H_{1}(X, \mathbb{Z})$, and $\eta_{1}, \ldots, \eta_{s}$ are torsion elements in $\widehat{H}$. Then, for each Abelian group $A$,

$$
\Omega_{A}^{i}(X)=\bigcap_{j=1}^{s}\left(\sigma_{A}\left(\xi_{j}\right)^{c} \cup\left\{[v] \in \Gamma(H, A) \mid \epsilon\left(\left\langle\eta_{j}\right\rangle\right) \nsupseteq \operatorname{ker}(v) \cap \xi_{j}\right\}\right) .
$$

Proof. The result follows from Theorems 10.5 and 8.8, as well as formula (8.4).

The simplest situation in which the above theorem applies is that in which there are no translation factors in the subgroups comprising the characteristic variety.

Corollary 13.3. Suppose $V^{i}(X)=V\left(\xi_{1}\right) \cup \cdots \cup V\left(\xi_{s}\right)$ is a union of algebraic subgroups of $\widehat{H}$. Then

$$
\Omega_{A}^{i}(X)=\Gamma(H, A) \backslash \bigcup_{j=1}^{s} q^{-1}\left(\sigma_{\bar{A}}\left(\xi_{j}\right)\right) .
$$

In particular if $X^{v}$ is a free Abelian cover with finite Betti numbers up to degree $i$, then any finite regular Abelian cover of $X^{v}$ has the same finiteness property. 
Proof. By Theorem 13.2,

$$
\Omega_{A}^{i}(X)=\Gamma(H, A) \backslash \bigcup_{j=1}^{s} \sigma_{A}\left(\xi_{j}\right) .
$$

Indeed, for each $j$ we have $\eta_{j}=1$, and thus $\left\{[v] \mid \epsilon\left(\left\langle\eta_{j}\right\rangle\right) \nsupseteq \operatorname{ker}(v) \cap \xi_{j}\right\}$ is the empty set. Applying now Proposition 7.4 ends the proof.

\subsection{Toric complexes}

We illustrate the above corollary with a class of spaces arising in toric topology. Let $L$ be a simplicial complex with $n$ vertices, and let $T^{n}=S^{1} \times \cdots \times S^{1}$ be the $n$-torus, with the standard product cell decomposition. The toric complex associated to $L$, denoted $T_{L}$, is the union of all subcomplexes of the form

$$
T^{\sigma}=\left\{\left(x_{1}, \ldots, x_{n}\right) \in T^{n} \mid x_{i}=* \text { if } i \notin \sigma\right\},
$$

where $\sigma$ runs through the simplices of $L$, and $*$ is the (unique) 0 -cell of $S^{1}$. Clearly, $T_{L}$ is a connected $\mathrm{CW}$-complex, with unique 0 -cell corresponding to the empty simplex $\emptyset$. The fundamental group of $T_{L}$ is the right-angled Artin group

$$
\left.G_{L}=\langle v \in \mathrm{V}| v w=w v \text { if }\{v, w\} \in E\right\rangle,
$$

where $\mathrm{V}$ and $\mathrm{E}$ denote the 0 -cells and 1-cells of $L$. Furthermore, a classifying space for $\pi_{1}\left(T_{L}\right)$ is the toric complex $T_{\Delta(L)}$, where $\Delta(L)$ is the flag complex associated to $L$.

Evidently, $H_{1}\left(T_{L}, \mathbb{Z}\right)=\mathbb{Z}^{n}$; thus, we may identify the character group of $\pi_{1}\left(T_{L}\right)$ with the algebraic torus $\left(\mathbb{C}^{*}\right)^{\mathrm{V}}:=\left(\mathbb{C}^{*}\right)^{n}$. For any subset $\mathrm{W} \subseteq \mathrm{V}$, let $\left(\mathbb{C}^{*}\right)^{\mathrm{W}} \subseteq\left(\mathbb{C}^{*}\right)^{\mathrm{V}}$ be the corresponding subtorus; in particular, $\left(\mathbb{C}^{*}\right)^{\emptyset}=\{1\}$. Note that $\left(\mathbb{C}^{*}\right)^{\mathrm{W}}=V\left(\xi_{\mathrm{W}}\right)$, where $\xi \mathrm{W}$ is the sublattice of $\mathbb{Z}^{n}$ spanned by the basis vectors $\left\{e_{i} \mid i \notin \mathrm{W}\right\}$. From [12], we have the following description of the characteristic varieties of our toric complex:

$$
V^{i}\left(T_{L}\right)=\bigcup_{\mathrm{W}} V\left(\xi_{\mathrm{W}}\right)
$$

where the union is taken over all subsets $\mathrm{W} \subseteq \mathrm{V}$ for which there is a simplex $\sigma \in L_{\mathrm{V} \backslash \mathrm{W}}$ and an index $j \leq i$ such that $\tilde{H}_{j-1-|\sigma|}\left(\mathrm{lk}_{L_{\mathrm{W}}}(\sigma), \mathbb{C}\right) \neq 0$. Here, $L_{\mathrm{W}}$ denotes the subcomplex induced by $L$ on W, and $\operatorname{lk}_{K}(\sigma)$ denotes the link of a simplex $\sigma \in L$ in a subcomplex $K \subseteq L$.

From the above, we see that the assumptions from Corollary 13.3 are true for the classifying space of a right-angled Artin group, and, in fact, for any toric complex. Hence, we obtain the following corollaries. 
Corollary 13.4. Let $T_{L}$ be a toric complex. Then,

$$
\Omega_{A}^{i}\left(T_{L}\right)=\Gamma(H, A) \backslash \bigcup_{\mathrm{W}} q^{-1}\left(\sigma_{\bar{A}}(\xi \mathrm{W})\right)
$$

where each $\sigma_{\bar{A}}\left(\xi_{\mathrm{W}}\right) \subseteq \mathrm{Gr}_{n-r}\left(\mathbb{Q}^{n}\right)$ is the special Schubert variety corresponding to the coordinate plane $\xi \mathrm{W} \otimes \mathbb{Q}$, and the union is taken over all subsets $\mathrm{W} \subseteq \mathrm{V}$ for which there is a simplex $\sigma \in L_{\mathrm{V} \backslash \mathrm{W}}$ and an index $j \leq i$ such that $\tilde{H}_{j-1-|\sigma|}\left(\mathrm{k}_{L_{\mathrm{W}}}(\sigma), \mathbb{C}\right) \neq 0$.

Corollary 13.5. Let $T_{L}$ be a toric complex. If $T_{L}^{\bar{\nu}}$ is a free Abelian cover with finite Betti numbers up to some degree $i$, then all regular, finite Abelian covers of $T_{L}^{\bar{v}}$ also have finite Betti numbers up to degree $i$.

\subsection{When the translation order is coprime to $|\operatorname{Tors}(A)|$}

We now return to the general situation, where the characteristic variety is a union of torsion-translated subgroups. In the next proposition, we identify a condition on the order of translation of these subgroups, insuring that diagram (4.7) is a pull-back diagram.

As usual, let $A$ be a quotient of $H=H_{1}(X, \mathbb{Z})$, and let $\bar{A}=A / \operatorname{Tors}(A)$ be its maximal torsion-free quotient. Recall that the canonical projection, $q: \Gamma(H, A) \rightarrow$ $\Gamma(H, \bar{A})$, restricts to a map $\left.q\right|_{\Omega_{A}^{i}(X)}: \Omega_{A}^{i}(X) \rightarrow \Omega_{\bar{A}}^{i}(X)$, and that resulting commuting square is a pull-back diagram if and only if $\Omega_{A}^{i}(X)$ is the full pre-image of $q$.

Using Proposition 8.13 and Theorem 10.5, we obtain the following consequence:

Proposition 13.6. Suppose the characteristic variety $V^{i}(X)$ is of the form $\bigcup_{j} \rho_{j} T_{j}$, where each $T_{j} \subset \widehat{H}$ is an algebraic subgroup, and each $\overline{\rho_{j}} \in \widehat{H} / T_{j}$ has finite order, coprime to the order of $\operatorname{Tors}(A)$. Then $\Omega_{A}^{i}(X)=q^{-1}\left(\Omega_{\bar{A}}^{i}(X)\right)$.

Here is an application. As usual, let $X$ be a connected CW-complex with finite $k$-skeleton. Assume $H=H_{1}(X, \mathbb{Z})$ has no torsion, and identify the character torus $\widehat{H}$ with $\left(\mathbb{C}^{*}\right)^{n}$, where $n=b_{1}(X)$.

Corollary 13.7. Suppose that, for some $i \leq k$, there is an $(n-1)$-dimensional subspace $L \subseteq H^{1}(X ; \mathbb{Q})$ such that $V^{i}(X)=\left(\bigcup_{\alpha} \rho_{\alpha} T\right) \cup Z$, where $Z$ is a finite set, $T=\exp (L \otimes \mathbb{C})$ and $\rho_{\alpha}$ is a torsion element in $\left(\mathbb{C}^{*}\right)^{n}$ of order coprime to that of $\operatorname{Tors}(A)$, for each $\alpha$. Let $r=\operatorname{rank} A$. Then,

$$
\Omega_{A}^{i}(X)= \begin{cases}\Gamma(H, A), & \text { if } r=1 ; \\ q^{-1}\left(\operatorname{Gr}_{r}(L)\right), & \text { if } 1<r<n ; \\ \emptyset, & \text { if } r \geq n .\end{cases}
$$


Proof. From [14, Proposition 8.6], we have

$$
\Omega_{r}^{i}(X)= \begin{cases}\mathbb{Q P}^{n-1} & \text { if } r=1 \\ \operatorname{Gr}_{r}(L) & \text { if } 1<r<n \\ \emptyset & \text { if } r \geq n .\end{cases}
$$

On the other hand, Proposition 13.6 shows that $\Omega_{A}^{i}(X)=q^{-1}\left(\Omega_{r}^{i}(X)\right)$, and so the desired conclusion follows.

\subsection{When the translation order divides $|\operatorname{Tors}(A)|$}

To conclude this section, we give some sufficient conditions on the groups $H$ and $A$, and on the order of translation of the subgroups comprising $V^{i}(X)$, insuring that diagram (4.7) is not a pull-back diagram.

Proposition 8.16 and Theorem 10.5 yield the following immediate application:

Corollary 13.8. Let $X$ be a connected $C W$-complex with finite $k$-skeleton. Assume that:

(i) The group $H=H_{1}(X, \mathbb{Z})$ is torsion-free, and $A$ is a quotient of $H$;

(ii) There is a degree $i \leq k$ such that the positive-dimensional components of $V^{i}(X)$ form a torsion-translated subgroup $\rho T$ inside $\widehat{H}$;

(iii) The rank of $A$ is less than the rank of $H$, and $\operatorname{ord}(\rho)$ divides $c(A)$.

Then $\Omega_{A}^{i}(X) \varsubsetneqq q^{-1}\left(\Omega_{\frac{i}{A}}(X)\right)$.

Note that the hypotheses of Corollary 13.8 are satisfied in Examples 12.5 and 12.7, thus explaining why, in both cases, diagram (4.7) is not a pull-back diagram. Here is one more situation when that happens:

Corollary 13.9. Suppose $V^{i}(X)=\rho_{1} T_{1} \cup \cdots \cup \rho_{s} T_{s}$, with each $T_{j}$ an algebraic subgroup of $\widehat{H}$ and each $\rho_{j}$ a torsion element in $\widehat{H} \backslash T_{j}$. Furthermore, suppose that:

(i) The identity component of $T_{1}$ is not contained in $T^{\prime}=T_{2} \cdots T_{s}$ (internal product in $\widehat{H}$ );

(ii) The order of $\rho_{1}$ divides $c(A)$;

(iii) $\operatorname{rank} A<\operatorname{rank} H-\operatorname{dim} T^{\prime}$.

Then $\Omega_{A}^{i}(X) \varsubsetneqq q^{-1}\left(\Omega_{\bar{A}}^{i}(X)\right)$.

Proof. Split $H$ as a direct sum, $H^{\prime} \oplus H^{\prime \prime}$, so that $\widehat{H}^{\prime}=T^{\prime}$ and $\widehat{H}^{\prime \prime}=\widehat{H} / T^{\prime}$. Let $p: H \rightarrow H^{\prime \prime}$ be the canonical projection, and let $\hat{p}: \widehat{H}^{\prime \prime} \rightarrow \widehat{H}$ be the induced morphism. By assumption (i), we have that $\hat{p}^{-1}\left(T_{1}\right)$ is a positive-dimensional algebraic subgroup of $\widehat{H}^{\prime \prime}$. Thus, the positive-dimensional components of $W=V^{i}(X) \cap \widehat{H}^{\prime \prime}$ 
form a torsion-translated subgroup of $\widehat{H}^{\prime \prime}$, namely, $\hat{p}^{-1}\left(\rho_{1} T_{1}\right)$. Moreover, assumptions (ii) and (iii) imply that $\operatorname{rank} A<\operatorname{rank} H^{\prime \prime}$ and $\operatorname{ord}\left(\hat{p}^{-1}\left(\rho_{1}\right)\right)$ divides $c(A)$.

Applying now Proposition 8.16 to the algebraic group $\widehat{H}^{\prime \prime}$ and to the subvariety $W$ yields an epimorphism $\mu: H^{\prime \prime} \rightarrow A$ such that $\operatorname{im}(\hat{\bar{\mu}}) \cap \hat{p}^{-1}\left(\rho_{1} T_{1}\right)$ is finite, and $\operatorname{im}(\hat{\mu}) \cap \hat{p}^{-1}\left(\rho_{1} T_{1}\right)$ is infinite. Setting $v=\mu \circ p$, we see that $\operatorname{im}(\hat{v})=\operatorname{im}(\hat{\mu})$. Thus, $\operatorname{im}(\hat{\bar{v}}) \cap V^{i}(X)$ is finite, while $\operatorname{im}(\hat{v}) \cap V^{i}(X)$ is infinite.

\section{Quasi-projective varieties}

We conclude with a discussion of the generalized Dwyer-Fried sets of smooth, quasi-projective varieties.

\subsection{Characteristic varieties}

A space $X$ is said to be a (smooth) quasi-projective variety if there is a smooth, complex projective variety $\bar{X}$ and a normal-crossings divisor $D$ such that $X=$ $\bar{X} \backslash D$. For instance, $X$ could be the complement of an algebraic hypersurface in $\mathbb{C P}^{d}$.

The structure of the characteristic varieties of such spaces was determined through the work of Beauville, Green and Lazarsfeld, Simpson, Campana, and Arapura in the 1990s, and further refined in recent years. We summarize these results, essentially in the form proved by Arapura.

Theorem 14.1 ([1]). Let $X=\bar{X} \backslash D$, where $\bar{X}$ is a smooth, projective variety and $D$ is a normal-crossings divisor:

(i) If either $D=\emptyset$ or $b_{1}(\bar{X})=0$, then each characteristic variety $V^{i}(X)$ is a finite union of unitary translates of algebraic subtori of $T=H^{1}\left(X, \mathbb{C}^{*}\right)$;

(ii) In degree $i=1$, the condition that $b_{1}(\bar{X})=0$ if $D \neq \varnothing$ may be lifted. Furthermore, each positive-dimensional component of $V^{1}(X)$ is of the form $\rho \cdot S$, where $S$ is an algebraic subtorus of $T$, and $\rho$ is a torsion character.

For instance, if $C$ is a connected, smooth complex curve of negative Euler characteristic, then $V^{1}(C)$ is the full character group $H^{1}\left(C, \mathbb{C}^{*}\right)$. For an arbitrary smooth, quasi-projective variety $X$, each positive-dimensional component of $V^{1}(X)$ arises by pullback along a suitable orbifold fibration (or, pencil). More precisely, if $\rho \cdot S$ is such a component, then $S=f^{*}\left(H^{1}\left(C, \mathbb{C}^{*}\right)\right)$, for some curve $C$, and some holomorphic, surjective map $f: X \rightarrow C$ with connected generic fiber.

Using this interpretation, together with recent work of Dimca, Artal-Bartolo, Cogolludo, and Matei (as recounted in [14]), we can describe the variety $V^{1}(X)$, as follows.

Theorem 14.2. Let $X$ be a smooth, quasi-projective variety. Then

$$
V^{1}(X)=\bigcup_{\xi \in \Lambda} V(\xi) \cup \bigcup_{\xi \in \Lambda^{\prime}}(V(\xi) \backslash V(\bar{\xi})) \cup Z,
$$


where $Z$ is a finite subset of $T=H^{1}\left(X, \mathbb{C}^{*}\right)$, and $\Lambda$ and $\Lambda^{\prime}$ are certain (finite) collections of subgroups of $H=H_{1}(X, \mathbb{Z})$.

\subsection{Dwyer-Fried sets}

Using now Theorem 14.2 (and keeping the notation therein), Proposition 8.12 yields the following structural result for the degree 1 Dwyer-Fried sets of a smooth, quasiprojective variety.

Theorem 14.3. Let $A$ be a quotient of $H$. Then

$$
\Omega_{A}^{1}(X)=\Gamma(H, A) \backslash\left(\bigcup_{\xi \in \Lambda} q^{-1}\left(\sigma_{\bar{A}}(\bar{\xi})\right) \cup \bigcup_{\xi \in \Lambda^{\prime}}\left(q^{-1}\left(\sigma_{\bar{A}}(\bar{\xi})\right) \cap \theta_{A}(\xi)\right)\right) .
$$

In certain situations, more can be said. For instance, Proposition 13.6 yields the following corollary.

Corollary 14.4. Suppose the order of $\bar{\xi} / \xi$ is coprime to $c(A)$, for each $\xi \in \Lambda^{\prime}$. Then $\Omega_{A}^{1}(X)=q^{-1}\left(\Omega_{A}^{1}(X)\right)$.

Similarly, Corollary 13.9 has the following consequence:

Corollary 14.5. Suppose $H$ is torsion-free, and there is a subgroup $\chi \in \Lambda^{\prime}$ such that:

(i) $V(\bar{\chi})$ is not contained in $T^{\prime}:=V\left(\bigcap_{\xi \in \Lambda \cup \Lambda^{\prime} \backslash\{\chi\}} \xi\right)$;

(ii) There is a non-zero element in $\bar{\chi} / \chi$ whose order divides $c(A)$;

(iii) $\operatorname{rank} A<\operatorname{codim} T^{\prime}$.

Then $\Omega_{A}^{1}(X) \varsubsetneqq q^{-1}\left(\Omega \frac{1}{A}(X)\right)$.

For the remainder of this section, we shall give some concrete examples of quasi-projective manifolds $X$ for which the computation of the sets $\Omega_{A}^{1}(X)$ can be carried out explicitly.

\subsection{Brieskorn manifolds}

Let $\left(a_{1}, \ldots, a_{n}\right)$ be an $n$-tuple of integers, with $a_{j} \geq 2$. Consider the variety $X$ in $\mathbb{C}^{n}$ defined by the equations $c_{j 1} x_{1}^{a_{1}}+\cdots+c_{j n} x_{n}^{a_{n}}=0$, for $1 \leq j \leq n-2$. Assuming all maximal minors of the matrix $\left(c_{j k}\right)$ are non-zero, $X$ is a quasi-homogeneous surface, with an isolated singularity at 0 .

The space $X$ admits a good $\mathbb{C}^{*}$-action. Set $X^{*}=X \backslash 0$, and let $p: X^{*} \rightarrow C$ be the corresponding projection onto a smooth projective curve. Then $p^{*}: H^{1}(C, \mathbb{C}) \rightarrow$ $H^{1}\left(X^{*}, \mathbb{C}\right)$ is an isomorphism, the torsion subgroup of $H=H_{1}\left(X^{*}, \mathbb{Z}\right)$ coincides with the kernel of $p_{*}: H_{1}\left(X^{*}, \mathbb{Z}\right) \rightarrow H_{1}(C, \mathbb{Z})$. 
By definition, the Brieskorn manifold $M=\Sigma\left(a_{1}, \ldots, a_{n}\right)$ is the link of the quasi-homogenous singularity $(X, 0)$. As such, $M$ is a closed, smooth, oriented 3-manifold homotopy equivalent to $X^{*}$. Put

$$
l=\operatorname{lcm}\left(a_{1}, \ldots, a_{n}\right), \quad l_{j}=\operatorname{lcm}\left(a_{1}, \ldots, \widehat{a_{j}}, \ldots, a_{n}\right), \quad a=a_{1} \cdots a_{n} .
$$

The $S^{1}$-equivalent homeomorphism type of $M$ is determined by the following Seifert invariants associated to the projection $\left.p\right|_{M}: M \rightarrow C$ :

- The exceptional orbit data, $\left(s_{1}\left(\alpha_{1}, \beta_{1}\right), \cdots, s_{n}\left(\alpha_{n}, \beta_{n}\right)\right)$, with $\alpha_{j}=l / l_{j}, \beta_{j} l \equiv$ $a_{j} \bmod \alpha_{j}$ and $s_{j}=a /\left(a_{j} l_{j}\right)$, where $s_{j}=\left(\alpha_{j} \beta_{j}\right)$ means $\left(\alpha_{j} \beta_{j}\right)$ repeated $s_{j}$ times, unless $\alpha_{j}=1$, in which case $s_{j}=\left(\alpha_{j} \beta_{j}\right)$ is to be removed from the list;

- The genus of the base curve, given by $g=\frac{1}{2}\left(2+(n-2) a / l-\sum_{j=1}^{n} s_{j}\right)$;

- The (rational) Euler number of the Seifert fibration, given by $e=-a / l^{2}$.

The group $H=H_{1}(M, \mathbb{Z})$ has rank $2 g$, and torsion part of order $\alpha_{1}^{s_{1}} \cdots \alpha_{n}^{s_{n}} \cdot|e|$. Identify the character group $\widehat{H}$ with a disjoint union of copies of $\widehat{H}_{0}=\left(\mathbb{C}^{*}\right)^{2 g}$, indexed by Tors $(H)$, and set $\alpha=\alpha_{1}^{s_{1}} \cdots \alpha_{n}^{S_{n}} / \operatorname{lcm}\left(\alpha_{1}, \ldots, \alpha_{n}\right)$.

Proposition 14.6 ([5]). The positive-dimensional components of $V^{1}(M)$ are as follows:

(i) $\alpha-1$ translated copies of $\widehat{H}_{0}$, if $g=1$;

(ii) $\widehat{H}_{0}$, together with $\alpha-1$ translated copies of $\widehat{H}_{0}$, if $g>1$.

Denote the elements in Tors $(H)$ corresponding to the $\alpha-1$ translated copies of $\widehat{H}_{0}$ by $h_{1}, \ldots, h_{\alpha-1}$. We then have the following corollaries.

Corollary 14.7. Let $M=\Sigma\left(a_{1}, \ldots, a_{n}\right)$ be a Brieskorn manifold, and let $A$ be a quotient of $H=H_{1}(M, \mathbb{Z})$, with $r=\operatorname{rank} A$.

(i) If $g>1$, then $\Omega_{A}^{1}(M)=\Omega \frac{1}{A}=\emptyset$.

(ii) If $g=1$, then $\Omega \frac{1}{A}(M)=\operatorname{Gr}_{r}\left(\mathbb{Q}^{2 g}\right)$, while

$$
\Omega_{A}^{1}(M)=\left\{[v] \in \Gamma(H, A) \mid v\left(h_{i}\right)=0 \text { for } i=1, \ldots, \alpha-1\right\} .
$$

Corollary 14.8. Suppose $g=1$ and $\alpha>1$. Then $\Omega_{\bar{H}}^{1}(M)=\{p t\}$, yet $\Omega_{H}^{1}(M)=$ $\emptyset$; that is, $b_{1}\left(M^{\mathrm{fab}}\right)<\infty$, yet $b_{1}\left(M^{\mathrm{ab}}\right)=\infty$.

Example 14.9. Consider the Brieskorn manifold $M=\Sigma(2,4,8)$. Using the algorithm described by Milnor in [11], we see that the fundamental group of $M$ has presentation

$$
G=\left\langle x_{1}, x_{2}, x_{3} \mid x_{1} x_{3}^{2}=x_{3}^{2} x_{1}, x_{2} x_{3}^{2}=x_{3}^{2} x_{2}, x_{3}^{2}\left(x_{3} x_{1} x_{2} x_{1}^{-1} x_{2}^{-1}\right)^{2}=1\right\rangle,
$$


while its Abelianization is $H=\mathbb{Z}^{2} \oplus \mathbb{Z}_{4}$. Identifying $\widehat{H}=\left(\mathbb{C}^{*}\right)^{2} \times\{ \pm 1, \pm i\}$, we find that $V^{1}(M)=\{1\} \cup\left(\mathbb{C}^{*}\right)^{2} \times\{-1\}$. (The positive-dimensional component in $V^{1}(M)$ arises from an elliptic orbifold fibration $X \rightarrow \Sigma_{1}$ with two multiple fibers, each of multiplicity 2.) By Proposition 11.6, then, $b_{1}\left(M^{\text {fab }}\right)<\infty$, while $b_{1}\left(M^{\mathrm{ab}}\right)=\infty$.

Now take $A=\mathbb{Z} \oplus \mathbb{Z}_{4}$. Applying Corollary 14.7 (with $g=1$ and $\alpha=2$ ), we conclude that $\Omega_{\frac{1}{A}}(M)=\mathbb{Q P}^{1}$, while $\Omega_{A}^{1}(M)$ consists of two copies of $\Gamma\left(\mathbb{Z}^{2}, A\right)$, naturally embedded in $\Gamma(H, A)$.

\subsection{The Catanese-Ciliberto-Mendes Lopes surface}

We now give an example of a smooth, complex projective variety $M$ for which the generalized Dwyer-Fried sets exhibit the kind of subtle behavior predicted by Corollary 14.5.

Let $C_{1}$ be a (smooth, complex) curve of genus 2 with an elliptic involution $\sigma_{1}$ and $C_{2}$ a curve of genus 3 with a free involution $\sigma_{2}$. Then $\Sigma_{1}=C_{1} / \sigma_{1}$ is a curve of genus 1 , and $\Sigma_{2}=C_{2} / \sigma_{2}$ is a curve of genus 2 . The group $\mathbb{Z}_{2}$ acts freely on the product $C_{1} \times C_{2}$ via the involution $\sigma_{1} \times \sigma_{2}$; let $M$ be the quotient surface. This variety, whose construction goes back to Catanese, Ciliberto, and Mendes Lopes [3], is a minimal surface of general type with $p_{g}(M)=q(M)=3$ and $K_{M}^{2}=8$.

The projection $C_{1} \times C_{2} \rightarrow C_{1}$ descends to an orbifold fibration $f_{1}: M \rightarrow \Sigma_{1}$ with two multiple fibers, each of multiplicity 2 , while the projection $C_{1} \times C_{2} \rightarrow C_{2}$ descends to a holomorphic fibration $f_{2}: M \rightarrow \Sigma_{2}$. It is readily seen that $H=$ $H_{1}(M, \mathbb{Z})$ is isomorphic to $\mathbb{Z}^{6}$; fix a basis $e_{1}, \ldots, e_{6}$ for this group. A computation detailed in [14] shows that the characteristic variety $V^{1}(M) \subset\left(\mathbb{C}^{*}\right)^{6}$ has two components, corresponding to the above two pencils; more precisely,

$$
V^{1}(M)=V\left(\xi_{1}\right) \cup\left(V\left(\xi_{2}\right) \backslash V\left(\bar{\xi}_{2}\right)\right),
$$

where $\xi_{1}=\operatorname{span}\left\{e_{1}, e_{2}\right\}$ and $\xi_{2}=\operatorname{span}\left\{2 e_{3}, e_{4}, e_{5}, e_{6}\right\}$.

Now suppose $A$ is a quotient of $H=\mathbb{Z}^{6}$, and let $q: \Gamma(H, A) \rightarrow \Gamma(H, \bar{A})$ be the canonical projection. By Theorem 14.3,

$$
\Omega_{A}^{1}(M)=\Gamma(H, A) \backslash\left(q^{-1}\left(\sigma_{\bar{A}}\left(\xi_{1}\right)\right) \cup\left(q^{-1}\left(\sigma_{\bar{A}}\left(\bar{\xi}_{2}\right)\right) \cap \theta_{A}\left(\xi_{2}\right)\right)\right) .
$$

Let us describe explicitly this set in a concrete situation:

Example 14.10. Let $A=\mathbb{Z} \oplus \mathbb{Z}_{2}$, and identify $\bar{A}=\mathbb{Z}$ and $\Gamma(H, \mathbb{Z})=\mathbb{Q P}^{5}$. The fiber of $q$ is the set $\Gamma=\left(\mathbb{Z}_{2}^{5}\right)^{*}$. Given an epimorphism $v: H \rightarrow \mathbb{Z} \oplus \mathbb{Z}_{2}$, let $\bar{v}: H \rightarrow \mathbb{Z}$ and $v^{\prime}: H \rightarrow \mathbb{Z}_{2}$ be the composites of $v$ with the projections on the respective factors. The terms on the right-side of (14.2) are as follows:

- $\sigma_{\mathbb{Z}}\left(\xi_{1}\right)$ is the projective subspace $\mathbb{Q P}^{3}=\mathbb{P}\left(\left(H / \xi_{1}\right)^{\vee} \otimes \mathbb{Q}\right)$ spanned by $e_{3}, \ldots, e_{6}$;

- $\sigma_{\mathbb{Z}}\left(\bar{\xi}_{2}\right)$ is the projective line $\mathbb{Q P}^{1}=\mathbb{P}\left(\left(H / \xi_{2}\right)^{\vee} \otimes \mathbb{Q}\right)$ spanned by $e_{1}$ and $e_{2}$; 
- $q^{-1}\left(\mathbb{Q P}^{1}\right) \cap \theta_{A}\left(\xi_{2}\right)$ consists of those $[v]$ satisfying $\bar{v}\left(e_{3}\right)=\cdots=\bar{v}\left(e_{6}\right)=0$, and $v^{\prime}\left(e_{3}\right)=1, v^{\prime}\left(e_{4}\right)=v^{\prime}\left(e_{5}\right)=v^{\prime}\left(e_{6}\right)=0$.

This completes the description of the set $\Omega_{\mathbb{Z} \oplus \mathbb{Z}_{2}}^{1}(M)$. Clearly, $\Omega_{1}^{1}(M)=\mathbb{Q P}^{5} \backslash$ $\mathbb{Q P}^{3}$. Furthermore, note that the restriction map $q^{-1}\left(\mathbb{Q P}^{1}\right) \cap \theta_{A}\left(\xi_{2}\right) \rightarrow \mathbb{Q P}^{1}$ is a 2-to-1 surjection. Thus, the restriction map $\Omega_{\mathbb{Z} \oplus \mathbb{Z}_{2}}^{1}(M) \rightarrow \Omega_{1}^{1}(M)$ is not a set fibration: the fiber over $\Omega_{1}^{1}(M) \backslash \mathbb{Q P} \mathbb{P}^{1}$ has cardinality 31 , while the fiber over $\mathbb{Q P}^{1}$ has cardinality 29 .

\section{References}

[1] D. ARAPURA, Geometry of cohomology support loci for local systems, I, J. Algebraic Geom. 6 (1997), 563-597.

[2] M. ARTIN, "Algebra", Prentice Hall, Inc., Englewood Cliffs, NJ, 1991.

[3] F. Catanese, C. Ciliberto and M. Mendes Lopes, On the classification of irregular surfaces of general type with nonbirational bicanonical map, Trans. Amer. Math. Soc. 350 (1998), 275-308.

[4] A. DimCA, S. PAPAdima and A. I. SUCIU, Topology and geometry of cohomology jump loci, Duke Math. J. 148 (2009), 405-457.

[5] A. Dimca, S. PAPADima and A. SuCIU, Quasi-Kähler groups, 3-manifold groups, and formality, Math. Z. 268 (2011), 169-186.

[6] W. G. DWYER and D. FRIED, Homology of free abelian covers, I, Bull. London Math. Soc. 19 (1987), 350-352.

[7] R. H. Fox, Free differential calculus. I. Derivation in the free group ring, Ann. of Math. 57 (1953), 547-560.

[8] A. Hatcher, "Algebraic Topology", Cambridge University Press, Cambridge, 2002.

[9] E. HIRONAKA, Torsion points on an algebraic subset of an affine torus, Internat. Math. Res. Notices 1996 (1996), 953-982.

[10] D. MATEI and A. I. SUCIU, Hall invariants, homology of subgroups, and characteristic varieties, Int. Math. Res. Not. 2002 (2002), 465-503.

[11] J. MiLnOR, On the 3-dimensional Brieskorn manifolds $M(p, q, r)$, In: "Knots, Groups, and 3-manifolds", Ann. of Math. Studies, Vol. 84, Princeton Univ. Press, Princeton, NJ (1975), 175-225.

[12] S. PAPAdima and A. Suciu, Toric complexes and Artin kernels, Adv. Math. 220 (2009), 441-477.

[13] S. Papadima and A. Suciu, Bieri-Neumann-Strebel-Renz invariants and homology jumping loci, Proc. London Math. Soc. 100 (2010), 795-834.

[14] A. SuCIU, Characteristic varieties and Betti numbers of free abelian covers, Int. Math. Res. Not. (IMRN) (2014), 1063-1124.

[15] A. SUCIU, Y. YANG and G. ZHAO, Intersections of translated algebraic subtori, J. Pure Appl. Algebra 217 (2013), 481-494.

Department of Mathematics

Northeastern University

Boston, MA 02115, USA

a.suciu@neu.edu

yang.yap@husky.neu.edu

zhao.g@husky.neu.edu 